\title{
The histone deacetylase complex MiDAC regulates a neurodevelopmental gene expression program
}

Baisakhi Mondal, ${ }^{1}$ Hongjian Jin, ${ }^{2}$ Satish Kallappagoudar, ${ }^{1}$ Yurii Sedkov, ${ }^{1}$ Tanner Martinez, ${ }^{1}$ Monica F. Sentmanat, ${ }^{3}$ Chunliang Li, ${ }^{4}$ Yiping Fan, ${ }^{2}$ Shondra M. Pruett-Miller, ${ }^{1}$ Hans-Martin Herz $^{1, *}$

${ }^{1}$ Department of Cell \& Molecular Biology, St. Jude Children's Research Hospital, Memphis, TN 38105, USA.

${ }^{2}$ Department of Computational Biology, St. Jude Children's Research Hospital, Memphis, TN 38105, USA.

${ }^{3}$ Genome Engineering \& iPS Center, Department of Genetics, Washington University, St. Louis, MO 63110, USA.

${ }^{4}$ Department of Tumor Cell Biology, St. Jude Children's Research Hospital, Memphis, TN 38105, USA.

*Corresponding author. Tel. +1 901595 2058; E-mail: hans-martin.herz@stjude.org

\section{Running title}

MiDAC regulates neurodevelopmental genes

\section{Keywords}

Mitotic deacetylase complex MiDAC, DNTTIP1, ELMSAN1/MIDEAS, HDAC1, neurite outgrowth, SLIT and NETRIN signaling pathways, H4K20 acetylation 


\begin{abstract}
MiDAC is a recently identified histone deacetylase (HDAC) complex. While other HDAC complexes have been implicated in neurogenesis, the physiological role of MiDAC remains unknown. Here, we show that MiDAC constitutes an important regulator of neural differentiation. We demonstrate that MiDAC functions as a modulator of a neurodevelopmental gene expression program and binds to important regulators of neurite outgrowth. On the one hand, MiDAC upregulates gene expression by mediating the removal of H4K20ac on the promoters and enhancers of pro-neural genes such as those encoding the secreted ligands SLIT3 and NETRIN1 (NTN1). Conversely, MiDAC inhibits gene expression by reducing H3K27ac on promoterproximal and -distal elements of negative regulators of neurogenesis. Furthermore, loss of MiDAC results in neurite outgrowth defects that can be rescued by supplementation with SLIT3 and/or NTN1. These findings indicate a crucial role for MiDAC in regulating the ligands of the SLIT3 and NTN1 signaling axes to ensure the proper integrity of neurite development.
\end{abstract}




\section{Introduction}

Epigenetic regulators often constitute chromatin-modifying enzymes which catalyze the addition and removal of posttranslational modifications on histones and are involved in the control of gene expression by modulating chromatin structure and function. Among them, the families of histone acetyltransferases (HATs) and histone deacetylases (HDACs) acetylate and deacetylate lysine residues on histones and other proteins, respectively (Shahbazian \& Grunstein, 2007). Based on initial studies, a model emerged in which nuclear HDACs were thought to be recruited by transcription factors to facilitate transcriptional repression by creating a more condensed chromatin landscape at their target genes (Kadosh \& Struhl, 1998, Rundlett, Carmen et al., 1998, Yang, Inouye et al., 1996, Yang, Yao et al., 1997). However, more recently it was shown that HDACs tend to localize to transcriptionally active loci including promoters, gene bodies and enhancers (Wang, Zang et al., 2009). Additionally, gene expression profiling studies also demonstrated that knockout of individual HDACs resulted not only in upregulation but also downregulation of a significant number of genes suggesting both activating and repressive roles for HDACs in regulating gene transcription (Bernstein, Tong et al., 2000, Harrison, Georgiou et al., 2011, Yamaguchi, Cubizolles et al., 2010, Zupkovitz, Tischler et al., 2006).

HDAC1 and HDAC2 belong to the family of class I HDACs, are highly similar (83\% identity) and are involved in the control of gene expression by modulating chromatin structure and function. Their vital and often redundant roles in neurogenesis are well established (Chen, Wang et al., 2011, Graff, Rei et al., 2012, Guan, Haggarty et al., 2009, Jacob, Christen et al., 2011, Kim, Shen et al., 2010, Montgomery, Hsieh et al., 2009, Ye, Chen et al., 2009). They form the catalytic core of the SIN3, NuRD, CoREST and mitotic deacetylase (MiDAC) complexes (Kelly \& Cowley, 2013, Millard, Watson et al., 2017). HDAC1/2 activity and targeting to specific gene loci strongly depends on their incorporation into these complexes (Kelly \& Cowley, 2013, Millard et al., 2017). At least one of the scaffolding proteins within the NuRD, CoREST and MiDAC complexes contains an ELM2-SANT domain which is instrumental in recruiting and activating HDAC1/2 (Millard et al., 2017). While the molecular function of the SIN3, NuRD and CoREST complexes have been studied in greater detail little is known about the molecular function of MiDAC (Kelly \& Cowley, 2013, Millard et al., 2017). 
MiDAC is conserved from nematodes to humans (Bantscheff, Hopf et al., 2011, Hao, Xu et al., 2011). In humans, MiDAC is composed of HDAC1/2, the scaffolding protein DNTTIP1, and the ELM2-SANT domain containing scaffolding protein ELMSAN1 (also known as MIDEAS) and/or the closely ELMSAN1-related proteins TRERF1 and ZNF541 (ZFP541 or SHIP in mice) (Figure 1A) (Banks, Miah et al., 2018, Bantscheff et al., 2011, Choi, Han et al., 2008, Hao et al., 2011, Joshi, Greco et al., 2013). MiDAC constitutes a stoichiometric tetrameric complex that requires the N-terminal dimerization domain of DNTTIP1 for assembly. The C terminus of DNTTIP1 mediates MiDAC recruitment to nucleosomes in vitro (Itoh, Fairall et al., 2015). MiDAC specifically associates with cyclin A2 (CCNA2) and cell cycle dependent kinase 2 and interacts more prominently with certain HDAC inhibitors in mitotically arrested versus non-synchronized proliferating cells, suggesting a role in cell cycle regulation (Bantscheff et al., 2011, Hein, Hubner et al., 2015, Huttlin, Ting et al., 2015, Pagliuca, Collins et al., 2011). Before MiDAC was described as a complex its individual subunits DNTTIP1 and TRERF1 were reported to function predominantly as transcriptional activators for select genes of the steroidogenesis and ossification pathways, respectively (Gizard, Lavallee et al., 2001, Gizard, Lavallee et al., 2002, Gizard, Robillard et al., 2005, Gizard, Robillard et al., 2006, Gizard, Teissier et al., 2004, Koiwai, Kubota et al., 2015, Kubota, Koiwai et al., 2013). In agreement with these findings, ELMSAN1 was found to be associated with chromatin enriched for the histone mark H3K27ac suggesting a role in active transcription (Ji, Dadon et al., 2015). While SIN3, NuRD, and CoREST have been functionally characterized as regulators of neurogenesis, the physiological role of MiDAC remains unexplored (Andres, Burger et al., 1999, Knock, Pereira et al., 2015, Nitarska, Smith et al., 2016, Wang, Wu et al., 2016).

To decipher the physiological function of MiDAC, we employed mESCs as an experimental model system. We found that MiDAC is recruited to promoters and enhancers genome-wide, directly regulates a set of neurodevelopmental genes, and can act as both an activator and repressor of different gene sets to modulate gene expression by negatively regulating the repressive histone mark H4K20ac or the active histone mark H3K27ac, respectively. Specifically, MiDAC binds to promoters and enhancers of axon guidance ligands of the SLIT and NETRIN families and is required for their activation. Genetic deletion of MiDAC components results in inactivation of SLIT3 and NTN1 signaling during neural differentiation and severely impairs neurite outgrowth and network formation. These findings reveal a novel function for MiDAC in regulating neural 
bioRxiv preprint doi: https://doi.org/10.1101/2020.04.07.029751; this version posted April 7, 2020. The copyright holder for this preprint (which was not certified by peer review) is the author/funder, who has granted bioRxiv a license to display the preprint in perpetuity. It is made available under aCC-BY 4.0 International license.

gene expression programs to ensure proper neuronal maturation and/or neurite outgrowth during neurogenesis. 


\section{Results}

\section{DNTTIP1 interacts with ELMSAN1 and the histone deacetylase HDAC1 to form MiDAC in mESCs}

We began by investigating the function of DNTTIP1 and ELMSAN1, two scaffolding components of MiDAC, in mESCs (Figure 1A). Firstly, we generated and validated CRISPR/Cas9-mediated knock outs (KOs) of Dnttipl and Elmsanl in mESCs (Figure 1B; Figure S1A-F; Table S1). Indels in the identified Dnttipl KO and Elmsan1 KO clones resulted in the introduction of a premature stop codon downstream, producing KO clones with a complete loss of DNTTIP1 or ELMSAN1 protein compared to wild-type (WT) mESCs, respectively (Figure 1B; Figure S1A, B, F). To determine whether DNTTIP1 and ELMSAN1 function within MiDAC in mESCs, we examined the mRNA and protein levels of other MiDAC components in Dnttipl KO and Elmsanl KO mESCs (Bantscheff et al., 2011, Choi et al., 2008, Hao et al., 2011). Elmsan1 and Hdacl mRNA levels were unchanged, Hdac2 mRNA levels slightly reduced and Dnttip1 mRNA levels more strongly reduced in Dnttipl KO versus WT mESCs (Figure S1C, E). However, ELMSAN1 protein levels were severely reduced with no significant effects on HDAC1 or HDAC2 protein levels in Dnttipl KO versus WT mESCs (Figure 1B). Compared to WT mESCs, Elmsan1 KO mESCs showed no significant differences in Elmsan1, Dnttip1, Hdac1, or Hdac2 mRNA levels (Figure S1D, F); at the protein level, HDAC1 and HDAC2 appeared unaltered, but DNTTIP1 levels were significantly lower (Figure S1F). These results suggest that in mESCs MiDAC becomes destabilized upon loss of its core subunits, DNTTIP1 or ELMSAN1. Immunoprecipitation (IP) of ELMSAN1 in WT and Dnttipl KO mESCs confirmed the existence of an HDAC1-containing MiDAC complex (Figure 1C). Furthermore, consistent with the ELMSAN1 IP in WT and Dnttip1 KO mESCs, IP of DNTTIP1 in WT and Elmsan1 KO mESCs corroborated the presence of an HDAC1-containing MiDAC complex in mESCs (Figure S1G). However, we did not observe an interaction between ELMSAN1 and HDAC2 in Dnttip1 KO mESCs and DNTTIP1 and HDAC2 in Elmsan1 KO mESCs, suggesting that DNTTIP1, ELMSAN1 and HDAC1 interact to form MiDAC in mESCs (Figure 1C; Figure S1G).

\section{MiDAC controls a neurodevelopmental gene expression program}


To determine transcriptional targets of MiDAC, we performed RNA sequencing (RNA-seq) in WT, Dnttipl KO and Elmsan1 KO mESCs. We identified 493 downregulated and 368 upregulated genes in Dnttipl KO versus WT mESCs (Figure S2A; Table S2). Differential gene expression analysis via RNA-seq revealed 467 downregulated and 306 upregulated genes in Elmsan1 KO versus WT mESCs (Figure S2B; Table S2). The gene expression patterns between Dnttip1 KO and Elmsan $1 \mathrm{KO}$ mESCs were highly correlated $\left(\mathrm{R}^{2}=0.88\right)$ with many differentially expressed genes (DEGs) shared between Dnttipl KO and Elmsan1 KO mESCs (Figure 1D; Table S2). Of the DEGs in Dnttipl KO and Elmsanl KO mESCs a common set of 114 upregulated and 224 downregulated genes was identified (Figure 1E; Figure S2C). Pathway analysis of this MiDACregulated gene set revealed an enrichment for axon guidance signaling and neural development related genes among the downregulated category (Figure 1F). Similar results were also obtained when DEGs in only Dnttipl KO or only Elmsan1 KO mESCs were analyzed independently of each other (Figure S2D-G). Downregulated gene classes were generally associated with promoting neural differentiation (e.g., nervous system, axon guidance and GABAergic neurogenesis), whereas upregulated genes showed enrichment for pathways associated with repression of neuronal development (e.g., antagonists of nerve growth factor (NGF) signaling) (Figure S2D-G; Table S2). Importantly, we identified a set of neurodevelopmental genes that is associated with neurogenesis, axon guidance, and neurotransmitter receptor signaling and coregulated by both ELMSAN1 and DNTTIP1 (Figure 1D, G). However, loss of MiDAC function did not affect self-renewal or pluripotency as evaluated by mRNA levels of the pluripotency factors Sox2, Pou5f1, and Nanog (Figure S3A, B), nor did it alter the percentage of alkaline phosphatase (AP)-positive colonies (Figure S3C). Furthermore, DNTTIP1 or ELMSAN1 loss in mESCs did not alter the proliferation rate or cell cycle profile (Figure S3D, E), in contrast to the reported growth-promoting function of DNTTIP1 in oral and non-small cell lung cancer (Sawai, Kasamatsu et al., 2018, Zhang, Wang et al., 2018). Gene expression of Ccna2 and the cell cycle inhibitors Cdkn1a (p21) and Cdkn1b (p27) whose protein products were reported to interact with and/or be regulated by DNTTIP1 were also unaffected in Dnttipl KO and Elmsan1 KO mESCs (Figure S3F) (Hein et al., 2015, Huttlin et al., 2015, Pagliuca et al., 2011, Sawai et al., 2018). Together, this suggests that loss of MiDAC function does not impair self-renewal, pluripotency or the cell cycle distribution in mESCs. Rather, our findings indicate that DNTTIP1 and ELMSAN1 regulate a highly similar set of target genes in mESCs and that MiDAC controls a neurodevelopmental gene 
expression program, including genes that are important regulators of neurite outgrowth and morphogenesis.

\section{MiDAC regulates neurite outgrowth}

To investigate the function of MiDAC in neuronal development, we differentiated mESCs into neuro-ectoderm (NE) (Figure 2A) (Li, Yu et al., 2009). We evaluated WT, Dnttipl KO and Elmsan1 KO NE after 8 days of differentiation, a time point that corresponds to the initiation of differentiation into NPCs, which is accompanied by increased expression of nestin (Nes) and Pax6 as well as increased protein levels of the early NE differentiation markers, PAX6 and MASH1. No significant differences in mRNA and protein levels of the above-mentioned NPC markers were detected between WT and Dnttipl KO or Elmsan1 KO cells at day 0 or day 8 (Figure S4A-D). Moreover, PAX6-positive cells from WT, Dnttip1 KO and Elmsan1 KO NE at day 8 displayed a similar cell cycle profile, indicating no differences in cell cycle distribution in NPCs (Figure S4E). These results demonstrate that loss of MiDAC function in mESCs does not affect the initiation of differentiation into the NE lineage. Upon extended differentiation through day 12, many WT cells underwent extensive morphological changes and developed into mature neurons with long neurites while the neurite length of Dnttip1 KO and Elmsan1 KO neurons was substantially shorter (Figure 2B). Consistent with these observations, Dnttipl KO and Elmsanl KO neurons showed significantly reduced Tubb3 and Map2 mRNA levels (immature and mature neuron marker genes, respectively) compared to WT neurons, suggesting that loss of MiDAC function affects neuronal maturation and/or differentiation (Figure 2C, D). Dnttipl KO and Elmsanl KO neurons also showed a significant decrease in the average neurite length per neuron as well as the total number of neurites per neuron compared to WT neurons as assessed by MAP2 staining at day 12 of differentiation (Figure 2B, E, F). However, the percentage of neurons within the total differentiated cell population remained unchanged between WT and Dnttipl KO or Elmsan1 KO NE (Figure 2G; Figure S4F). We next performed qRT-PCR for a subset of NE genes involved in neurite outgrowth and morphogenesis at day 12. Similar to our findings in mESCs, Dnttip1 KO and Elmsan1 KO NE showed decreased expression of several positive regulators of neuronal development and axon guidance (e.g., Slit3, Ntn1, Ncam1) as well as increased expression of repressors of neuronal development (e.g., Spry4, Id1, Pacsin1) compared to WT neurons (Figure 2H). Overall, our data suggest that MiDAC is required to transcriptionally control a gene set of 
regulators that are important for neuronal maturation or neurite outgrowth but that loss of MiDAC function does not affect initiation of neuronal differentiation per se.

\section{MiDAC binds to and modulates the expression of genes that regulate neural differentiation}

\section{and neurite outgrowth}

To better understand how MiDAC regulates gene expression in mESCs, we performed ChIP sequencing (ChIP-seq) in WT, Dnttipl KO and Elmsan1 KO mESCs to map the genome-wide occupancy of DNTTIP1. This analysis identified 61,505 DNTTIP1-specific peaks, of which 22\% localized within $+/-1 \mathrm{~kb}$ of a transcription start site (TSS) (Figure 3A; Table S3). The remaining $78 \%$ of DNTTIP1 binding sites were located upstream of (24\%), downstream of (12\%), or within (43\%) a gene. Of the 47,657 non-TSS DNTTIP1 peaks, a majority (84\%) was found at a distance of $>5 \mathrm{~kb}$ from the TSS (Figure 3A; Table S3). Interestingly, a significant number of DNTTIP1 binding sites overlapped with the active histone marks H3K4me1 and/or H3K27ac and TSSassociated DNTTIP1 peaks were strongly enriched for the active histone mark H3K4me3 along with H3K27ac and H3K4me1 (Figure 3B; Figure S5A). Furthermore, low DNTTIP1 binding at TSS correlated with increased enrichment of the repressive histone mark H3K27me3 (Figure S5A). Non-TSS DNTTIP1 binding sites were enriched for the enhancer marks H3K4me1 and H3K27ac, hinting at a potential role for DNTTIP1 in enhancer-mediated processes (Figure S5A). We next correlated our DNTTIP1 ChIP-seq data with gene expression data from Dnttipl KO mESCs. This analysis revealed that out of 493 downregulated genes in Dnttip1 KO mESCs, 358 genes (73\%) were bound by DNTTIP1, while out of 368 upregulated genes in Dnttip1 KO mESCs, 314 genes $(85 \%)$ were occupied by DNTTIP1 at either their promoters or other gene regulatory regions such as enhancers (Figure 3C; Figure S5B, C; Table S4). Importantly, an even higher proportion of differentially expressed neurodevelopmental genes was also bound by DNTTIP1 (36 out of $39(92 \%)$ in the downregulated and 22 out of $23(96 \%)$ in the upregulated category) (Table S4). This overlap is highly significant and indicates that the majority of DEGs in Dnttipl KO mESCs constitute direct targets of DNTTIP1, including important regulators of neuronal differentiation and neurite outgrowth. Moreover, motif analysis of DNTTIP1 peaks revealed that DNTTIP1 binding sites associated with down- or upregulated genes in Dnttipl KO mESCs are selectively enriched for transcription factor (TF) binding motifs, including some that have been implicated in neurogenesis (Figure S5D). These TFs include RBFOX2 (associated with genes 
upregulated in Dnttipl KO mESCs) and ELK1, a member of the ETS TF family (associated with genes downregulated in Dnttipl KO mESCs) (Figure S5D) (Besnard, Galan-Rodriguez et al., 2011, Gehman, Meera et al., 2012). We further assessed the relationship between DNTTIP1 and ELMSAN1 by determining the genome-wide occupancy pattern of DNTTIP1 in Elmsan1 KO mESCs. While no DNTTIP1 binding to chromatin could be detected in Dnttipl KO mESCs, DNTTIP1 association with chromatin was strongly reduced in the absence of ELMSAN1 (Figure 3D). However, some DNTTIP1 enrichment in Elmsan1 KO mESCs could still be observed, suggesting that DNTTIP1 retains the ability to bind to chromatin when ELMSAN1 is depleted (Figure 3D). To study the genome-wide MiDAC binding landscape in mESCs, we compared the occupancy patterns of DNTTIP1 and HDAC1. We found that many DNTTIP1-bound regions (58\%) also displayed considerable enrichment for HDAC1, and that $40 \%$ of all HDAC1 peaks colocalized with DNTTIP1 (Figure 3E). Considering that HDAC1 also exists within several other complexes, including SIN3, NuRD, and CoREST, this finding suggests that a significant number of HDAC1-occupied loci is bound by MiDAC. Collectively, our findings suggest that MiDAC localizes genome-wide to enhancers and promoters in mESCs, thereby transcriptionally activating or inhibiting neurodevelopmental genes. We next examined activators and repressors of neurite outgrowth more specifically. We investigated SLIT3 and NETRIN1 (NTN1), which are key ligands of the SLIT/ROBO and NETRIN/DCC/UNC signaling pathways, and are regulators of neuron maturation and neurite outgrowth but are also playing roles in angiogenesis, lung morphogenesis, mammary gland development and cancer progression involving processes such as cell migration, cell interaction and cell adhesion (Bashaw \& Klein, 2010, Blockus \& Chedotal, 2016, Lai Wing Sun, Correia et al., 2011, Seiradake, Jones et al., 2016). Both Slit3 and Ntn1 mRNA levels were decreased in Dnttipl KO and Elmsan1 KO versus WT mESCs and NE (Figure 1G, 2H; Table S2). Furthermore, DNTTIP1 was directly bound to the promoters and putative intraand intergenic enhancers of Slit3 and Ntn1 in mESCs and many of these gene regulatory elements were also co-bound by HDAC1 (Figure 3F; Figure S5E). Conversely, a repressor of neurite outgrowth and morphogenesis, sprouty4 (Spry4), and an inhibitor of neural differentiation, Idl, were both transcriptionally upregulated in mESCs and NE upon loss of DNTTIP1 or ELMSAN1 (Figure 1G, 2H; Table S2) (Alsina, Irala et al., 2012, Lyden, Young et al., 1999, Nam \& Benezra, 2009). DNTTIP1 and HDAC1 co-occupied the promoters and putative enhancers of Spry4 and IdI (Figure 3G; Figure S5F). In summary, these results indicate that MiDAC binds to regulatory 
regions of activators and repressors of neural differentiation and neurite outgrowth, thereby positively or negatively modulating their transcriptional output, which is in accordance with the phenotypic defects observed in Dnttipl KO and Elmsan1 KO neurons (Figure 2B, E, F). We next sought to identify potential histone substrates of MiDAC by screening for global changes of several histone acetylation marks by performing western blots in WT, Dnttip1 KO, and Elmsan1 KO mESCs. Of the histone acetylation marks tested, only H4K20ac was found to be consistently increased in Dnttipl KO and Elmsan1 KO mESCs, suggesting that H4K20ac could be a major MiDAC substrate (Fig. 3H). Interestingly, H4K20ac has been recently described as a repressive histone acetylation mark, and its targeting by MiDAC might explain why we observed a greater number of downregulated compared to upregulated genes in Dnttipl KO and Elmsan1 KO mESCs versus WT mESCs (Kaimori, Maehara et al., 2016).

\section{MiDAC directly targets positive and negative regulators of neurite outgrowth during neural differentiation}

To further decipher the role of MiDAC in regulating select target genes during neurogenesis, we performed ChIP analysis of DNTTIP1, ELMSAN1, HDAC1 and the histone acetylation marks $\mathrm{H} 3 \mathrm{~K} 27 \mathrm{ac}$ (active) and H4K20ac (repressive) in NE after 12 days of differentiation. We focused our attention on the four previously described neurodevelopmental genes-Slit3, Ntn1, Spry4, and Id 1 - that we had identified as being bound by MiDAC in mESCs and transcriptionally regulated in mESCs and NE amongst other target genes (Figure 1G, 2H, 3F, G; Figure S5E, F). In WT NE, DNTTIP1, ELMSAN1, and HDAC1 occupied the promoter regions and putative enhancer regions of all four genes, and MiDAC binding was abrogated in Dnttipl KO and Elmsan1 KO NE (Figure 4A-C, F-H; Figure S6A-C, F-H). Interestingly, we observed gene-specific changes in the active histone mark $\mathrm{H} 3 \mathrm{~K} 27 \mathrm{ac}$ and the repressive histone mark H4K20ac depending on whether a gene was upregulated or downregulated in Dnttipl KO and Elmsan1 KO compared to WT mESCs and NE. For example, genes that were positively regulated by MiDAC in NE, such as Slit3 and Ntn1, displayed a strong increase in H4K20ac in Dnttipl KO and Elmsan 1 KO versus WT NE on promoter and associated enhancer regions without major changes in H3K27ac (Figure 4D, E; Figure S6D, E). The reverse scenario was observed for genes that were repressed by MiDAC in NE cells, such as Spry4 and Id1, which showed a higher enrichment of H3K27ac at their promoter and putative enhancer regions without any alteration in H4K20ac in Dnttip1 KO and Elmsan1 KO 
compared to WT NE (Figure 4I, J; Figure S6I, J). In summary, our data indicate that MiDAC differentially regulates the active $\mathrm{H} 3 \mathrm{~K} 27 \mathrm{ac}$ and repressive H4K20ac marks on neurodevelopmental genes during neurogenesis. Furthermore, genes that are positively regulated by MiDAC tend to be activators of neurite outgrowth and morphogenesis, whereas genes that are under negative control of MiDAC appear to be repressors of neural differentiation.

\section{MiDAC regulates neurite outgrowth via the SLIT3/ROBO3 and NTN1/UNC5B signaling} pathways

We noticed that several downregulated genes in Dnttipl KO and Elmsan1 KO mESCs and NE encoded secreted ligands of the SLIT and NETRIN families, which are important regulators of neurite development and axon guidance signaling (Figure 1G, 2H). Furthermore, our findings also indicated that MiDAC is bound to promoters and enhancers of Slit3 and Ntn1, suggesting that MiDAC directly activates the genes encoding these secreted ligands during neurogenesis (Figure 3F, G; Figure S5E, F). However, MiDAC did not transcriptionally regulate the genes of their cognate receptors Robo3 and Unc5b in mESCs and NE and even though it was bound to the promoter and putative enhancer regions of $U n c 5 b$ in mESCs no enrichment for MiDAC components was detected on the promoters of Robo3 and Unc5b in NE (Figure S7A, B-F). Thus, we hypothesized that the observed defect in neurite outgrowth upon loss of MiDAC might be caused at least in part by transcriptional downregulation of Slit3 and/or Ntn1. To address this question, we differentiated WT, Dnttipl KO and Elmsan1 KO mESCs into NE for 12 days and supplemented Dnttip1 KO and Elmsan1 KO cells daily with conditioned medium (CM) from differentiating WT cells from day 7 onward (Figure S8A). After 12 days of differentiation, NE was stained for MAP2 followed by analysis of neurite length (Figure 5A). Consistent with our previous findings, the average neurite length per neuron was reduced in Dnttipl KO and Elmsan1 KO compared to WT neurons and was partly restored upon treatment with CM of WT NE (Figure $\mathbf{5 A}, \mathbf{B})$. Interestingly, classification of the neurite length into two categories, $<50 \mu \mathrm{m}$ or $\geq 50 \mu \mathrm{m}$, showed that only longer neurites ( $\geq 50 \mu \mathrm{m}$ in length) were significantly affected in Dnttipl KO and Elmsan1 KO neurons and could be selectively rescued by supplementation with WT CM (Figure 5B). In addition, the reduced number of neurites per neuron in Dnttipl KO and Elmsan1 KO versus WT neurons was also significantly restored upon treatment with CM of WT NE (Figure 5C). To further validate these findings, we used a chamber assay to co-culture granule neuron progenitor 
cells (GNPs) in the lower chamber in parallel with differentiating WT, Dnttip1 KO or Elmsan1 $\mathrm{KO} \mathrm{NE}$ in the upper chamber comprising a time window from day 7-12 of differentiation to induce neuronal network formation of GNPs (Figure S8B). Co-culturing of GNPs with differentiating WT NE resulted in extensive neuronal network formation, while neuronal network formation was severely impaired when GNPs were co-cultured with Dnttipl KO and Elmsan1 KO NE (Figure S8C, D). In summary, these results suggest that the loss of MiDAC function in NE results in a lack of certain secretome components that are otherwise required for proper neurite outgrowth. To further establish the specific effects of MiDAC on the SLIT3 and NTN1 signaling pathways, we investigated the ligand-receptor pairs SLIT3/ROBO3 and NTN1/UNC5B. Importantly, compared to lysates from WT NE, lysates from Dnttipl KO NE showed strong reductions in SLIT3 and NTN1, whereas ROBO3 and UNC5B were not significantly affected (Figure 6A). Furthermore, we also observed a significant reduction of SLIT3 and NTN1 in CM of Dnttip1 KO versus WT NE (Figure 6A). The downstream effectors DAB1 of the NTN pathway and FAK of the SLIT/NTN signaling cascade were also deactivated, as shown by decreased phosphorylation of DAB1 and FAK in Dnttipl KO versus WT NE, despite no change in total protein levels (Figure 6A) (Zelina, Blockus et al., 2014). Having established that the SLIT3 and NTN1 signaling cascades are directly targeted by MiDAC during neurogenesis, we sought to test whether the loss of activity of these pathways is sufficient to explain the neurite outgrowth defects detected in neurons that had lost MiDAC function. To address this question, we differentiated Dnttipl KO mESCs into NE for 12 days and supplemented the CM from day 7 onward with recombinant SLIT3, NTN1, or a combination of SLIT3 and NTN1 (Figure S8A). To assess the specific effects of these ligands with their corresponding pathways, we combined the supplementation of CM with recombinant ligands with a blocking approach involving antibodies directed against the extracellular domains of either ROBO3 (SLIT3 pathway), UNC5B (NTN1 pathway), or a combination of ROBO3/UNC5B (Figure S8A). We found that SLIT3, NTN1, and SLIT3/NTN1 were all able to largely restore the neurite outgrowth defects observed in Dnttipl KO neurons and that the restoration of the phenotype was directly mediated by signaling through the ROBO3 and UNC5B signaling receptors (Figure 6B-D). We also obtained similar results using a modified version of the chamber assay described above, in which GNPs were treated with CM of WT or Dnttipl KO NE and identical combinations of recombinant ligands and blocking antibodies (Figure S8B). The reduced neuronal network formation, that was observed when GNPs were 
treated with CM of Dnttip1 KO NE, was largely rescued in the presence of SLIT3, NTN1, or SLIT3/NTN1, and the restoration of network formation was significantly abrogated by preblocking GNP-derived neurons with neutralizing antibodies against ROBO3 and/or UNC5B before ligand-containing CM of Dnttipl KO NE was added (Fig. S8E, F). Taken together, these findings indicate that the loss of MiDAC function in NE during neural differentiation results in strongly reduced SLIT3 and NTN1 signaling which accounts for the majority of the observed neurite outgrowth defects. 


\section{Discussion}

Here, we report that in mESCs, DNTTIP1 in association with ELMSAN1 and HDAC1, forms a chromatin-associated mitotic deacetylase complex called MiDAC. We provide evidence that MiDAC is enriched at promoters and enhancers genome-wide and can function as an activator and repressor of transcription in mESCs. More specifically, we uncover a crucial role for MiDAC in regulating a neurodevelopmental gene expression program that controls neurite outgrowth and network formation during neurogenesis. MiDAC achieves this by positively regulating a set of neurodevelopmental genes including genes of the axon guidance ligands SLIT3 and NTN1, while at the same time suppressing negative regulators of neurogenesis such as Spry4 and IdI. The results presented here support a model in which MiDAC is required to activate the promoters and enhancers of pro-neural genes such as Slit3 and Ntn1 while in parallel repressing negative regulators of neurogenesis by removing $\mathrm{H} 3 \mathrm{~K} 27 \mathrm{ac}$ from promoters and enhancers of genes such as Spry 4 and $I d 1$ to allow neurons to attain their required neurite length during differentiation (Figure 7).

MiDAC originally obtained its name from a chemoproteomic profiling study due to its increased association with certain HDAC inhibitors in protein extracts from mitotically arrested versus nonsynchronized proliferating cells that otherwise displayed comparable levels of DNTTIP1 (Bantscheff et al., 2011). Individual MiDAC subunits have been shown to interact with CCNA2 and/or the cell cycle-dependent kinase CDK2, and were reported to positively regulate the cell cycle inhibitors CDKN1A and CDKN1B and cell growth in various cancer cell lines, thus implying a potential role for MiDAC in cell cycle regulation (Gizard et al., 2005, Gizard et al., 2006, Hein et al., 2015, Huttlin et al., 2015, Pagliuca et al., 2011, Sawai et al., 2018, Zhang et al., 2018). However, we did not observe any effect on cell proliferation, cell cycle distribution, or any transcriptional changes in the above-mentioned cell cycle-related genes (Ccna2, Cdkn1a, Cdkn1b) in mESCs with a loss in MiDAC function, arguing in favor of a cell- and/or context-specific role for MiDAC in controlling cell proliferation and the cell cycle.

The C-terminus of DNTTIP1, subunit of MiDAC, has been reported to bind to a specific DNA binding motif following ChIP-seq studies utilizing a FLAG-tagged version of DNTTIP1 in HEK293 cells (motif: TGCAGTG-(14 bp)-CACTGCA flanked by AT-tracts) (Koiwai et al., 2015). In concordance with these results, our study confirms a chromatin-associated function and 
binding of DNTTIP1 to both promoter-proximal and -distal elements in mESCs. Although we did not find evidence of a clear DNTTIP1 consensus binding motif, we did detect a significant enrichment of several TF motifs at DNTTIP1-bound sites (data not shown). This implies that MiDAC is specifically recruited to chromatin by TFs and that its ability to bind nucleosomes might aid in MiDAC spreading from its initial recruitment site to carry out histone deacetylation. Furthermore, motif analysis of differentially expressed genes that display DNTTIP1 enrichment at their promoters or enhancers revealed the binding motifs of several TFs with previously reported roles in neurogenesis. We postulate that MiDAC upregulates a transcriptional program that is driven by ELK1, a member of the ETS family of pioneer transcription factors, while downregulating genes through RBFOX2, which negatively affects neurodevelopmental processes (Besnard et al., 2011, Gehman et al., 2012). However, it remains to be determined whether these TFs affect MiDAC recruitment and/or function during neurogenesis. Although DNTTIP1 levels are strongly reduced in Elmsan $1 \mathrm{KO}$ mESCs, we still observed some DNTTIP1 recruitment to chromatin. With TRERF1 and ZFP541 (ZNF541 in humans) two ELMSAN1-related proteins have been described that could also potentially be incorporated into MiDAC (Choi et al., 2008, Gizard et al., 2001). While ZFP541 is only very tissue-specifically expressed during spermatogenesis and not in mESCs, TRERF1 expression can be detected in mESCs (data not shown) (Choi et al., 2008). Thus, the remaining DNTTIP1 in Elmsan1 KO mESCs might form an alternative MiDAC complex with the ELMSAN1 paralog TRERF1 which could account for the residually detected DNTTIP1 recruitment in the absence of ELMSAN1.

HDAC1/2-containing complexes are generally considered repressors of transcription (Kadosh \& Struhl, 1998, Rundlett et al., 1998, Yang et al., 1996). However, accumulating evidence suggests that HDAC1/2 might also be involved in transcriptional activation (Bernstein et al., 2000, Harrison et al., 2011, Yamaguchi et al., 2010). Moreover, genome-wide studies have shown that HDAC1/2 localize to promoters, gene bodies, and enhancers of actively transcribed genes, thus implicating HDAC1/2 as positive regulators of transcription (Wang et al., 2009). Here, our finding that a high percentage of DNTTIP1-bound loci is associated with both down- and upregulated DEGs in Dnttipl KO versus WT mESCs and NE shows for the first time a direct role for MiDAC in both transcriptional activation and repression. The exact mode of action whereby MiDAC achieves activation or repression has yet to be elucidated in greater detail, but our studies provide evidence for at least two possible scenarios. First, MiDAC could control gene activation and repression by 
deacetylating histones. Most histone acetylation marks, including H3K27ac, correlate with transcriptional activation, but recently H4K20ac has been reported as a mark that is associated with silenced genes (Kaimori et al., 2016). Our data indicate that MiDAC might function as a repressor of genes that are marked by $\mathrm{H} 3 \mathrm{~K} 27 \mathrm{ac}$ and more broadly as an activator on repressed genes by mediating deacetylation of H4K20. As a second possibility, MiDAC might exert its function by deacetylating TFs or other chromatin-modifying proteins such as histone acetyltransferases (HATs). If specific TFs or HATs are negatively regulated by deacetylation through MiDAC at distinct target sites, this could also potentially explain the increase in H3K27ac (through $\mathrm{CBP} / \mathrm{p} 300$ ) or other histone acetylation marks on enhancers and promoters of up- or downregulated genes between WT, Dnttipl KO and Elmsan1 KO mESCs and NE.

HDAC1 and HDAC2 are important regulators of many developmental processes including neurogenesis (Kelly \& Cowley, 2013). But their function remains enigmatic as they can be recruited into at least four independent HDAC1/2-containing complexes (Millard et al., 2017). While the HDAC1/2-containing SIN3, NuRD and CoREST complexes have been implicated in neurogenesis, the physiological function of MiDAC has been unexplored to date (Andres et al., 1999, Knock et al., 2015, Nitarska et al., 2016, Wang et al., 2016). Here, we report that MiDAC directly controls a neurodevelopmental gene expression program and specifically targets enhancers and promoters of positive and negative regulators that are involved in neurite outgrowth during neural differentiation. While this study emphasizes the role of MiDAC in neural differentiation and neurite outgrowth, it is evident from our gene expression analyses that this neurodevelopmental gene expression program only constitutes a fraction of the MiDAC-regulated transcriptome in mESCs pointing to other biological roles of MiDAC. SLIT/ROBO, EPHRIN/EPH, NETRIN/DCC/UNC, and SEMAPHORIN/PLEXIN signaling represent the four major canonical axon guidance pathways (Bashaw \& Klein, 2010). Integration of our gene expression and ChIP data showed that MiDAC directly activates the genes of the axon guidance ligands SLIT3 and NTN1 while repressing the genes of the negative regulators SPRY4 and ID1. SLIT3 and NTN1 are ligands for their cognate receptors ROBO3 and UNC5B, respectively. Recombinant SLIT3 and NTN1 either alone or in combination were able to largely rescue the neurite outgrowth defects in neurons that have lost MiDAC subunits. Interestingly, blocking only ROBO3 or only UNC5B signaling was sufficient to inhibit the rescue effects of SLIT3 in combination with NTN1, suggesting that a heteromeric ROBO3/UNC5B axis is required for 
efficient transduction of SLIT3/NTN1 signaling to ensure proper neurite outgrowth. While evidence exists that heteromeric receptor association and signaling of ROBO3/UNC5B occurs, it has yet to be determined whether this also applies to the role of MiDAC in an in vivo system (Zelina et al., 2014). In summary, while individual TFs have been reported to transcriptionally regulate select axon guidance ligands or receptors little is known about their regulation through chromatin-modifying components (Kim, Kim et al., 2016, Labrador, O'Keefe et al., 2005). Here, we describe how the axon guidance ligands SLIT3 and NTN1 are transcriptionally controlled by an epigenetic regulator, the MiDAC complex. Finally, neurite outgrowth defects are observed in several neurodevelopmental disorders such as autism, epilepsy, fragile X-syndrome and psychiatric disorders (Gilbert \& Man, 2017, Huang \& Song, 2019, Krejci, Legartova et al., 2017, Wen, Cheng et al., 2017). However, the underlying molecular mechanisms remain unclear. Our study implicates MiDAC as an essential regulator of neurite outgrowth which suggests that it might be playing an important role in certain neurodevelopmental disorders. Future studies of MiDAC in the context of neurodevelopmental disorders are thus likely to yield insightful mechanistic details about disease pathogenesis. 


\section{Materials and Methods}

\section{Cell Lines}

Male C57/BL6 Bruce-4 mESCs (Millipore, SF-CMTI-2) and their Dnttip1 KO and Elmsan1 KO derivatives were originally cultured under feeder free culture conditions (KnockOut DMEM medium) on $0.1 \%$ gelatin-coated flasks or plates. After CRISPR/Cas9 genome-editing mESCs were transitioned to chemically defined naïve culture conditions (2iL) on $0.1 \%$ gelatin-coated flasks or plates.

Feeder-free culture conditions (SL):

KnockOut DMEM (no L-glutamine); 15\% FBS (ES cell qualified); $2 \mathrm{mM} \mathrm{L-Glutamine} \mathrm{or}$ GlutMAX (1:100); $1 \mathrm{mM}$ Sodium Pyruvate (1:100); $0.1 \mathrm{mM}$ MEM Non-Essential Amino Acids (1:100); Embryomax Nucleosides (1:100); $0.1 \mathrm{mM} \beta$-Mercaptoethanol (1:500); $1000 \mathrm{U} / \mathrm{ml} \mathrm{LIF}$ (1:10,000 from $10^{7} \mathrm{U} / \mathrm{ml}$ stock); $50 \mathrm{U} / \mathrm{ml} \mathrm{Pen/Strep} \mathrm{(1:100).}$

Chemically defined naïve culture conditions (2iL):

50\% DMEM/F-12; 50\% Neurobasal Medium (no L-glutamine); B-27 Supplement, minus vitamin A (1:100); N-2 Supplement (1:200); 2 mM L-Glutamine or GlutaMAX (1:100); $0.1 \mathrm{mM} \beta$ Mercaptoethanol (1:500); $3 \mu \mathrm{M}$ CHIR 99021 (GSK3 $\beta$ inhibitor) (1:1000 from $3 \mathrm{mM}$ stock in DMSO); $1 \mu \mathrm{M}$ PD 0325901 (MEK inhibitor) (1:1000 from $1 \mathrm{mM}$ stock in DMSO); $1000 \mathrm{U} / \mathrm{ml}$ LIF (1:10,000 from $10^{7} \mathrm{U} / \mathrm{ml}$ stock); $50 \mathrm{U} / \mathrm{ml}$ Pen/Strep (1:100).

\section{Neural Differentiation}

\section{Directed differentiation into neuro-ectoderm}

WT, Dnttipl KO and Elmsan1 KO mESCs were dissociated by trypsinization, resuspended in differentiation medium 1 and pelleted by centrifugation. 1 x $10^{6} \mathrm{mESCs}$ were resuspended in $3 \mathrm{ml}$ differentiation medium 1 and then transferred into one well of 6-well ultra-low attachment plate (Corning, 3471). Cells were grown in differentiation medium 1 for 3 days. The differentiation medium 1 was changed every day. After 3 days the emerging embryoid bodies (EBs) were cultured in differentiation medium 2 containing $2 \mu \mathrm{M}$ retinoic acid (Santa Cruz Biotechnology, sc-200898) for an additional 3 days. The differentiation medium 2 was changed every day. After 6 days the EBs were picked up gently with a $200 \mu 1$ large orifice tip and transferred into a $1.5 \mathrm{ml}$ tube. The 
EBs were allowed to settle down by gravity followed by careful aspiration of the supernatant. 1 $\mathrm{ml}$ differentiation medium 3 containing $20 \mathrm{ng} / \mathrm{ml} \mathrm{FGF2}$ and $20 \mathrm{ng} / \mathrm{ml}$ EGF was carefully added to the $1.5 \mathrm{ml}$ tube containing the EBs, and the EBs were gently picked up with a $200 \mu 1$ large orifice tip and transferred onto a $0.1 \%$ gelatin-coated well of a standard 6-well plate (Corning, 3516). Subsequently, $1 \mathrm{ml}$ of additional differentiation medium 3 was carefully added to the well containing the EBs. The EBs were allowed to differentiate for the next 6 days, with daily changing of differentiation medium 3. The EBs will attach slowly over time and aster-shaped neurons will emerge from the periphery of the EBs forming increasingly longer neurite extensions and networks. Neurite outgrowth was quantified and visualized after 12 days of differentiation. Experiments to rescue the neurite outgrowth defects of Dnttipl KO and Elmsan $1 \mathrm{KO}$ neurons with CM from WT NE were conducted as above with the following alterations. After 6 days of differentiation all Dnttipl KO and Elmsan1 KO CM was removed and replaced daily with 50\% fresh differentiation medium 3 and 50\% CM from WT NE for the next 6 days. Experiments to rescue the neurite outgrowth defects of Dnttipl KO neurons with axon guidance ligands were carried out as described above except that recombinant SLIT3 (R\&D Systems, 9296-SL-050), NTN1 (R\&D Systems, 6419-N1-025) or a combination of SLIT3/NTN1 were added daily to differentiation medium 3 (at a concentration of 0.5 and $0.25 \mathrm{ug} / \mathrm{ml}$ for SLIT3 and NTN1 respectively) from day 6 of differentiation onward. The rescued neurite outgrowth defects of Dnttip1 KO neurons via SLIT3, NTN1 or SLIT3/NTN1 supplementation were blocked by using antibodies directed against the extracellular domain of the axon guidance receptors ROBO3 (R\&D Systems, AF3155), UNC5B (R\&D Systems, MAB1006), a combination of ROBO3/UNC5B or IgG (Millipore, 12-370) as a negative control. Blocking was performed daily from day 7 onward by adding $0.5 \mu \mathrm{g}$ of each antibody to differentiation medium 3 two hours before addition of the ligands SLIT3, NTN1 or SLIT3/NTN1.

Differentiation medium 1:

50\% DMEM/F-12; 50\% Neurobasal Medium (no L-glutamine); B-27 Supplement Supplement with vitamin A, serum free (1:100); N-2 Supplement (1:200); 0.1 mM $\beta$-Mercaptoethanol (1:500); $50 \mathrm{U} / \mathrm{ml}$ Pen/Strep (1:100).

Differentiation medium 2: 
Differentiation medium $1 ; 2 \mu \mathrm{M}$ retinoic acid.

Differentiation medium 3:

Differentiation medium 1; $20 \mathrm{ng} / \mathrm{ml} \mathrm{FGF2;} 20 \mathrm{ng} / \mathrm{ml} \mathrm{EGF.}$

Chamber assay to assess neuronal network formation from granule neuron progenitor cells (GNPs)

GNPs were isolated from mouse cerebella from postnatal day 6-7 (a gift from Martine Roussel) (Vo, Wolf et al., 2016). GNPs were grown as neurospheres in GNP medium in 6-well ultra-low attachment plates (Corning, 3471). GNP neurospheres were dissociated by trypsinization, resuspended in GNP culture medium and pelleted by centrifugation. $10^{5}$ GNPs were resuspended in $600 \mu \mathrm{l} \mathrm{GNP}$ culture medium and seeded into one well of a 24-well ultra-low attachment plate (Corning, 3473) and allowed to form neurospheres for 3 days. GNP culture medium was changed every day. From this point onward GNPs were differentiated in the same fashion as described in "directed differentiation into neuro-ectoderm" by sequential culturing in differentiation medium 2 and 3 and adjustment of media volumes to a 24-well format (one fifth of a 6-well format) with the following alterations. After culturing in differentiation medium 2 GNPs were plated into one well of a 24-well transwell chamber (pre-coated with 1\% gelatin) in differentiation medium 3 (Corning, 354480) and differentiated for another 6 days. WT, Dnttipl KO and Elmsan1 KO mESCs were differentiated according to the "Directed differentiation into neuro-ectoderm" protocol for the first 6 days but experiments were scaled down so that EBs were grown in one well of a 24-well ultralow attachment plate (Corning, 3473) and all media volumes were adjusted to one fifth of the 6well protocol. The EBs were carefully transferred into a matrigel-coated insert and allowed to differentiate for the next 6 days, with daily changing of differentiation medium 3 in the insert and GNP medium in the bottom chamber. In parallel, GNP medium and MEF CM medium was added to separate inserts as positive and negative controls, respectively and media changes were carried out in the same way as for differentiation medium 3. Experiments to rescue the network formation defects of GNP-derived neurons caused by culturing in CM of Dnttipl KO NE were carried out as described above for the first six days of differentiation. However, instead of co-culturing GNPs with Dnttip1 KO NE, GNPs were supplemented daily with CM of Dnttip1 KO NE (50\%), 50\% fresh differentiation medium 3 and recombinant SLIT3 (R\&D Systems, 9296-SL-050), NTN1 
(R\&D Systems, 6419-N1-025) or a combination of SLIT3/NTN1 for 6 days from the day of GNP seeding. Network formation of GNP-derived neurons grown in CM of Dnttipl KO NE and differentiation medium 3 supplemented with SLIT3, NTN1 or SLIT3/NTN1 was blocked by using antibodies directed against the extracellular domain of the axon guidance receptors ROBO3 (R\&D Systems, AF3155), UNC5B (R\&D Systems, MAB1006), a combination of ROBO3/UNC5B or IgG (Millipore, 12-370) as a negative control. Blocking was performed daily for 6 days from the day of GNP seeding by aspirating the old medium and adding $0.1 \mu \mathrm{g}$ of each blocking antibody in fresh differentiation medium 3 two hours before adding CM of Dnttipl KO NE (50\%), 50\% fresh differentiation medium 3 with either the ligands SLIT3, NTN1 or SLIT3/NTN1. Network formation of GNP-derived mature neurons was quantified and visualized 6 days after GNPs were seeded. A neuronal network was scored when all neurite projections of an individual neuron were well connected with the neurites of neighboring neurons and formed a closed local circuit as assessed by TUBB3 staining (Doetsch \& Alvarez-Buylla, 1996, Shepherd, 1998). The percentage of network formation was scored as follows: =total number of complete networks per TUBB3positive neuron/total number of DAPI-positive cells) x 100. The different groups were statistically analyzed using ONE-way ANOVA t-test.

GNP medium:

Neurobasal medium (no L-glutamine); 2 mM L-Glutamine; B-27 Supplement with VitA, serum free (1:100); N-2 Supplement (1:200); 20 ng/ml FGF2; 20 ng/ml EGF; 50 U/ml Pen/Strep (1:100).

\section{Alkaline Phosphatase Staining and Quantification}

Alkaline phosphatase staining of mESCs was carried out with the Alkaline Phosphatase Detection Kit (Millipore, SCR004) following the manufacturer's instructions. $100 \mathrm{mESC}$ colonies were assessed per genotype.

\section{ChIP-seq Library Preparation and Sequencing}

DNA was quantified using the Quant-iT PicoGreen dsDNA Assay (Thermo Fisher Scientific, P11496). Libraries were prepared with the KAPA HyperPrep Library Kit (Roche, 07962363001) and analyzed for insert size distribution with the High Sensitivity DNA Kit (Agilent, 5067-4626) on a 2100 Bioanalyzer or the High Sensitivity D1000 ScreenTape Assay (Agilent, 5067-5584, 
5067-5585, 5067-5587, 5067-5603) on a 4200 TapeStation. Libraries were quantified using the Quant-iT PicoGreen dsDNA Assay. Single end 50 cycle sequencing was performed on a HiSeq 2500, HiSeq 4000, or NovaSeq 6000 System (all from Illumina).

\section{Chromatin Immunoprecipitation (ChIP)}

ChIPs were performed according to a modified version of Lee et al., 2006(Lee, Johnstone et al., 2006). For ChIP-seq applications of non-histone proteins ChIP was carried out with $5 \times 10^{7}$ cells and for histone ChIPs with 2-2.5 x $10^{7} \mathrm{mESCs}$. For manual ChIP applications of non-histone proteins ChIP was carried out with $2 \times 10^{6} \mathrm{NE}$ cells and for histone ChIPs with $10^{6} \mathrm{NE}$ cells. For non-histone ChIPs dual crosslinking was performed at RT for $30 \mathrm{~min}$ with $2 \mathrm{mM}$ disuccinimidyl glutarate (DSG) in DPBS followed by addition of paraformaldehyde to a final concentration of $1 \%$ and further crosslinking for 15 minutes. For histone ChIPs crosslinking was performed at RT for 15 minutes with 1\% paraformaldehyde. Crosslinking was quenched with $150 \mathrm{mM}$ glycine for 5 minutes at RT. After quenching the cells were pelleted, the supernatant aspirated and the cell pellet washed once in DPBS and then snap-frozen and stored at $-80^{\circ} \mathrm{C}$ or immediately further processed. The crosslinked cell pellet was carefully resuspended in Lysis Buffer 1 with a transfer pipet and the cell suspension incubated for 10 minutes on a nutator at $4^{\circ} \mathrm{C}$. Nuclei were then pelleted and the supernatant aspirated. The cell pelleted nuclei were carefully resuspended in Lysis Buffer 2 with a transfer pipet and the cell suspension incubated for 10 minutes on a nutator at $4^{\circ} \mathrm{C}$. Nuclei were then pelleted again and the supernatant aspirated. The nuclear pellet was then resuspended in Lysis Buffer 3 and the suspension sonicated with a probe sonicator (Fisher Scientific Model 705 Sonic Dismembrator) at output setting $55(27-33 \mathrm{~W})$ for 12 cycles with each cycle constituting a 30 second sonication burst followed by a 60 second pause. After sonication 1/20 volume of 20\% Triton X-100 was added and the sample was mixed. The chromatin was cleared by centrifugation for 10 minutes at $20,000 \mathrm{~g}$ at $4^{\circ} \mathrm{C}$ and the supernatant was transferred to a fresh tube. The cleared chromatin was then stored at $4^{\circ} \mathrm{C}$. For each ChIP $100 \mu$ l Protein A/G-Plus agarose (Santa Cruz Biotechnology, sc-2003) slurry was used. The beads were washed in $1 \mathrm{ml}$ Blocking Solution, pelleted and the supernatant was removed. The bead wash step was repeated another time. The beads were resuspended in $1 \mathrm{ml}$ Blocking Solution followed by addition of the appropriate antibody and were incubated on a nutator at $4{ }^{\circ} \mathrm{C}$ overnight. For ChIP-seq applications of nonhistone proteins and histones $10 \mu \mathrm{g}$ of antibody was used. For manual ChIP applications of non- 
histone proteins and histones $3 \mu \mathrm{g}$ of antibody was used. On the next day the stored chromatin was cleared another time by centrifugation for 10 minutes at $20,000 \mathrm{~g}, 4^{\circ} \mathrm{C}$ and the supernatant was transferred to a fresh tube. A small chromatin aliquot of $50 \mu \mathrm{l}$ was reserved as input control and was kept on ice until further processing (see below). The beads were washed twice with $1 \mathrm{ml}$ Blocking Solution as on the previous day and resuspended in $100 \mu$ l Blocking Solution. The cleared chromatin was added and incubated with the beads on a nutator for 3 hours at $4{ }^{\circ} \mathrm{C}$. After the incubation the beads were pelleted and the supernatant aspirated. $1 \mathrm{ml}$ Wash Buffer was added and the sample was mixed gently by inverting the tube several (8-10) times to ensure that all beads were fully resuspended. The beads were then pelleted and the supernatant aspirated. This wash step was repeated another 4 times ( 5 washes total). A final wash with $1 \mathrm{ml} \mathrm{TE} \mathrm{buffer} \mathrm{(10} \mathrm{mM} \mathrm{Tris}$ $\mathrm{HCl} \mathrm{pH}$ 8.0; 1 mM EDTA) was added (including mixing, pelleting of beads and aspiration of supernatant). Chromatin was eluted with $200 \mu$ Elution Buffer and the samples were incubated with shaking at $900 \mathrm{rpm}$ for 30 minutes at $65^{\circ} \mathrm{C}$ in a ThermoMixer (Eppendorf, 5382000023 with a ThermoTop (Eppendorf, 5308000003). The beads were then pelleted and the supernatant transferred to a fresh tube. The input sample was brought up to $200 \mu 1$ with Elution Buffer and all samples (input and ChIP samples) incubated overnight at $65^{\circ} \mathrm{C}$ (in a ThermoMixer with a ThermoTop) to reverse crosslinks. On the next day $200 \mu \mathrm{l}$ TE and $8 \mu 1$ of $10 \mathrm{mg} / \mathrm{ml}$ RNaseA were added followed by incubation in a ThermoMixer with a ThermoTop for 1 hour at $37^{\circ} \mathrm{C}$. Next, 10 $\mu 120 \mathrm{mg} / \mathrm{ml}$ Proteinase $\mathrm{K}$ was added and the sample was incubated for an additional 2 hours at $55^{\circ} \mathrm{C}$ in a ThermoMixer with a ThermoTop. DNA was purified following the instructions of the QIAquick PCR Purification Kit from Qiagen (Qiagen, 28106). Elution of DNA was performed with $50 \mu 1$ of prewarmed $\left(55^{\circ} \mathrm{C}\right) \mathrm{EB}$ buffer.

Lysis Buffer 1:

50 mM HEPES KOH pH 7.5; 140 mM NaCl ; 1 mM EDTA; 10\% Glycerol; 0.5\% NP-40 (Igepal CA-630); 0.25\% Triton X-100; protease inhibitors (Sigma, P8340) (1:200).

Lysis Buffer 2:

10 mM Tris $\mathrm{HCl}$ pH 8.0; 200 mM NaCl; 1 mM EDTA; 0.5 mM EGTA; protease inhibitors (Sigma, P8340) (1:200). 
Lysis Buffer 3:

10 mM Tris $\mathrm{HCl}$ pH 8.0; 100 mM NaCl; 1 mM EDTA; 0.5 mM EGTA; 0.1\% Na-deoxycholate; $0.5 \%$ N-lauroylsarcosine; protease inhibitors (Sigma, P8340) (1:200).

Blocking Solution:

DPBS (Thermo Fisher Scientific, 14190144); 0.5\% BSA (Sigma, A7906); 0.1\% Triton X-100; protease inhibitors (Sigma, P8340) (1:500).

Wash Buffer:

50 mM HEPES KOH pH 7.5; 500 mM LiCl; 1 mM EDTA; 1\% NP-40 (Igepal CA-630); 0.7\% Nadeoxycholate; protease inhibitors (Sigma, P8340) (1:500).

Elution Buffer:

$50 \mathrm{mM}$ Tris $\mathrm{HCl} \mathrm{pH}$ 8.0; 10 mM EDTA; 1\% SDS.

\section{Flow Cytometry}

mESC colonies were dissociated by trypsinization, resuspended in $2 \mathrm{iL}$ medium and pelleted by centrifugation. Cells were washed once in DPBS followed by aspiration of the supernatant. While the cells were gently vortexed to avoid clumping, fixation was performed by dropwise addition of cold $70 \%$ ethanol. After fixation for 30 minutes on ice, the cells were pelleted by centrifugation and the supernatant aspirated. The cell pellet was then washed in DPBS and pelleted by centrifugation followed by aspiration of the supernatant. The cell pellet was resuspended in $50 \mu 1$ of a $100 \mu \mathrm{g} / \mathrm{ml}$ RNase solution to ensure that only DNA, not RNA, is stained. $200 \mu 1$ propidium iodide (PI) from a $50 \mu \mathrm{g} / \mathrm{ml}$ stock solution was added to each sample to stain the DNA. Cell cycle analysis of mESCs was carried out on a BD LRPFortessa cell analyzer. For cytometry analysis of PAX6-positive neural progenitor cells and TUBB3-positive neurons from 8 and 12 day old NE respectively, PAX6/DAPI and TUBB3/DAPI IF staining of NE was carried out as described under "Immunofluorescence (IF)" followed by trypsinization and FACS analysis. PAX6- and TUBB3positive cells were sorted with a BD FACSAria III cell sorter and the DNA content of individual cells was determined as a readout of DAPI intensity. Bar plots were generated from the raw data. Experiments were conducted in triplicate for cell cycle analysis of mESCs. 


\section{Immunofluorescence (IF)}

Neural differentiation was carried out in wells of a 6-well or 24-well plate as described in "Experimental Models" under "Neural Differentiation". Fixation Solution containing 8\% paraformaldehyde was prepared from 16\% paraformaldehyde (Electron Microscopy Sciences, 15710) and $10 \mathrm{x}$ DPBS (Thermo Fisher Scientific, 14200075) and adjusted with $\mathrm{H}_{2} \mathrm{O}$ to achieve a final concentration of DPBS. $1 \mathrm{ml} / 200 \mu \mathrm{l}$ (6-well/24-well) Fixation Solution was added to 1 $\mathrm{ml} / 200 \mu \mathrm{l}$ (6-well/24-well) of remaining culture medium and cells were incubated for 20 minutes at RT. The fixative was aspirated and rinsed with $2 \mathrm{ml} / 400 \mu \mathrm{l}$ (6-well/24-well) DPBS (Thermo Fisher Scientific, 14190144) and the DPBS aspirated again. Permeabilization was performed with $1 \mathrm{ml} / 200 \mu \mathrm{l}$ (6-well/24-well) Permeabilization Solution for 10 minutes at room temperature. Following aspiration of the Permeabilization Solution the cells were rinsed three times with $1 \mathrm{ml}$ Wash Buffer and aspiration of the Wash Buffer after each rinse. Blocking was performed with 1 $\mathrm{ml} / 200 \mu \mathrm{l}$ (6-well/24-well) Blocking Buffer for one hour at room temperature. Blocking Buffer was aspirated and $500 \mu \mathrm{l} / 100 \mu \mathrm{l}$ (6-well/24-well) Wash Buffer containing the primary antibody was added and the sample was incubated in a humidified light-tight chamber at $4^{\circ} \mathrm{C}$ overnight. On the next day Wash Buffer containing the primary antibody was aspirated. The sample was then washed 3 times for 5 minutes with $2 \mathrm{ml} / 400 \mu \mathrm{l}$ (6-well/24-well) Wash Buffer with gentle shaking on a rotator at room temperature and aspiration of the Wash Buffer after each step. $500 \mu 1 / 100 \mu 1$ (6-well/24-well) Wash Buffer containing the fluorochrome-conjugated secondary antibody and DAPI ( $1 \mu \mathrm{g} / \mathrm{ml}$ final concentration) was added and the sample was incubated with gentle shaking on a rotator in a humidified light-tight chamber for one hour at room temperature. The sample was again washed 3 times for 5 minutes with 2 ml/400 $\mu$ l (6-well/24-well) Wash Buffer with gentle shaking on a rotator at room temperature and aspiration of the Wash Buffer after each step. 1 $\mathrm{ml} / 200 \mu \mathrm{l}$ (6-well/24-well) DPBS was added and the sample was imaged with an inverted widefield microscope platform (Leica, DMi8).

Antibody dilutions for IF:

Mouse $\alpha$-MAP2 (Sigma, M9942); 1:1000. Mouse $\alpha$-TUBB3 (BioLegend, 801201); 1:1000.

Fixation Solution: 
DPBS; 8\% paraformaldehyde

Permeabilization Solution:

DPBS; $0.2 \%$ Triton X-100

Blocking Buffer:

DPBS; $50 \mathrm{mg} / \mathrm{ml} \mathrm{BSA} \mathrm{(5 \% );} \mathrm{0.1 \%} \mathrm{goat} \mathrm{serum.}$

Wash Buffer:

DPBS; $5 \mathrm{mg} / \mathrm{ml} \mathrm{BSA}(0.5 \%)$

\section{Immunoprecipation (IP)}

$2 \times 10^{7} \mathrm{mESCs}$ were used for each IP. mESCs were scraped off with a cell scraper and pelleted by centrifugation. The cell pellet was resuspended in DPBS and pelleted again. Cytoplasmic lysis was performed in $1 \mathrm{ml}$ Lysis Buffer 1 by resuspending the cell pellet followed by incubation on a nutator for 5 minutes at $4{ }^{\circ} \mathrm{C}$. Nuclei were centrifuged for 5 minutes at $1500 \mathrm{~g}$ at $4^{\circ} \mathrm{C}$, the supernatant (cytoplasmic fraction) aspirated and the nuclear pellet resuspended in $500 \mu 1$ Lysis Buffer 2. The protein concentrations of the nuclear extracts were determined by a protein assay (BioRad, 5000006) with a spectrophotometer (Eppendorf, 6133000010) using disposable cuvettes (Eppendorf, 0030079353). Equal protein amounts were used for all samples of the same IP experiment and volumes were adjusted to $545 \mu 1$ with Lysis Buffer 2. 45 ul of each sample was removed and used as Input. For each IP $50 \mu 1$ Dynabeads Protein G (Thermo Fisher Scientific, 10004D) slurry was used. The beads were washed in $1 \mathrm{ml}$ Lysis Buffer 2, collected with a magnetic stand (Thermo Fisher Scientific, 12321D) and the supernatant was removed. The bead wash step was repeated another time. The beads were resuspended in $500 \mu$ Lysis Buffer 2 followed by addition of $5 \mu \mathrm{g}$ of the appropriate antibody and were incubated for two hours on a nutator at $4^{\circ} \mathrm{C}$. The beads were collected with a magnetic stand and the supernatant was aspirated followed by two wash steps in $500 \mu \mathrm{l}$ Lysis Buffer 2. The nuclear extracts were added to the beads and incubated with the beads on a nutator at $4^{\circ} \mathrm{C}$ overnight. After the incubation the beads were collected with a magnetic stand and the supernatant aspirated. $1 \mathrm{ml}$ Lysis Buffer 2 was added and the sample was mixed gently by inverting the tube until all beads were fully resuspended. The 
beads were then collected with a magnetic stand and the supernatant aspirated. This wash step was repeated another 2 times ( 3 washes total). The beads were resuspended in $50 \mu 1$ of 1 x SDS Laemmli Buffer and the inputs with 15 ul 4 x SDS Laemmli Buffer and all samples were boiled for 5 minutes at $95^{\circ} \mathrm{C}$ on a heating block (Thermo Fisher Scientific, 88870003). Inputs were centrifuged in a table top centrifuge for 5 minutes at full speed while bead IP samples were centrifuged for 2 minutes at $850 \mathrm{~g}$, RT. Equal volumes of Input and IP samples were loaded and separated on a 4-20\% gradient SDS-PAGE gel (Bio-Rad, 4561096 and 4561093) in running buffer (Bio-Rad, 1610772) using a Bio-Rad electrophoresis and blotting system (Bio-Rad, 1658033). Western blotting was carried out as described under "Western Blotting".

Lysis Buffer 1:

10 mM HEPES pH 7.9; 10 mM KCl; 1.5 mM MgCl $2 ; 0.5 \%$ Igepal CA-630 (NP-40); 0.5 mM DTT; protease inhibitors (Sigma, P8340) (1:200).

Lysis Buffer 2:

20 mM HEPES pH 7.9; 420 mM NaCl; 1.5 mM MgCl $2 ; 0.2$ mM EDTA; $10 \%$ glycerol; $0.5 \mathrm{mM}$ DTT; protease inhibitors (Sigma, P8340) (1:200).

4 x SDS Laemmli Buffer:

$250 \mathrm{mM}$ Tris $\mathrm{pH}$ 6.8, 50\% Glycerol, 8\% SDS, 0.008\% Bromophenol blue

1 x SDS Laemmli Buffer:

$900 \mu 14$ X SDS Laemmli Buffer Stock + $100 \mu 1 \beta$-Mercaptoethanol

\section{Proliferation Assay}

$5 \times 10^{4}$ mESCs (WT, Dnttipl KO and Elmsanl KO) per well were plated in a 24-well plate and proliferation was monitored over a period of 5 days. From day two onward mESCs were harvested and counted with an automated cell counter (Thermo Fisher Scientific, Countess II FL Automated Cell Counter). For each time point and clone experiments were repeated in triplicate. For statistical analysis unpaired Student's t-test was performed between WT and Dnttipl KO/Elmsan1 KO mESCs. 


\section{qPCR}

qPCR reactions were carried out with SYBR ${ }^{\mathrm{TM}}$ Green PCR Master Mix (Thermo Fisher Scientific, 4309155) on a QuantStudio 7 Flex Real-Time PCR System (Thermo Fisher Scientific) in 384-well format (Thermo Fisher Scientific, 4309849) in a total reaction volume of $10 \mu \mathrm{l}$ with $1 \mu \mathrm{l}$ of undiluted eluted ChIP DNA and a final primer concentration of $200 \mathrm{nM}$. qPCR primers used in this study are shown in Table S6. The calculation and analysis for \% Input was as follows(Dahl \& Collas, 2008): \% Input=[(Amount of ChIP DNA)/(Amount of Input DNA x Dilution Factor) $]$ x 100

\section{RNA Isolation}

RNA was isolated from $3 \times 10^{6}$ cells with the RNeasy Mini Kit (Qiagen, 74106) following the manufacturer's instructions with the following alterations. Cells were resuspended in $600 \mu \mathrm{RLT}$ buffer (with 2-Mercaptoethanol) and the homogenate further passed through a QiaShredder column (Qiagen, 79656) by centrifugation in a table top centrifuge for 2 minutes at full speed at room temperature (RT). The optional step after the second wash with RPE buffer was applied to dry the membrane. RNA was eluted with $85 \mu 1 \mathrm{H}_{2} \mathrm{O}$ and supplied with $10 \mu 1$ 10x DNAase buffer and $5 \mu \mathrm{l}$ DNAse I (NEB, M0303S), mixed and incubated at RT for $20 \mathrm{~min}$. After incubation RNA was purified by following the "RNA Cleanup" protocol in the RNeasy Mini Handbook. The optional step after the second wash with RPE buffer was applied to dry the membrane. RNA was eluted in $50 \mu \mathrm{l} \mathrm{H}_{2} \mathrm{O}$ and concentration determined with a NanoDrop 8000 Spectrophotometer.

\section{RNA-seq Library Preparation and Sequencing}

RNA was quantified using the Quant-iT RiboGreen RNA Assay Kit (Thermo Fisher Scientific, R11490) and quality checked with the RNA 6000 Nano Kit (Agilent, 5067-1511) on a 2100 Bioanalyzer (Agilent, G2939BA) or High Sensitivity RNA ScreenTape Assay (Agilent, 50675579 , 5067-5580, 5067-5581) on a 4200 TapeStation (Agilent, G2991AA) prior to library generation. Libraries were prepared from total RNA with the TruSeq Stranded Total RNA Library Prep Gold Kit (Illumina, 20020599) according to the manufacturer's instructions. Libraries were analyzed for insert size distribution with the High Sensitivity DNA Kit (Agilent, 5067-4626) on a 2100 Bioanalyzer or the High Sensitivity D1000 ScreenTape Assay (Agilent, 5067-5584, 5067- 
$5585,5067-5587,5067-5603)$ on a 4200 TapeStation. Libraries were quantified using the QuantiT PicoGreen dsDNA Assay (Thermo Fisher Scientific, P11496). Paired end 100 cycle sequencing was performed on a HiSeq 2500, HiSeq 4000, or NovaSeq 6000 System (all from Illumina) according to the manufacturer's instructions.

\section{qRT-PCR}

qRT-PCR reactions were carried out with the Power SYBR ${ }^{\mathrm{TM}}$ Green RNA-to- $\mathrm{C}_{\mathrm{T}}^{\mathrm{TM}}$ 1-Step Kit (Thermo Fisher Scientific, 4389986) on a QuantStudio 7 Flex Real-Time PCR System (Thermo Fisher Scientific) in 384-well format (Thermo Fisher Scientific, 4309849) in a total reaction volume of $10 \mu \mathrm{l}$ with $40 \mathrm{ng}$ of RNA and a final primer concentration of $200 \mathrm{nM}$. qRT-PCR primers used in this study are shown in Table S5. Analysis was performed applying the delta-delta $\mathrm{Ct}$ method (2- $\left.2^{-\Delta \Delta \mathrm{Ct}}\right)$ as previously described(Livak \& Schmittgen, 2001).

\section{Western Blotting}

Whole cell lysates from mESCs or differentiated neuroectoderm were obtained according to the following protocol. Medium was aspirated, DPBS was added and cells were detached by resuspension with a pipet or a cell scraper and transferred into a fresh tube. After centrifugation the supernatant was aspirated and the pellet resuspended in $1 \mathrm{ml}$ ice cold DPBS. After repelleting in a cooled table top centrifuge and aspiration of the supernatant the cell pellet was resuspended in RIPA buffer followed by incubation on a nutator for 30 minutes at $4^{\circ} \mathrm{C}$. The resulting cell lysate was centrifuged in a cooled table top centrifuge for 5 minutes at full speed and transferred to a new tube. The protein concentration of the supernatant was determined by a protein assay (BioRad, 5000006) with a spectrophotometer (Eppendorf, 6133000010) using disposable cuvettes (Eppendorf, 0030079353). The protein concentration was adjusted to a desired final protein concentration of 1-2 $\mu \mathrm{g} / \mu \mathrm{l}$ with 4 x SDS Laemmli Buffer and additional RIPA Buffer to achieve a final 1 x SDS Laemmli Buffer concentration. 1 x SDS Laemmli Buffer samples were boiled for 5 minutes at $95^{\circ} \mathrm{C}$ on a heating block (Thermo Fisher Scientific, 88870003) and centrifuged in a table top centrifuge for 5 minutes at full speed, RT. From the supernatant equal amounts of protein (usually 10-30 $\mu \mathrm{g}$ ) were loaded and separated on a 4-20\% gradient SDS-PAGE gel (Bio-Rad, 4561096 and 4561093) in running buffer (Bio-Rad, 1610772) using a Bio-Rad electrophoresis and blotting system (Bio-Rad, 1658033). Proteins were transferred to either a PVDF or nitrocellulose 
membrane (Santa Cruz Biotechnology, sc-3723 and sc-3724) with the same Bio-Rad system using Western Transfer Buffer (Bio-Rad, 1610771 and 20\% methanol) for 90 minutes at $400 \mathrm{~mA}$. The membranes were blocked for $1 \mathrm{~h}$ at room temperature with gentle rocking in 5\% dry milk in TBST buffer. Primary antibody incubation was performed overnight at $4{ }^{\circ} \mathrm{C}$ with gentle rocking in $5 \%$ dry milk in TBST Buffer. On the next day the membranes were washed three times for 5 minutes with $10 \mathrm{ml}$ TBST Buffer with gentle rocking and then incubated with horse radish peroxidase coupled secondary IgG-specific antibodies in TBST Buffer for $1 \mathrm{~h}$ at room temperature with gentle rocking. After an additional three washes for 5 minutes with $10 \mathrm{ml}$ TBST Buffer with gentle rocking the membranes were developed using Immobilon Crescendo Western HRP Substrate (Millipore, WBLUR0500) and imaged on an Odyssey Fc imaging system (LI-COR, Model: 2800). Buffer compositions and antibodies that were used and their concentrations are listed below. For histone western blots cells were trypsinized, resuspended in growth medium and pelleted by centrifugation. The pellet was resuspended in DPBS and cells were counted with an automated cell counter (Thermo Fisher Scientific, Countess II FL Automated Cell Counter). Equal numbers of cells were pelleted, resuspended in $1 \mathrm{x}$ SDS Laemmli Buffer, boiled for 10 minutes at $95^{\circ} \mathrm{C}$ on a heating block and centrifuged in a table top centrifuge for 5 minutes at full speed, RT. From the supernatant equal volumes (usually 5-15 $\mu \mathrm{l}$ ) were loaded and separated on a 4-20\% gradient SDSPAGE gel. The remainder of the workflow was identical to the one for the RIPA buffer extracted samples described above.

Antibody dilutions for western blot applications:

Mouse $\alpha$-Actin (DSHB, JLA20); 1:1,000 (supernatant). Mouse $\alpha$-DNTTIP1 (Novus Biologicals, NBP2-02507); 1:500. Rabbit $\alpha$-DNTTIP1 (Bethyl Laboratories, A304-048A); 1:2,000. Rabbit $\alpha$ DAB1 (Cell Signaling Technology, 3328); 1:5,000. Rabbit $\alpha$-pDAB1 (Cell Signaling Technology, 3327); 1:1,000. Rabbit $\alpha$-ELMSAN1 (This paper, 34421); 1:5,000. $\alpha$-FAK (Thermo Fisher Scientific, 39-6500); 1:1,000. $\alpha$-pFAK (Thermo Fisher Scientific, 700255); Rabbit $\alpha$-H3 (Abcam, ab1791); 1:50,000. Rabbit $\alpha-H 3 K 4 a c$ (RevMAb, 31-1063-00); 1:1,000. Rabbit $\alpha-H 3 K 27 a c$ (RevMAb, 31-1056-00); 1:1,000. Rabbit $\alpha-H 3$ K79ac (RevMAb, 31-1052-00); 1:1,000. Rabbit $\alpha$ H4 (Abcam, ab10158); 1:50,000. Rabbit $\alpha$-H4K20ac (RevMAb, 31-1084-00); 1:1,000. 1:1,000. Rabbit $\alpha$-HDAC1 (Cell Signaling Technology, 34589); 1:2,000. Rabbit $\alpha$-HDAC2 (Cell Signaling Technology, 57156); 1:2,000. Rabbit $\alpha$-MASH1 (Abcam, ab74065); 1:5,000. Sheep $\alpha$-NETRIN1 
(R\&D Systems, AF6419); 1:1,000. Mouse $\alpha$-PAX6 (DSHB, AB_528427); 1:500. Goat $\alpha$-ROBO3 (R\&D Systems, AF3155); 1:500. Rabbit $\alpha$-SLIT3 (Abcam, ab186706); 1:1,000. Mouse $\alpha$-UNC5B (R\&D Systems, AF6419); 1:1,000.

RIPA Buffer:

$25 \mathrm{mM}$ Tris $\mathrm{pH}$ 7.5; $150 \mathrm{mM} \mathrm{NaCl} ; 1 \mathrm{mM}$ EDTA; 1\% Triton X-100; 0.1\% SDS; 0.1\% Nadeoxycholate; protease inhibitors (Sigma, P8340) (1:100).

SDS-PAGE Running Buffer:

$25 \mathrm{mM}$ Tris $\mathrm{pH} 8.3,192 \mathrm{mM}$ glycine, $0.1 \%$ SDS.

Western Transfer Buffer:

$25 \mathrm{mM}$ Tris $\mathrm{pH} 8.3,192 \mathrm{mM}$ glycine, 20\% methanol.

TBST Buffer:

$20 \mathrm{mM}$ Tris pH 7.5, $150 \mathrm{mM} \mathrm{NaCl}, 0.1 \%$ Tween 20 .

4 x SDS Laemmli Buffer Stock:

$250 \mathrm{mM}$ Tris pH 6.8, 50\% Glycerol, 8\% SDS, 0.008\% Bromophenol blue

1 x SDS Laemmli Buffer:

$900 \mu 14$ X SDS Laemmli Buffer Stock $+100 \mu 1 \beta$-Mercaptoethanol

\section{CRISPR/Cas9 Genome Editing}

Genetically modified C57/BL6 Bruce-4 mESCs were generated using CRISPR/Cas9 technology. Briefly, 400,000 C57/BL6 Bruce-4 mESCs grown in feeder free culture conditions (DMEM KO medium) were transiently co-transfected with $500 \mathrm{ng}$ of gRNA expression plasmid (Addgene, 43860), $1 \mu \mathrm{g}$ Cas9 expression plasmid (Addgene, 43945), and $200 \mathrm{ng}$ of pMaxGFP via nucleofection (Lonza, 4D-Nucleofector ${ }^{\mathrm{TM}} \mathrm{X}$-unit) using solution P3 and program CA137 in small $(20 \mu \mathrm{l})$ cuvettes according to the manufacturer's recommended protocol. Cells were single cell sorted by FACS to enrich for GFP-positive (transfected) cells, clonally selected and verified for 
the desired targeted modification via targeted deep sequencing. Three clones were identified for each modification, assessed in relevant assays and then transitioned to chemically defined naïve culture conditions (2iL). Two clones for each gene knock-out were further used for more specific assays in this paper. The sequences for each gRNA and relevant primers are listed in Table S1.

\section{Quantification and Statistical Analysis}

\section{ChIP-seq analysis}

\section{Mapping reads and visualizing data}

ChIP-seq raw reads were aligned to the mouse and Drosophila melanogaster hybrid reference genomes $(\mathrm{mm} 9+\mathrm{dm} 3)$ using BWA (version 0.7.12; default parameter) and duplicated reads were then marked with Picard (version 1.65), with only nonduplicated reads kept by samtools (version 1.3.1, parameter "-q 1 -F 1024"'). Mapped reads were then split into two bam files (mapped to $\mathrm{mm} 9$ and $\mathrm{dm} 3$ respectively). For data quality control and to estimate the fragment size, the nonduplicated version of SPP (version 1.11) was used to calculate the relative strand correlation value with support of $\mathrm{R}$ (version 3.3.1). To visualize ChIP-seq data on the integrated genome viewer (IGV) (version 2.3.82), we utilized genomeCoverageBed (bedtools 2.25.0) to obtain genome-wide coverage in BEDGRAPH file format and then converted it to bigwig file format by bedGraphToBigWig. The bigwig files were scaled to 15 million reads to allow comparison across samples.

\section{Peak calling, annotation and motif analysis}

MACS2 (version 2.1.1 20160309) was used to call narrow peaks (DNTTIP1, HDAC1, H3K27ac and H3K4me3) with option "nomodel" and "extsize" defined as fragment size estimated by SPP and a FDR corrected p-value cutoff of 0.05 . For broad peak/domain calling (H3K4me1 and H3K27me3). SICER (version 1.1, with parameters of redundancy threshold 1, window size 200 bp, effective genome fraction 0.86, gap size $600 \mathrm{bp}$, FDR 0.00001 with fragment size defined above) was used for broad peak/domain calling (H3K4me1 and H3K27me3). Enriched regions were identified by comparing the ChIP library file to input library file. Peak regions were defined to be the union of peak intervals from two ChIP replicates of WT, Dnttipl KO or Elmsan1 KO mESCs, respectively. Promoter regions were defined as $\pm 1000 \mathrm{bp}$ from a TSS based on the mouse RefSeq annotation. Genomic feature annotation of peaks was carried out by annotatePeaks.pl, a 
program from HOMER suite (v4.8.3, http://homer.salk.edu/homer/). HOMER software was used to perform de novo motif discovery and to check for enrichment of known motifs from a set of DNTTIP1 peaks (associated with up- or downregulated genes in Dnttipl KO versus WT mESCs) or all DNTTIP1 peaks.

\section{Spike-in normalization and differential analysis}

ChIP-seq raw read counts were reported for each region/each sample using bedtools 2.25.0. Spikein normalization was performed by counting Drosophila reads and mouse reads in each ChIP sample and corresponding input sample and using those counts to generate a normalization factor for each sample, which was calculated as (ChIP_dm3.reads/ChIP_mm9.reads)/(Input_dm3.reads/Input_mm9.reads). Raw read counts were voom normalized and statistically contrasted using the pipeline limma in R (version 3.3.1). The normalization factor defined above was used to modify the mouse library size in edgeR (version 3.16.5) for CPM calculation and differential analysis. An empirical Bayes fit was applied to contrast Dnttip1 KO and Elmsan1 KO samples to WT samples and to generate log2 fold changes, p-values and false discovery rates for each peak region. Histograms showing average ChIP-seq intensity over gene bodies were generated using ngsplot (v2.61).

\section{$\underline{\text { RNA-seq analysis }}$}

Total stranded RNA sequencing data were generated and mapped against mouse genome assembly NCBIM37.67 using the StrongArm pipeline described previously (Wu, Barnhill et al., 2016). Gene level quantification values were obtained with HT-seq based on the GENCODE annotation (vM20) and normalized by the TMM method with edgeR (version 3.16.5). Differential expression analysis was performed with the voom method applying the limma pipeline in $\mathrm{R}$ (version 3.3.1). Significantly up- and down- regulated genes were defined by at least a 1.5 fold change in gene expression and a p-value $<0.01$. Reactome and gene set enrichment analysis (GSEA) were carried out using GSEA or EnrichR, respectively. Gene expression $\log _{2} \mathrm{CPM}$ (counts per million) values were computed for heatmap and box plot visualization. Log2 FPKM gene expression values were applied for bar plot diagrams.

\section{$\underline{\text { Neurite and neuron analysis }}$}


The neuron-specific markers TUBB3 and MAP2 were used to identify neurons by IF as described under "Neural Differentiation". NeuriteTracer, a plugin of ImageJ (version 1.52a), was used to manually trace the length and number of neurites per neuron and to automatically detect nuclei based on DAPI staining using the IF raw image data for each genotype. The length of neurites was determined from 200 neurons for each genotype. The number of neurites per neuron was determined manually for a total of 200 neurons per genotype. The percentage of neurons within the total cell population in NE was calculated manually by determining the number of MAP2positive neurons in relation to the total number of cells as determined by DAPI staining or alternatively by flow cytometry analysis of TUBB3/DAPI-stained NE cell populations. Neurite length was classified into shorter neurites $(<50 \mu \mathrm{m})$ and longer neurites $(\geq 50 \mu \mathrm{m}$; possibly axons) and the mean length was compared amongst relevant conditions by applying the Student's t-test. $\%$ of neurons $=($ number of neurons per image as assessed by MAP2 staining/total number of cells per image as assessed by DAPI staining) x 100. For each analysis, experiments were carried out in triplicate. The number of analyzed neurons are indicated in the relevant figure legends. An unpaired Student's t-test as well as ONE-way ANOVA was performed for each of the treated conditions and compared either to the WT or to their respective controls.

Statistical analysis of qPCR and qRT-PCR data qPCR signals for manual ChIPs were calculated as \% of input (\% Input) from technical duplicates. Error bars represent the standard deviation from technical duplicates. For qRT-PCR relative gene expression levels were calculated applying the delta-delta $\mathrm{Ct}$ method $\left(2^{-\Delta \Delta \mathrm{Ct}}\right)$. Error bars depict the standard deviation from technical triplicates. Statistical significance was determined by applying the Student's unpaired t-test.

\section{Public datasets and additional bioinformatics analysis}

HDAC1 ChIP-seq data from WT mESCs was obtained from gene expression omnibus (GEO): GSM1335482. Data was analyzed as described under "Quantification and statistical analysis" within the "ChIP-seq analysis" section if applicable. 
bioRxiv preprint doi: https://doi.org/10.1101/2020.04.07.029751; this version posted April 7, 2020. The copyright holder for this preprint (which was not certified by peer review) is the author/funder, who has granted bioRxiv a license to display the preprint in perpetuity. It is made available under aCC-BY 4.0 International license.

\section{Data availability}

The accession number for the RNA-seq and ChIP-seq datasets reported in this paper is GEO: GSE131062. 


\section{Acknowledgements}

We thank BaoHan Vo and Martine Roussel for generously providing granule neuron progenitor cells; Alyssa House, Scott Perry, Mohona Sarkar, and Richard Ashmun from the Flow Cytometry and Cell Sorting Shared Resource for conducting cytometry and FACS analyses; Dana Roeber, Rain Sun, Sanchit Trivedi, Scott Olsen, and Geoffrey Neale from the Hartwell Center for RNAand ChIP-seq library preparation, sequencing, and support; and Jamshid Temirov for advice and guidance with microscopy-related applications. We thank all Herz lab members for insightful comments and critical reading of the manuscript. This work was supported by a transition to independence grant from the National Institutes of Health/National Cancer Institute (R00CA181506) to H.-M.H and the American Lebanese Syrian Associated Charities (ALSAC). 


\section{Author contributions}

H.-M.H. and B.M. conceived, designed, conducted the studies, performed experiments, and wrote the manuscript. H.J. and Y.F. provided bioinformatics support. S.K., Y.S., and T.M. assisted in experiments. M.F.S. and S.M.P.-M. generated knockout mESCs. C.L. provided active mentorship and guidance in culturing and differentiation of mESCs. GJP aided in manuscript writing. 
bioRxiv preprint doi: https://doi.org/10.1101/2020.04.07.029751; this version posted April 7, 2020. The copyright holder for this preprint (which was not certified by peer review) is the author/funder, who has granted bioRxiv a license to display the preprint in perpetuity. It is made available under aCC-BY 4.0 International license.

\section{Conflict of interest}

The authors declare that they have no conflict of interest. 


\section{References}

Alsina FC, Irala D, Fontanet PA, Hita FJ, Ledda F, Paratcha G (2012) Sprouty4 is an endogenous negative modulator of TrkA signaling and neuronal differentiation induced by NGF. PloS one 7: e32087

Andres ME, Burger C, Peral-Rubio MJ, Battaglioli E, Anderson ME, Grimes J, Dallman J, Ballas N, Mandel G (1999) CoREST: a functional corepressor required for regulation of neural-specific gene expression. Proceedings of the National Academy of Sciences of the United States of America 96: 9873-8

Banks CAS, Miah S, Adams MK, Eubanks CG, Thornton JL, Florens L, Washburn MP (2018) Differential HDAC1/2 network analysis reveals a role for prefoldin/CCT in HDAC1/2 complex assembly. Sci Rep 8: 13712

Bantscheff M, Hopf C, Savitski MM, Dittmann A, Grandi P, Michon AM, Schlegl J, Abraham Y, Becher I, Bergamini G, Boesche M, Delling M, Dumpelfeld B, Eberhard D, Huthmacher C, Mathieson T, Poeckel D, Reader V, Strunk K, Sweetman G et al. (2011) Chemoproteomics profiling of HDAC inhibitors reveals selective targeting of HDAC complexes. Nat Biotechnol 29: $255-65$

Bashaw GJ, Klein R (2010) Signaling from axon guidance receptors. Cold Spring Harb Perspect Biol 2: a001941

Bernstein BE, Tong JK, Schreiber SL (2000) Genomewide studies of histone deacetylase function in yeast. Proceedings of the National Academy of Sciences of the United States of America 97: $13708-13$

Besnard A, Galan-Rodriguez B, Vanhoutte P, Caboche J (2011) Elk-1 a transcription factor with multiple facets in the brain. Front Neurosci 5: 35

Blockus H, Chedotal A (2016) Slit-Robo signaling. Development 143: 3037-44

Chen Y, Wang H, Yoon SO, Xu X, Hottiger MO, Svaren J, Nave KA, Kim HA, Olson EN, Lu QR (2011) HDAC-mediated deacetylation of NF-kappaB is critical for Schwann cell myelination. Nature neuroscience 14: 437-41 
Choi E, Han C, Park I, Lee B, Jin S, Choi H, Kim DH, Park ZY, Eddy EM, Cho C (2008) A novel germ cell-specific protein, SHIP1, forms a complex with chromatin remodeling activity during spermatogenesis. J Biol Chem 283: 35283-94

Dahl JA, Collas P (2008) A rapid micro chromatin immunoprecipitation assay (microChIP). Nat Protoc 3: 1032-45

Doetsch F, Alvarez-Buylla A (1996) Network of tangential pathways for neuronal migration in adult mammalian brain. Proceedings of the National Academy of Sciences of the United States of America 93: 14895-900

Gehman LT, Meera P, Stoilov P, Shiue L, O'Brien JE, Meisler MH, Ares M, Jr., Otis TS, Black DL (2012) The splicing regulator Rbfox 2 is required for both cerebellar development and mature motor function. Genes Dev 26: 445-60

Gilbert J, Man HY (2017) Fundamental Elements in Autism: From Neurogenesis and Neurite Growth to Synaptic Plasticity. Front Cell Neurosci 11: 359

Gizard F, Lavallee B, DeWitte F, Hum DW (2001) A novel zinc finger protein TReP-132 interacts with CBP/p300 to regulate human CYP11A1 gene expression. J Biol Chem 276: 33881-92

Gizard F, Lavallee B, DeWitte F, Teissier E, Staels B, Hum DW (2002) The transcriptional regulating protein of $132 \mathrm{kDa}$ (TReP-132) enhances P450scc gene transcription through interaction with steroidogenic factor-1 in human adrenal cells. J Biol Chem 277: 39144-55

Gizard F, Robillard R, Barbier O, Quatannens B, Faucompre A, Revillion F, Peyrat JP, Staels B, Hum DW (2005) TReP-132 controls cell proliferation by regulating the expression of the cyclindependent kinase inhibitors p21WAF1/Cip1 and p27Kip1. Mol Cell Biol 25: 4335-48

Gizard F, Robillard R, Gross B, Barbier O, Revillion F, Peyrat JP, Torpier G, Hum DW, Staels B (2006) TReP-132 is a novel progesterone receptor coactivator required for the inhibition of breast cancer cell growth and enhancement of differentiation by progesterone. Mol Cell Biol 26: 763244 
Gizard F, Teissier E, Dufort I, Luc G, Luu-The V, Staels B, Hum DW (2004) The transcriptional regulating protein of $132 \mathrm{kDa}$ (TReP-132) differentially influences steroidogenic pathways in human adrenal NCI-H295 cells. J Mol Endocrinol 32: 557-69

Graff J, Rei D, Guan JS, Wang WY, Seo J, Hennig KM, Nieland TJ, Fass DM, Kao PF, Kahn M, Su SC, Samiei A, Joseph N, Haggarty SJ, Delalle I, Tsai LH (2012) An epigenetic blockade of cognitive functions in the neurodegenerating brain. Nature 483: 222-6

Guan JS, Haggarty SJ, Giacometti E, Dannenberg JH, Joseph N, Gao J, Nieland TJ, Zhou Y, Wang X, Mazitschek R, Bradner JE, DePinho RA, Jaenisch R, Tsai LH (2009) HDAC2 negatively regulates memory formation and synaptic plasticity. Nature 459: 55-60

Hao Y, Xu N, Box AC, Schaefer L, Kannan K, Zhang Y, Florens L, Seidel C, Washburn MP, Wiegraebe W, Mak HY (2011) Nuclear cGMP-dependent kinase regulates gene expression via activity-dependent recruitment of a conserved histone deacetylase complex. PLoS Genet 7: e1002065

Harrison MR, Georgiou AS, Spaink HP, Cunliffe VT (2011) The epigenetic regulator Histone Deacetylase 1 promotes transcription of a core neurogenic programme in zebrafish embryos. BMC Genomics 12: 24

Hein MY, Hubner NC, Poser I, Cox J, Nagaraj N, Toyoda Y, Gak IA, Weisswange I, Mansfeld J, Buchholz F, Hyman AA, Mann M (2015) A human interactome in three quantitative dimensions organized by stoichiometries and abundances. Cell 163: 712-23

Huang XF, Song X (2019) Effects of antipsychotic drugs on neurites relevant to schizophrenia treatment. Med Res Rev 39: 386-403

Huttlin EL, Ting L, Bruckner RJ, Gebreab F, Gygi MP, Szpyt J, Tam S, Zarraga G, Colby G, Baltier K, Dong R, Guarani V, Vaites LP, Ordureau A, Rad R, Erickson BK, Wuhr M, Chick J, Zhai B, Kolippakkam D et al. (2015) The BioPlex Network: A Systematic Exploration of the Human Interactome. Cell 162: 425-440

Itoh T, Fairall L, Muskett FW, Milano CP, Watson PJ, Arnaudo N, Saleh A, Millard CJ, ElMezgueldi M, Martino F, Schwabe JW (2015) Structural and functional characterization of a cell 
cycle associated HDAC1/2 complex reveals the structural basis for complex assembly and nucleosome targeting. Nucleic Acids Res 43: 2033-44

Jacob C, Christen CN, Pereira JA, Somandin C, Baggiolini A, Lotscher P, Ozcelik M, Tricaud N, Meijer D, Yamaguchi T, Matthias P, Suter U (2011) HDAC1 and HDAC2 control the transcriptional program of myelination and the survival of Schwann cells. Nature neuroscience 14: 429-36

Ji X, Dadon DB, Abraham BJ, Lee TI, Jaenisch R, Bradner JE, Young RA (2015) Chromatin proteomic profiling reveals novel proteins associated with histone-marked genomic regions. Proceedings of the National Academy of Sciences of the United States of America 112: 3841-6

Joshi P, Greco TM, Guise AJ, Luo Y, Yu F, Nesvizhskii AI, Cristea IM (2013) The functional interactome landscape of the human histone deacetylase family. Mol Syst Biol 9: 672

Kadosh D, Struhl K (1998) Histone deacetylase activity of Rpd3 is important for transcriptional repression in vivo. Genes Dev 12: 797-805

Kaimori JY, Maehara K, Hayashi-Takanaka Y, Harada A, Fukuda M, Yamamoto S, Ichimaru N, Umehara T, Yokoyama S, Matsuda R, Ikura T, Nagao K, Obuse C, Nozaki N, Takahara S, Takao T, Ohkawa Y, Kimura H, Isaka Y (2016) Histone H4 lysine 20 acetylation is associated with gene repression in human cells. Sci Rep 6: 24318

Kelly RD, Cowley SM (2013) The physiological roles of histone deacetylase (HDAC) 1 and 2: complex co-stars with multiple leading parts. Biochem Soc Trans 41: 741-9

Kim JY, Shen S, Dietz K, He Y, Howell O, Reynolds R, Casaccia P (2010) HDAC1 nuclear export induced by pathological conditions is essential for the onset of axonal damage. Nature neuroscience 13: 180-9

Kim KT, Kim N, Kim HK, Lee H, Gruner HN, Gergics P, Park C, Mastick GS, Park HC, Song MR (2016) ISL1-based LIM complexes control Slit2 transcription in developing cranial motor neurons. Sci Rep 6: 36491 
Knock E, Pereira J, Lombard PD, Dimond A, Leaford D, Livesey FJ, Hendrich B (2015) The methyl binding domain 3/nucleosome remodelling and deacetylase complex regulates neural cell fate determination and terminal differentiation in the cerebral cortex. Neural Dev 10: 13

Koiwai K, Kubota T, Watanabe N, Hori K, Koiwai O, Masai H (2015) Definition of the transcription factor TdIF1 consensus-binding sequence through genomewide mapping of its binding sites. Genes Cells 20: 242-54

Krejci J, Legartova S, Bartova E (2017) Neural Differentiation in HDAC1-Depleted Cells Is Accompanied by Coilin Downregulation and the Accumulation of Cajal Bodies in Nucleoli. Stem Cells Int 2017: 1021240

Kubota T, Koiwai O, Hori K, Watanabe N, Koiwai K (2013) TdIF1 recognizes a specific DNA sequence through its Helix-Turn-Helix and AT-hook motifs to regulate gene transcription. PloS one 8: e66710

Labrador JP, O'Keefe D, Yoshikawa S, McKinnon RD, Thomas JB, Bashaw GJ (2005) The homeobox transcription factor even-skipped regulates netrin-receptor expression to control dorsal motor-axon projections in Drosophila. Curr Biol 15: 1413-9

Lai Wing Sun K, Correia JP, Kennedy TE (2011) Netrins: versatile extracellular cues with diverse functions. Development 138: 2153-69

Lee TI, Johnstone SE, Young RA (2006) Chromatin immunoprecipitation and microarray-based analysis of protein location. Nature protocols 1: 729-48

Li C, Yu H, Ma Y, Shi G, Jiang J, Gu J, Yang Y, Jin S, Wei Z, Jiang H, Li J, Jin Y (2009) Germlinecompetent mouse-induced pluripotent stem cell lines generated on human fibroblasts without exogenous leukemia inhibitory factor. PloS one 4: e6724

Livak KJ, Schmittgen TD (2001) Analysis of relative gene expression data using real-time quantitative PCR and the 2(-Delta Delta C(T)) Method. Methods 25: 402-8 
Lyden D, Young AZ, Zagzag D, Yan W, Gerald W, O'Reilly R, Bader BL, Hynes RO, Zhuang Y, Manova K, Benezra R (1999) Id1 and Id3 are required for neurogenesis, angiogenesis and vascularization of tumour xenografts. Nature 401: 670-7

Millard CJ, Watson PJ, Fairall L, Schwabe JWR (2017) Targeting Class I Histone Deacetylases in a "Complex" Environment. Trends Pharmacol Sci 38: 363-377

Montgomery RL, Hsieh J, Barbosa AC, Richardson JA, Olson EN (2009) Histone deacetylases 1 and 2 control the progression of neural precursors to neurons during brain development. Proceedings of the National Academy of Sciences of the United States of America 106: 7876-81

Nam HS, Benezra R (2009) High levels of Id1 expression define B1 type adult neural stem cells. Cell Stem Cell 5: 515-26

Nitarska J, Smith JG, Sherlock WT, Hillege MM, Nott A, Barshop WD, Vashisht AA, Wohlschlegel JA, Mitter R, Riccio A (2016) A Functional Switch of NuRD Chromatin Remodeling Complex Subunits Regulates Mouse Cortical Development. Cell Rep 17: 1683-1698

Pagliuca FW, Collins MO, Lichawska A, Zegerman P, Choudhary JS, Pines J (2011) Quantitative proteomics reveals the basis for the biochemical specificity of the cell-cycle machinery. Molecular cell 43: 406-17

Rundlett SE, Carmen AA, Suka N, Turner BM, Grunstein M (1998) Transcriptional repression by UME6 involves deacetylation of lysine 5 of histone H4 by RPD3. Nature 392: 831-5

Sawai Y, Kasamatsu A, Nakashima D, Fushimi K, Kasama H, Iyoda M, Kouzu Y, Shiiba M, Tanzawa H, Uzawa K (2018) Critical role of deoxynucleotidyl transferase terminal interacting protein 1 in oral cancer. Lab Invest

Seiradake E, Jones EY, Klein R (2016) Structural Perspectives on Axon Guidance. Annu Rev Cell Dev Biol 32: 577-608

Shahbazian MD, Grunstein M (2007) Functions of site-specific histone acetylation and deacetylation. Annual review of biochemistry 76: 75-100 
Shepherd GM (1998) The synaptic organization of the brain. Oxford University Press, New York

Vo BT, Wolf E, Kawauchi D, Gebhardt A, Rehg JE, Finkelstein D, Walz S, Murphy BL, Youn YH, Han YG, Eilers M, Roussel MF (2016) The Interaction of Myc with Miz1 Defines Medulloblastoma Subgroup Identity. Cancer Cell 29: 5-16

Wang Y, Wu Q, Yang P, Wang C, Liu J, Ding W, Liu W, Bai Y, Yang Y, Wang H, Gao S, Wang X (2016) LSD1 co-repressor Rcor2 orchestrates neurogenesis in the developing mouse brain. Nat Commun 7: 10481

Wang Z, Zang C, Cui K, Schones DE, Barski A, Peng W, Zhao K (2009) Genome-wide mapping of HATs and HDACs reveals distinct functions in active and inactive genes. Cell 138: 1019-31

Wen Z, Cheng TL, Li GZ, Sun SB, Yu SY, Zhang Y, Du YS, Qiu Z (2017) Identification of autismrelated MECP2 mutations by whole-exome sequencing and functional validation. Mol Autism 8: 43

Wu G, Barnhill RL, Lee S, Li Y, Shao Y, Easton J, Dalton J, Zhang J, Pappo A, Bahrami A (2016) The landscape of fusion transcripts in spitzoid melanoma and biologically indeterminate spitzoid tumors by RNA sequencing. Mod Pathol 29: 359-69

Yamaguchi T, Cubizolles F, Zhang Y, Reichert N, Kohler H, Seiser C, Matthias P (2010) Histone deacetylases 1 and 2 act in concert to promote the G1-to-S progression. Genes Dev 24: 455-69

Yang WM, Inouye C, Zeng Y, Bearss D, Seto E (1996) Transcriptional repression by YY1 is mediated by interaction with a mammalian homolog of the yeast global regulator RPD3. Proceedings of the National Academy of Sciences of the United States of America 93: 12845-50

Yang WM, Yao YL, Sun JM, Davie JR, Seto E (1997) Isolation and characterization of cDNAs corresponding to an additional member of the human histone deacetylase gene family. J Biol Chem 272: $28001-7$

Ye F, Chen Y, Hoang T, Montgomery RL, Zhao XH, Bu H, Hu T, Taketo MM, van Es JH, Clevers H, Hsieh J, Bassel-Duby R, Olson EN, Lu QR (2009) HDAC1 and HDAC2 regulate 
oligodendrocyte differentiation by disrupting the beta-catenin-TCF interaction. Nat Neurosci 12 : 829-38

Zelina P, Blockus H, Zagar Y, Peres A, Friocourt F, Wu Z, Rama N, Fouquet C, Hohenester E, Tessier-Lavigne M, Schweitzer J, Roest Crollius H, Chedotal A (2014) Signaling switch of the axon guidance receptor Robo3 during vertebrate evolution. Neuron 84: 1258-72

Zhang Y, Wang Z, Huang Y, Ying M, Wang Y, Xiong J, Liu Q, Cao F, Joshi R, Liu Y, Xu D, Zhang M, Yuan K, Zhou N, Koropatnick J, Min W (2018) TdIF1: a putative oncogene in NSCLC tumor progression. Signal Transduct Target Ther 3: 28

Zupkovitz G, Tischler J, Posch M, Sadzak I, Ramsauer K, Egger G, Grausenburger R, Schweifer N, Chiocca S, Decker T, Seiser C (2006) Negative and positive regulation of gene expression by mouse histone deacetylase 1. Mol Cell Biol 26: 7913-28 


\section{Figure Legends}

\section{Figure 1. MiDAC controls a neurodevelopmental gene expression program.}

A Subunits with domains of the human histone deacetylase complex MiDAC.

B WB for the indicated MiDAC components from total cell lysates of WT, Dnttip1 KO1 and Dnttipl KO2 mESCs. Actin is the loading control.

C IPs were carried out with IgG and ELMSAN1 antibodies from nuclear extracts of WT and Dnttip1 KO1 mESCs followed by WB for the indicated MiDAC components. The asterisk marks the IgG heavy chain.

D Scatter plot comparing all DEGs in Dnttip1 KO (KO1 and KO2) versus WT mESCs from Figure S2A (x-axis) with DEGs in Elmsan1 KO (KO1 and KO2) versus WT mESCs from Figure S2B (yaxis). Both axes depict normalized gene expression ( $\log _{2} \mathrm{FC}$ of CPM).

E RNA-seq heatmap depicting DNTTIP1 and ELMSAN1 (MiDAC) co-regulated genes in mESCs (fold change $(\mathrm{FC})>1.5$ or $<-1.5, \mathrm{p}<0.01$ ). The color scale depicts normalized gene expression $\left(\log _{2} \mathrm{CPM}\right)$.

F Reactome analysis showing the most highly enriched gene categories of genes that are positively regulated by MiDAC (both down in Dnttip1 KO (KO1 and KO2) and Elmsan1 KO (KO1 and KO2) versus WT mESCs, FC $<-1.5$ or $>1.5, \mathrm{p}<0.01$ ). Pathways associated with neural differentiation and function are highlighted in red.

G RNA-seq heatmaps depicting down- and upregulated genes from a gene set of neurodevelopmental genes that is mutually regulated by DNTTIP1 and ELMSAN1 $(\mathrm{FC}<-1.5$ or $>1.5, \mathrm{p}<0.05)$. The color scale depicts the $\mathrm{z}$-score of normalized gene expression $\left(\log _{2} \mathrm{CPM}\right)$.

\section{Figure 2. MiDAC regulates neurite outgrowth.}

A Schematic outline of neuro-ectoderm (NE) differentiation protocol.

B MAP2 immunofluorescence (IF) staining of WT, Dnttip1 KO1 and Elmsan1 KO1 NE after 12 days of differentiation. Nuclei were stained with DAPI. For analysis the neuronal cell body (blue) and its neurites were manually traced with ImageJ software. The white scale bar represents $20 \mu \mathrm{m}$. C, D qRT-PCR for C Tubb3 and D Map2 mRNA in WT, Dnttip1 KO1 and Elmsan1 KO1 mESCs (day 0 ) and NE after 12 and 21 days of differentiation. Expression was normalized to Gapdh. E-G Quantification of $\mathbf{E}$ neurite length, $\mathbf{F}$ the total number of neurites per neuron and $\mathbf{G}$ the percentage of neurons within the total cell population from traced neurites in WT, Dnttip1 KO1 
and Elmsan1 KO1 neurons after 12 days of differentiation as determined by MAP2 IF. E, $\mathbf{F}$ The neurites of 200 neurons within the total cell population were assessed per genotype.

H Gene expression levels of the indicated neurodevelopmental genes in WT, Dnttipl KO1 and Elmsan1 KO1 NE after 12 days of differentiation as analyzed by qRT-PCR. Expression was normalized to Gapdh.

Unpaired t-test was performed throughout where ***, $\mathrm{p} \leq 0.001 ; * *, \mathrm{p} \leq 0.01 ; *, \mathrm{p} \leq 0.05$; and ns, $\mathrm{p}>0.05$ is not significant.

Figure 3. MiDAC binds to and modulates the expression of genes that regulate neural differentiation and neurite outgrowth.

A Pie charts displaying the genome-wide distribution (left) and promoter distal distribution (right) of DNTTIP1 in WT mESCs. DNTTIP1 peaks within $1 \mathrm{~kb}$ of the transcription start site (TSS) were assigned to TSS.

B Venn Diagram depicting the overlap between DNTTIP1, H3K4me1 and H3K27ac peaks in WT mESCs.

C Venn diagram showing the number of downregulated and upregulated genes that are bound by DNTTIP1 in WT mESCs.

D Heatmaps displaying the genome-wide distribution of all DNTTIP1 binding sites in WT, Dnttip1 KO1 and Elmsan1 KO1 mESCs sorted by enrichment in descending order in WT mESCs and compared to HDAC1 occupancy in WT mESCs. The color scale depicts the normalized ChIP-seq signal intensity ( $\log _{2} \mathrm{CPM}$ per $20 \mathrm{bp}$ bin).

E Venn diagram showing the co-occupancy between DNTTIP1 and HDAC1 peaks in WT mESCs. F, G ChIP-seq profiles of the F Slit3 and G Spry4 loci for DNTTIP1 in WT, Dnttip1 KO1 and Elmsan1 KO1 mESCs and for HDAC1 in WT mESCs. Promoter and putative enhancer regions used for manual ChIP experiments in Figure 4 and Figure S6 are highlighted by orange boxes.

H WB for the specified histone acetylation marks from total cell lysates of WT, Dnttip1 KO1 and Elmsan1 KO1 mESCs. H3 and H4 are loading controls.

Figure 4. MiDAC directly targets positive and negative regulators of neurite outgrowth during neural differentiation. 
A-J qPCR from manual ChIP experiments against A, F DNTTIP1, B, G ELMSAN1, C, H HDAC1, D, I H3K27ac and E, J H4K20ac from WT, Dnttipl KO1 and Elmsanl KO1 NE targeting select promoter, putative enhancer and intragenic control regions of A-D Slit3 or E-H Spry4 loci as highlighted in Figure 3F and G. IgG was used as a control antibody. Unpaired t-test was performed throughout where ${ }^{* *}, \mathrm{p} \leq 0.01$; and $\mathrm{ns}, \mathrm{p}>0.05$ is not significant.

Figure 5. The regulatory role of MiDAC in neural differentiation and neurite outgrowth is carried out in part by secreted components.

A MAP2 IF staining after 12 days of differentiation performed on WT, Dnttip1 KO1 and Elmsan1 KO1 NE supplemented daily with conditioned medium (CM) of WT NE from day 7-12. Nuclei were stained with DAPI. For analysis the neuronal cell body (blue) and its neurites (black) were manually traced with ImageJ software and for each sample one traced neuron is displayed in the inlet. The white scale bar represents $50 \mu \mathrm{m}$.

B, C Quantification of $\mathbf{B}$ neurite length and $\mathbf{C}$ the total number of neurites per neuron from the MAP2 IF staining in Figure 5A using ImageJ. B Neurite length was divided into two categories of short neurites $<50 \mu \mathrm{m}$ (green box plots) and longer neurites $\geq 50 \mu \mathrm{m}$ (white box plots). B, $\mathbf{C}$ The neurites of 200 neurons were assessed per sample.

One-way ANOVA was performed throughout where ***, $\mathrm{p} \leq 0.001 ; * *, \mathrm{p} \leq 0.01$; and ${ }^{*}, \mathrm{p} \leq 0.05$.

Figure 6. MiDAC regulates neurite outgrowth via the SLIT3/ROBO3 and NTN1/UNC5B signaling pathways.

A WB for signaling components of the SLIT3/ROBO3 and NTN1/DCC/UNC5B signaling axes from CM and total cell lysates of WT and Dnttipl KO1 NE after 12 days of differentiation. To enrich SLIT3 and NTN1 from CM, IPs were performed with SLIT3 and NTN1 antibodies from CM of WT and Dnttipl KO1 NE. Actin is the loading control for the total cell lysates.

B Assay to rescue the neurite outgrowth defects in Dnttip1 KO1 NE. CM of Dnttip1 KO1 NE was supplemented with the recombinant signaling ligands SLIT3 and/or NTN1 from day 7-12 without or with preblocking of Dnttip1 KO1 NE with IgG or signaling receptor antibodies against ROBO3 and/or UNC5B. MAP2 IF staining was performed after 12 days of differentiation. Nuclei were stained with DAPI. To facilitate analysis the neuronal cell body (blue) and its neurites were 
manually traced with ImageJ software and for each sample one traced neuron is displayed in the inlet. The white scale bar represents $50 \mu \mathrm{m}$.

C, D Quantification of $\mathbf{C}$ neurite length and $\mathbf{D}$ the total number of neurites per neuron from the MAP2 IF staining in Fig. 6e using ImageJ. C Neurite length was divided into two categories of short neurites $<50 \mu \mathrm{m}$ (green box plots) and longer neurites $\geq 50 \mu \mathrm{m}$ (white box plots). C, D The neurites of 200 neurons were assessed per sample.

One-way ANOVA was performed throughout where $* * *, p \leq 0.001$; and $n s, p>0.05$ is not significant.

\section{Figure 7. Model of MiDAC function in neurite outgrowth and morphogenesis.}

MiDAC directly binds to regulatory elements of pro-neural genes such as those of the axon guidance ligands SLIT3 and NTN1 resulting in their activation while simultaneously inhibiting expression of genes of negative regulators of neurogenesis such as SPRY4 and ID1 by removing $\mathrm{H} 3 \mathrm{~K} 27 \mathrm{ac}$ from their promoters and enhancers. 


\section{Supplemental Information}

\section{Supplemental Figure Legends}

Figure S1. Characterization of Dnttip1 KO and Elmsan1 KO mESCs.

A, B Gene structures of Dnttipl and Elmsan1 highlighting the region within exon 2 that was targeted by CRISPR/Cas9 in mESCs. The gDNA sequence is shown underlined in red. The resulting indels for each allele within the A Dnttipl KO1, Dnttipl KO2 and B Elmsan1 KO1 and Elmsan1 KO2 clones are highlighted by red boxes.

C, D RNA-seq tracks of the C Dnttipl and D Elmsan1 locus in WT, Dnttip1 KO1 and Elmsanl KO1 mESCs.

E qRT-PCR for Dnttip1, Elmsan1, Hdac1, and Hdac2 mRNA in WT Dnttip1 KO (KO1 and KO2) and Elmsan1 KO (KO1 and KO2) mESCs. Expression was normalized to Gapdh.

F WB for the indicated MiDAC components from total cell lysates of WT, Elmsan1 KO1 and Elmsan1 KO2 mESCs. Actin is the loading control.

Unpaired t-test was performed throughout where **, $\mathrm{p} \leq 0.01$.

G IPs were carried out with IgG and DNTTIP1 antibodies from nuclear extracts of WT and Elmsan1 KO1 mESCs followed by WB for the indicated MiDAC components. The asterisk marks the IgG heavy chain.

Figure S2. MiDAC controls a neurodevelopmental gene expression program.

A, B RNA-seq heatmap obtained by hierarchical clustering of differentially expressed genes (DEGs) in A WT and Dnttipl KO (KO1 and KO2) and B WT and Elmsan1 KO (KO1 and KO2) mESCs (fold change $(\mathrm{FC})>1.5$ or $<-1.5, \mathrm{p}<0.01$ ). The color scale depicts normalized gene expression ( $\left.\log _{2} \mathrm{CPM}\right)$.

C Venn diagram showing the numbers of a downregulated (blue, 224 genes) and upregulated (red, 114 genes) gene set that is common to both Dnttip1 KO (KO1 and KO2) and Elmsan1 KO (KO1 and $\mathrm{KO} 2)$ mESCs $(\mathrm{FC}<-1.5$ or $>1.5, \mathrm{p}<0.01)$.

$\mathbf{D}, \mathbf{E}$ Reactome analysis showing the most highly enriched gene categories of $\mathbf{D}$ down- and $\mathbf{E}$ upregulated genes in Dnttip1 KO (KO1 and KO2) versus WT mESCs $(\mathrm{FC}<-1.5$ or $>1.5$, p < 0.01). Pathways associated with neuronal differentiation and function are highlighted in red. 
F, G Reactome analysis showing the most highly enriched gene categories of $\mathbf{F}$ down- and $\mathbf{G}$ upregulated genes in Elmsan1 $\mathrm{KO}(\mathrm{KO} 1$ and $\mathrm{KO} 2)$ versus WT mESCs $(\mathrm{FC}<-1.5$ or $>1.5, \mathrm{p}<$ 0.01). Pathways associated with neuronal differentiation and function are highlighted in red.

Figure S3. DNTTIP1 is dispensable for self-renewal, pluripotency and cell cycle distribution of mESCs.

A RNA-seq tracks of the Sox2, Pou5f1 and Nanog loci in WT, Dnttip1 KO1 and Elmsan1 KO1 mESCs.

B qRT-PCR for Sox2, Pou5f1 and Nanog mRNA in WT, Dnttipl KO1 and Elmsan1 KO1 mESCs. Expression was normalized to Gapdh.

C Alkaline phosphatase (AP) staining and quantification of AP positive WT, Dnttipl KO (KO1 and $\mathrm{KO} 2)$ and Elmsan1 $\mathrm{KO}(\mathrm{KO} 1$ and $\mathrm{KO} 2)$ mESC colonies as percentage of the whole population.

D Proliferation time course comparing WT with Dnttipl KO (KO1 and KO2) and WT with Elmsan1 KO (KO1 and KO2) mESCs.

E Cell cycle distribution of WT, Dnttipl KO (KO1 and KO2) and Elmsan1 KO (KO1 and KO2) mESCs. Black: G0/G1 phase. White: S phase. Gray: M phase.

F Box plots showing the normalized gene expression levels $\left(\log _{2} \mathrm{CPM}\right)$ of cyclin $\mathrm{A} 2$ (Ccna2) and the cyclin-dependent kinase inhibitors Cdkn1a (p21) and Cdkn1b (p27) in WT, Dnttip1 KO (KO1 and $\mathrm{KO} 2)$ and Elmsan1 KO (KO1 and KO2) mESCs.

Figure S4. Loss of MiDAC function does not alter the differentiation and cell cycle distribution of neural progenitor cells.

A, B qRT-PCR for A Nes and B Pax6 mRNA in WT, Dnttipl KO1 and Elmsan1 KO1 mESCs and NE after 8 days of differentiation. Expression was normalized to Gapdh.

C, D WB for C PAX6 and D MASH1 from total cell lysates of WT, Dnttip1 KO1 and Elmsan1 KO1 mESCs and NE after $\mathbf{C} 8$ days and D 8 and 12 days of differentiation. Actin is the loading control.

$\mathbf{E}, \mathbf{F}$ Cell cycle distribution of $\mathbf{E}$ PAX6 positive and F TUBB3 positive cells of WT, Dnttip1 KO (KO1 and KO2) and Elmsan1 KO (KO1 and KO2) NE after $\mathbf{E} 8$ and $\mathbf{F} 12$ days of differentiation. Black: G0/G1 phase. White: S phase. Gray: M phase. 
Figure S5. MiDAC binds to and modulates the expression of genes that regulate neural differentiation and neurite outgrowth.

A Heatmaps displaying the genome-wide distribution of all DNTTIP1 binding sites on promoters (TSS) and promoter distal elements in WT and Dnttipl KO1 mESCs sorted by enrichment in descending order in WT mESCs and compared to H3K4me1, H3K4me3, H3K27ac and H3K27me3 occupancy in WT and Dnttip1 KO1 mESCs. The color scale depicts the normalized ChIP-seq signal intensity ( $\log _{2} \mathrm{CPM}$ per $20 \mathrm{bp}$ bin).

B, C Occupancy plots displaying the average DNTTIP1 enrichment over gene bodies stratified by B transcriptionally down- $(\mathrm{FC}<-1.5, \mathrm{p}<0.01)$ or $\mathbf{C}$ upregulated $(\mathrm{FC}>1.5, \mathrm{p}<0.01)$ genes in WT versus Dnttipl KO1 mESCs.

D De novo motif analysis of DNTTIP1-bound genes that are down- or upregulated in Dnttip1 KO1 versus WT mESCs with motif-enrichment plot for ELK1 and RBFOX2.

E, F ChIP-seq profiles of the E Ntnl and F Idl loci for DNTTIP1 in WT, Dnttipl KO1 and Elmsan1 KO1 mESCs and for HDAC1 in WT mESCs. Promoter and putative enhancer regions used for manual ChIP experiments in Figure 4 and Figure S6 are highlighted by orange boxes.

Figure S6. MiDAC directly targets positive and negative regulators of neurite outgrowth during neural differentiation.

A-J qPCR from manual ChIP experiments against A, F DNTTIP1, B, G ELMSAN1, C, H HDAC1, D, I H3K27 ac and E, J H4K20ac from WT, Dnttipl KO1 and Elmsan1 KO1 NE targeting select promoter, putative enhancer and intragenic control regions of A-D the Ntn 1 or E-H the IdI loci as highlighted in Figure S5E and F. IgG was used as a control antibody. Unpaired t-test was performed throughout where ${ }^{* *}, \mathrm{p} \leq 0.01$; and $\mathrm{ns}, \mathrm{p}>0.05$ is not significant.

Figure S7. MiDAC does not transcriptionally regulate the receptors Robo3 and Unc5b of the SLIT3 and NTN1 signaling pathways.

A, B ChIP-seq profiles of the A Robo3 and B Unc5b loci for DNTTIP1 in WT, Dnttip1 KO1 and Elmsan1 KO1 mESCs and for HDAC1 in WT mESCs. Promoter regions used for manual ChIP experiments in Figure S7C and E are highlighted by orange boxes. 
C qPCR from manual ChIP experiments against DNTTIP1, ELMSAN1 and HDAC1 from WT, Dnttipl KO1 and Elmsan1 KO1 NE targeting the promoter of the Robo3 locus as highlighted in Figure S7A. IgG was used as a control antibody.

D qRT-PCR for Robo3 mRNA in WT, Dnttip1 KO1 and Elmsan1 KO1 mESCs. Expression was normalized to Gapdh.

E qPCR from manual ChIP experiments against DNTTIP1, ELMSAN1 and HDAC1 from WT, Dnttip1 KO1 and Elmsan1 KO1 NE targeting the promoter of the Unc5b locus as highlighted in Figure S7B. IgG was used as a control antibody.

F qRT-PCR for Unc5b mRNA in WT, Dnttipl KO1 and Elmsan1 KO1 mESCs. Expression was normalized to Gapdh.

Figure S8. MiDAC regulates neurite outgrowth via the SLIT3/ROBO3 and NTN1/UNC5B signaling pathways.

A Experimental outline of assay to rescue neurite outgrowth defects in Dnttipl KO1 or Elmsan1 $\mathrm{KO} 1 \mathrm{NE}$ as described in Figure 5A-C and 6B-D.

B Experimental outline of the chamber assay to rescue the neuronal network formation defects of granule neuron progenitor (GNP)-derived neurons co-cultured with Dnttip1 KO1 or Elmsan1 KO1 NE as described in Figure S8C, D or CM of Dnttip1 KO1 or Elmsan1 KO1 NE as described in Figure S8E, F.

C Chamber assay utilizing GNPs that were co-cultured in the lower chamber in parallel with differentiating WT, Dnttipl KO1 or Elmsan1 KO1 NE in the upper chamber comprising a time window from day 7-12 of differentiation to induce neuronal network formation from GNPs. Defined differentiation-inducing GNP medium was used as a positive and CM from mouse embryonic fibroblasts (MEFs) as a negative control. TUBB3 IF staining of GNP-derived neuronal networks was performed after 6 days of differentiation. Nuclei were stained with DAPI. The white scale bar represents $50 \mu \mathrm{m}$.

D Quantification of neuronal network formation from the TUBB3 IF staining in Figure S8C using ImageJ. The percentage of formed neuronal networks within the total population of TUBB3positive neurons is displayed. A neuronal network was scored when a closed local circuit was detected around an individual neuron. Neuronal network formation was assessed for 100 neurons per sample in triplicate. 
E Chamber assay to rescue the network formation defects of GNP-derived neurons that were supplemented with CM of Dnttipl KO1 NE. CM of Dnttipl KO1 NE was supplemented with the recombinant signaling ligands SLIT3 and/or NTN1 from day 7-12 without or with pre-blocking of GNP-derived neurons with control IgG or signaling receptor antibodies against ROBO3 and/or UNC5B. TUBB3 IF staining was performed after 12 days of differentiation. Nuclei were stained with DAPI. The white scale bar represents $50 \mu \mathrm{m}$.

F Quantification of neuronal network formation from the TUBB3 IF staining in Figure S8E using ImageJ. The percentage of formed neuronal networks within the total population of TUBB3positive neurons is displayed. A neuronal network was scored when a closed local circuit was detected around an individual neuron. Neuronal network formation was assessed for 100 neurons per sample in triplicate.

Unpaired t-test was performed throughout where ***, $\mathrm{p} \leq 0.01$. 


\section{Titles and Legends for Supplemental Tables}

Table S1. List of guide RNA and deep sequencing primer sequences used for CRISPR/Cas9mediated genome editing of Dnttipl and Elmsan1 in mESCs.

Table S2. Differentially expressed genes in Dnttip1 KO (KO1 and KO2) and Elmsan1 KO (KO1 and $\mathrm{KO} 2)$ mESCs $(\mathrm{FC} \leq-1.5$ or $\geq 1.5$; p-value $<0.01)$ (MS Excel spreadsheets).

Table S3. DNTTIP1 binding sites identified by ChIP-seq in mESCs (MS Excel spreadsheet).

Table S4. Genes that are up- or downregulated in Dnttip1 KO (KO1 and KO2) versus WT mESCs

(FC $\leq-1.5$ or $\geq 1.5$; p-value $<0.01$ except for Spry4) and bound by DNTTIP1 (MS Excel spreadsheet).

Table S5. List of primer sequences used for qRT-PCR analysis.

Table S6. List of primer sequences used for ChIP qPCR analysis. 
A

MiDAC Subunits

DNTTIP1, 329 aa $\square \square=\square$

ELMSAN1, 1045 aa $\square$

TRERF1, 1200 aa

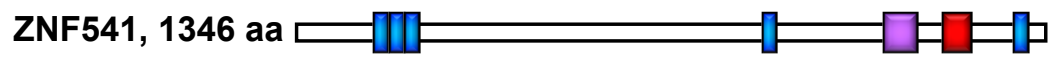

HDAC1, 482 aa

HDAC2, 488 aa r

Zinc finger DELM2 ISANT DDimerization [Ski/Sno/Dac DHDAC
D

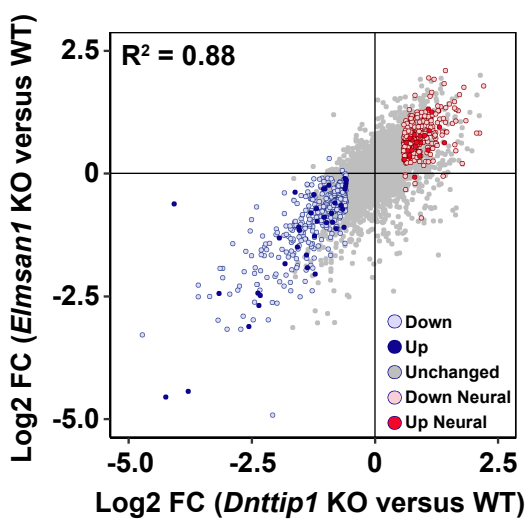

E MiDAC-regulated genes in mESCs

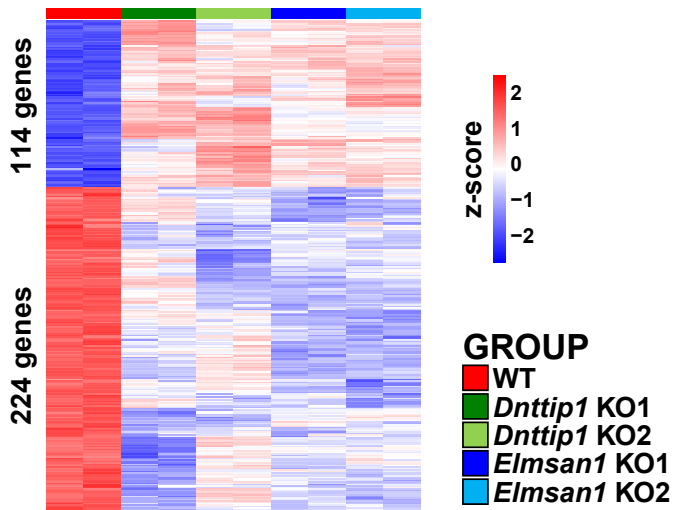

Elmsan1 KO2
$\mathbf{F}$

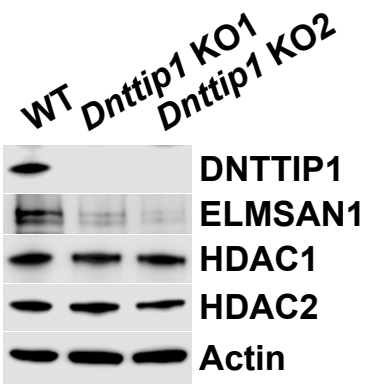

C

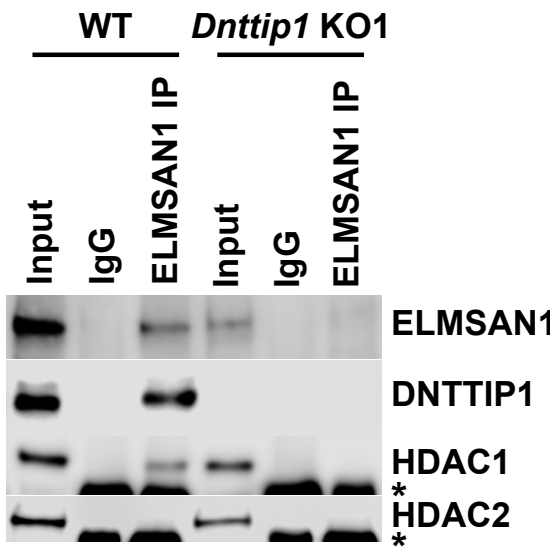

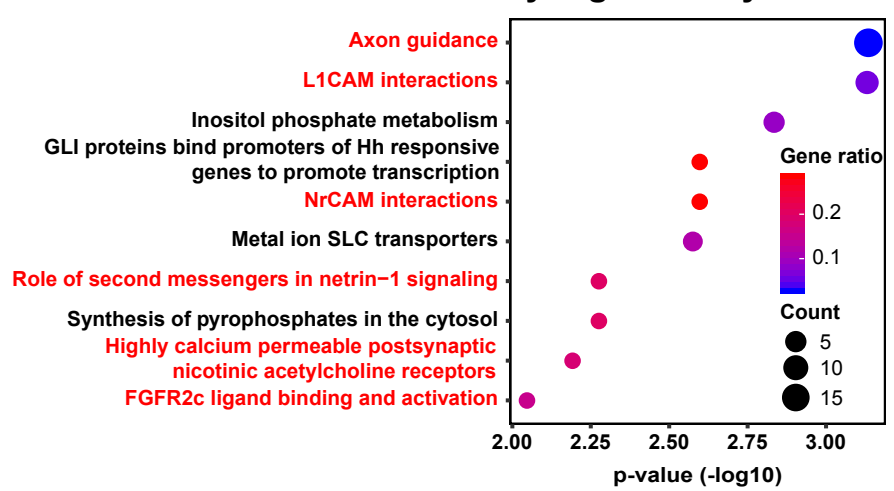

G Neurodevelopmental genes controlled by MiDAC
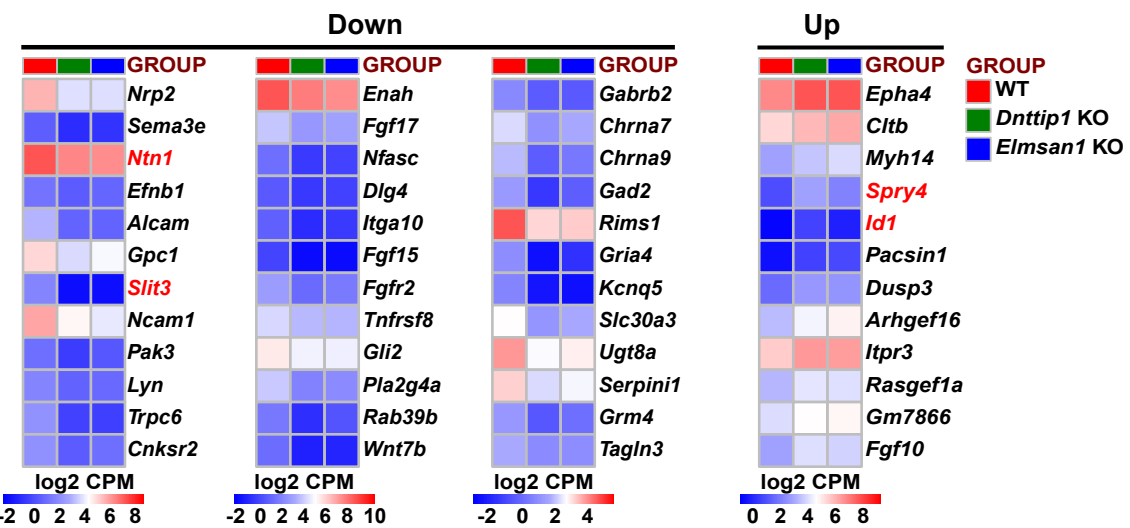
A

C

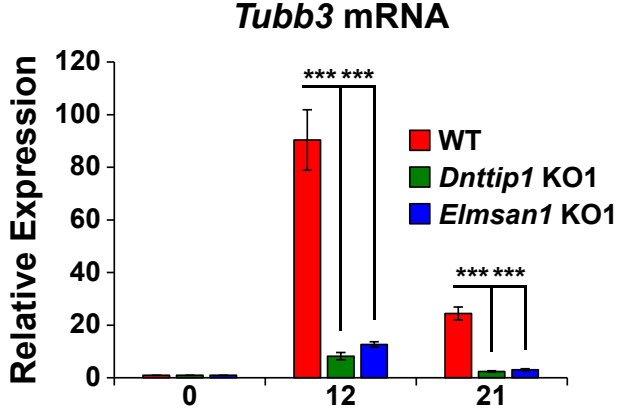

Time of NE differentiation (in days)

D

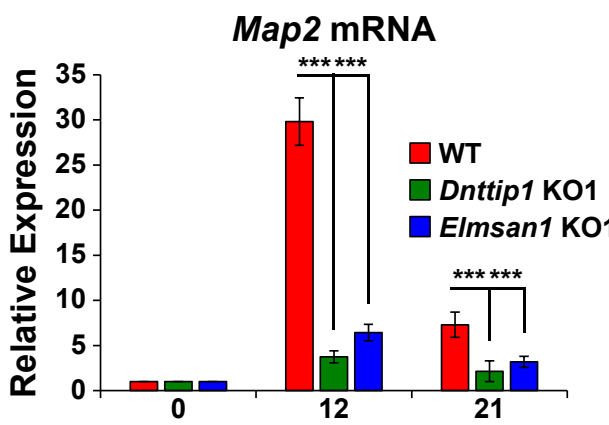

Time of NE differentiation (in days)

E

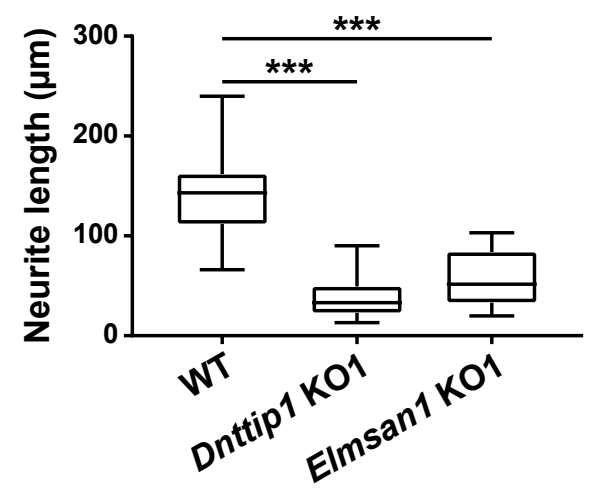

B



mESCs in differentiation medium

+ Retinoic acid (RA)

Day 7-12

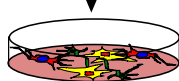

Day $8 \swarrow$ Day 12

PAX6 TUBB3, MAP2

Tubb3 mRNA

Day 1 mescs in differentiation medium

EBs seeded onto a gelatin-coated plate

Figure 2 


\section{Figure 3}

A
Genome-wide DNTTIP1
peak distribution

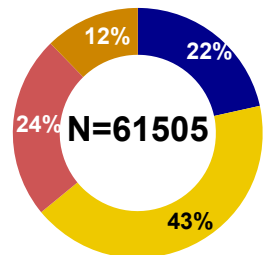

TSS

Intragenic

Upstream

Downstream
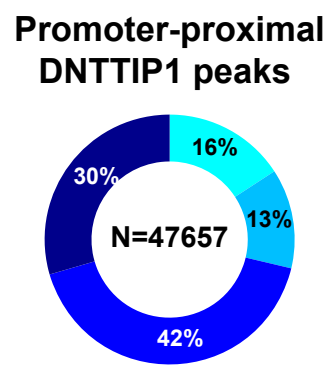

1-5 kb

5-10 kb

$10-50 \mathrm{~kb}$

$>50 \mathrm{~kb}$
B

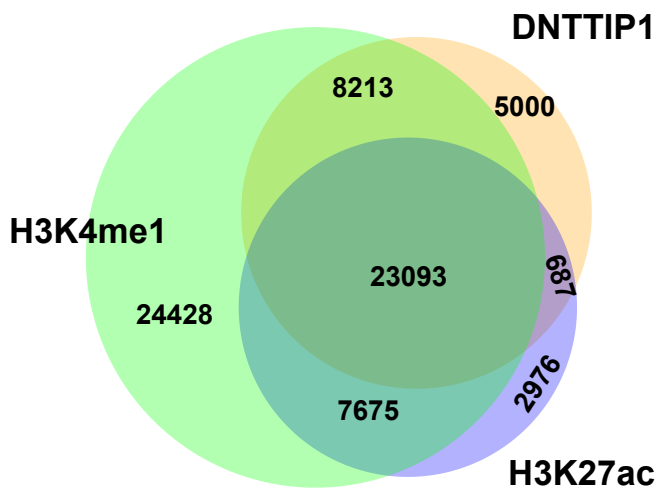

C

E
H

DNTTIP1

22036

30187

135358

Down genes

(493)

$p=8.39 \times 10^{-12}$

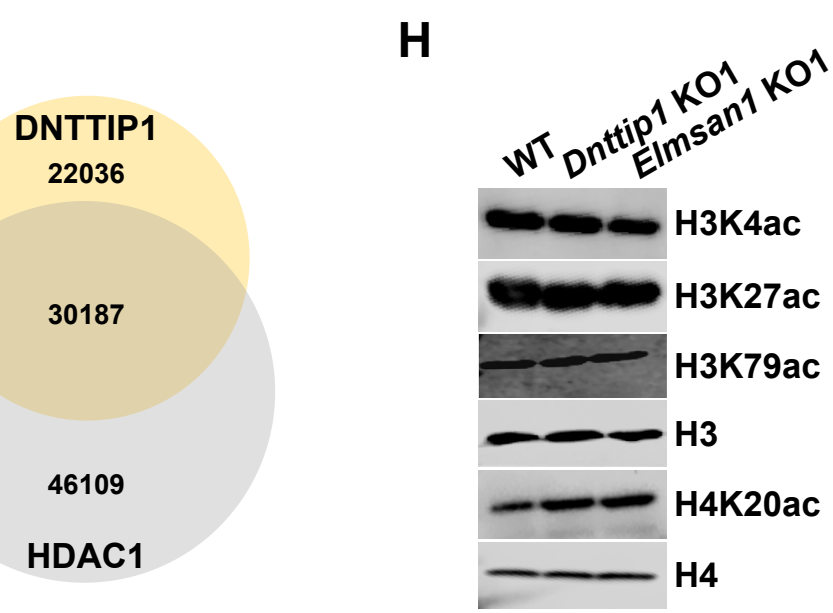

$314 \quad 54$

Up genes

(368)

$p=4.74 \times 10^{-11}$
F
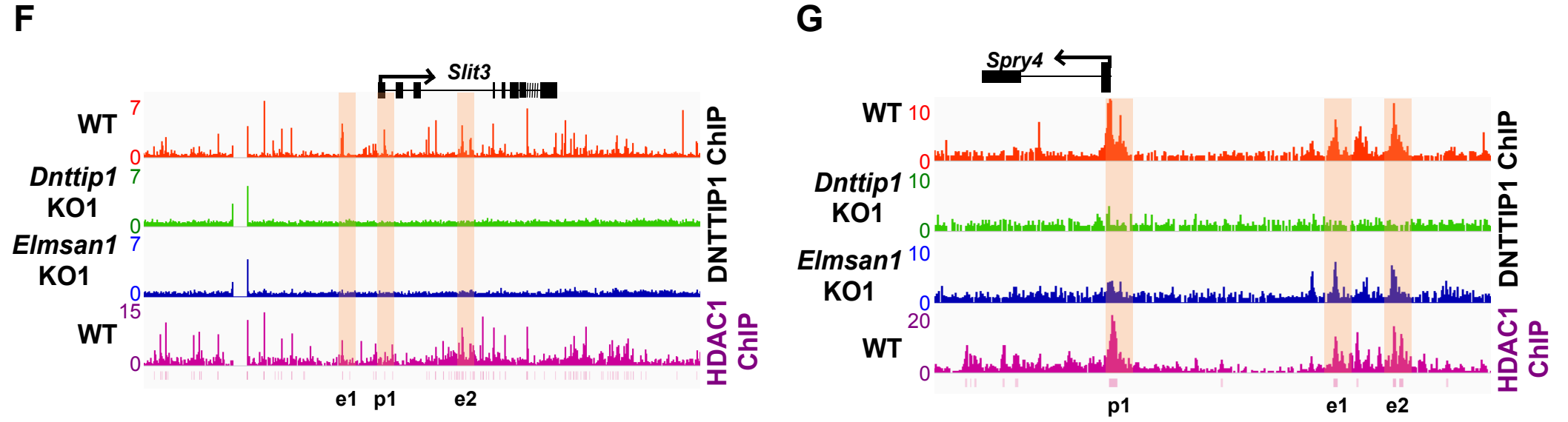
bioRxiv preprint doi: https://doi.org/10.1101/2020.04.07.02975- this version posted April 7, 2020. The copyright holder for this preprint (which was not certified by peer review) is the author/funder, who ra available under aCC 4.0 international license.

\section{Regulatory regions of the Slit3 locus}

A

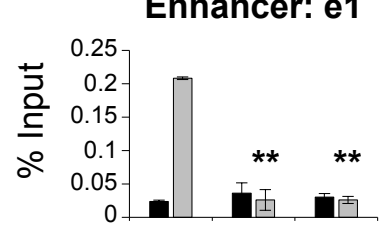

B

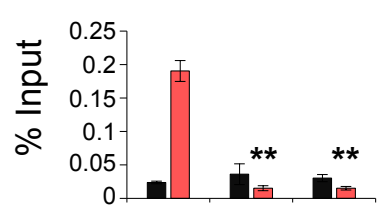

C

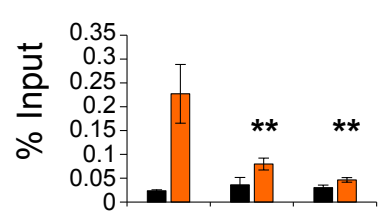

D

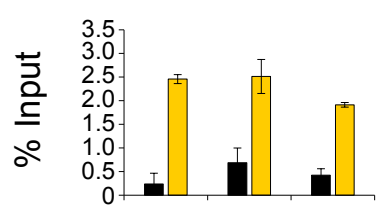

E

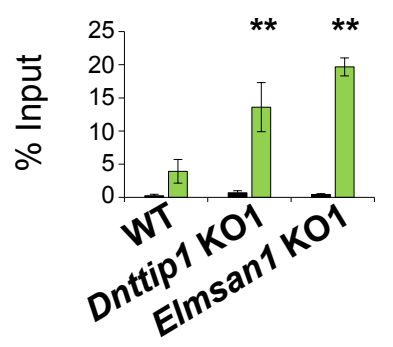

Promoter: p1
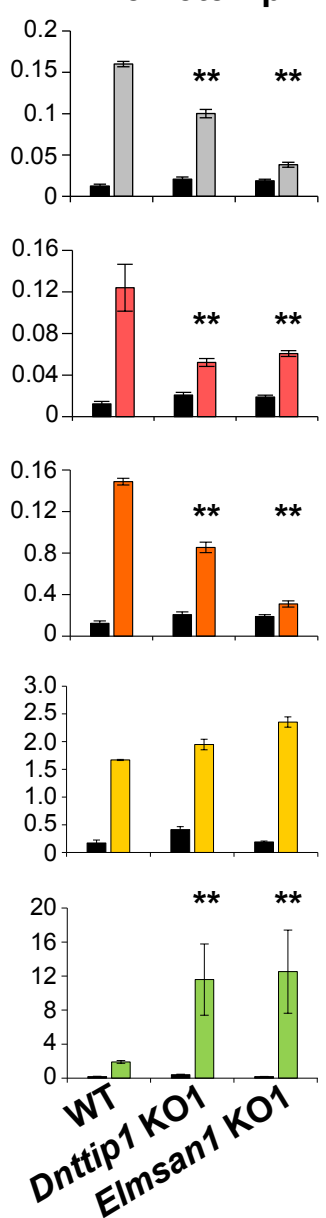

Enhancer: e2
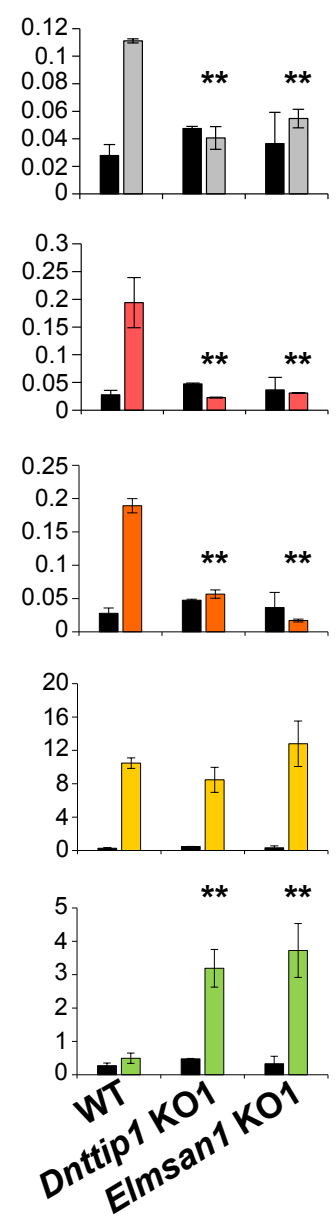

Intragenic control region
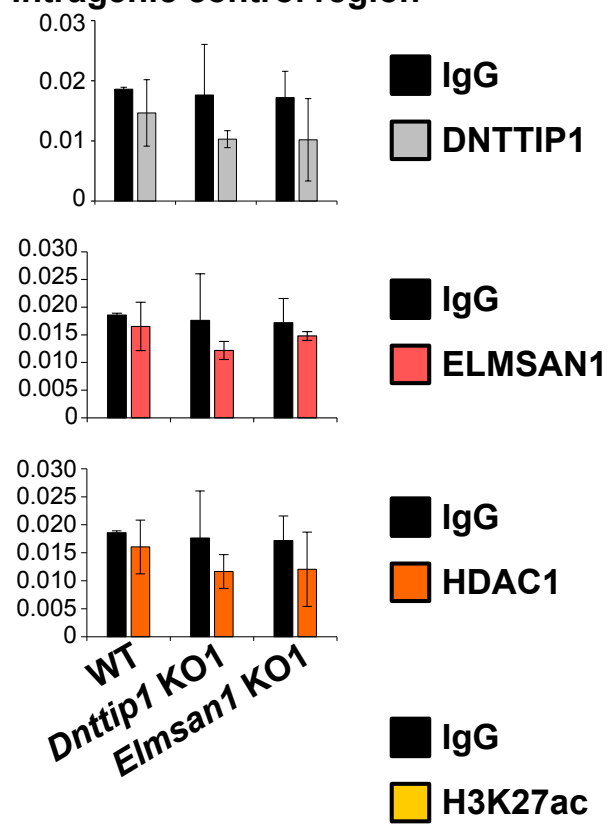

\section{Regulatory regions of the Spry4 locus}

$\mathbf{F}$

G
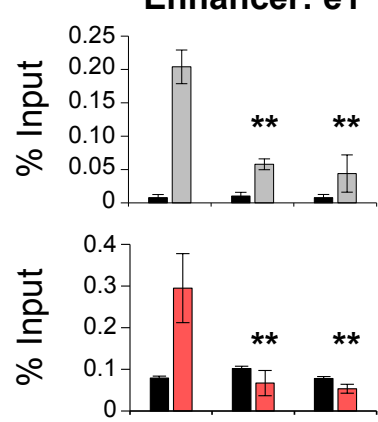

H

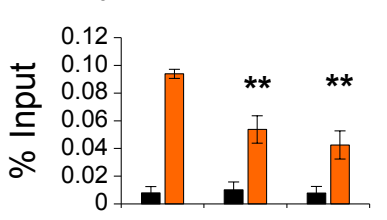

I

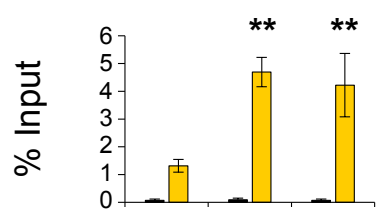

J

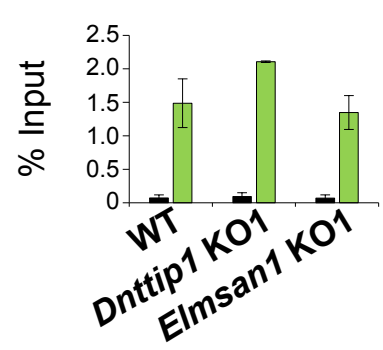

Promoter: p1
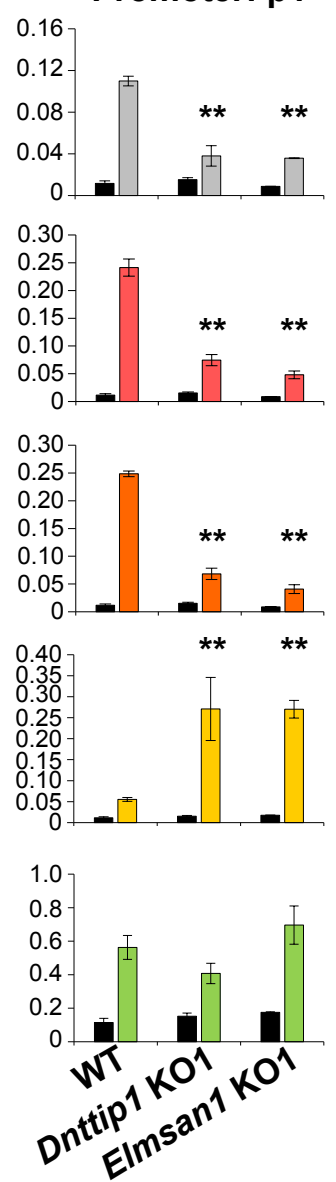

Enhancer: e2
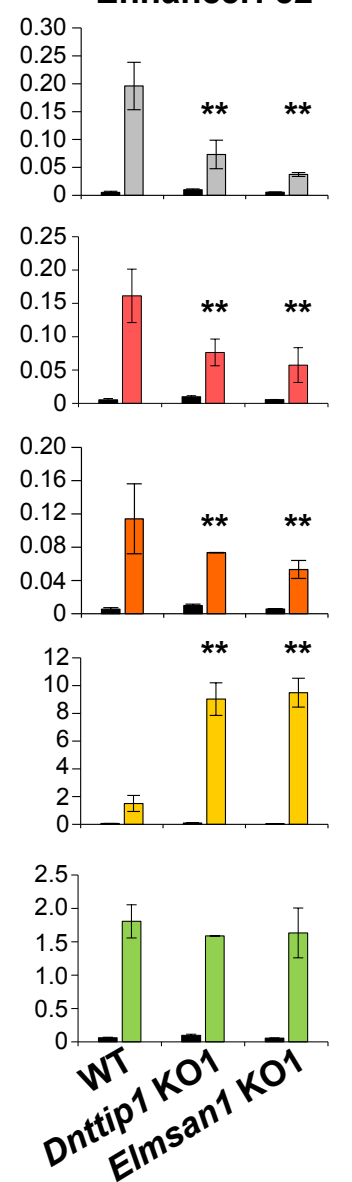

Intragenic control region
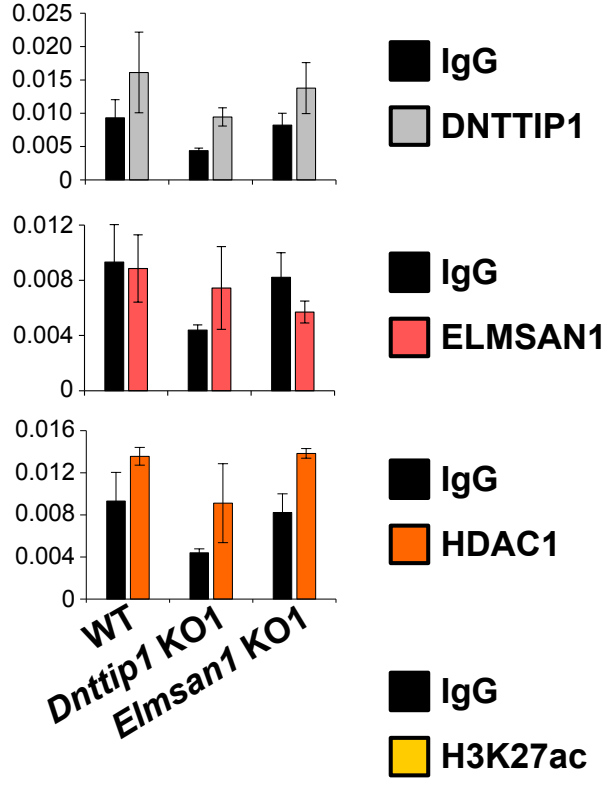

$\lg G$ 

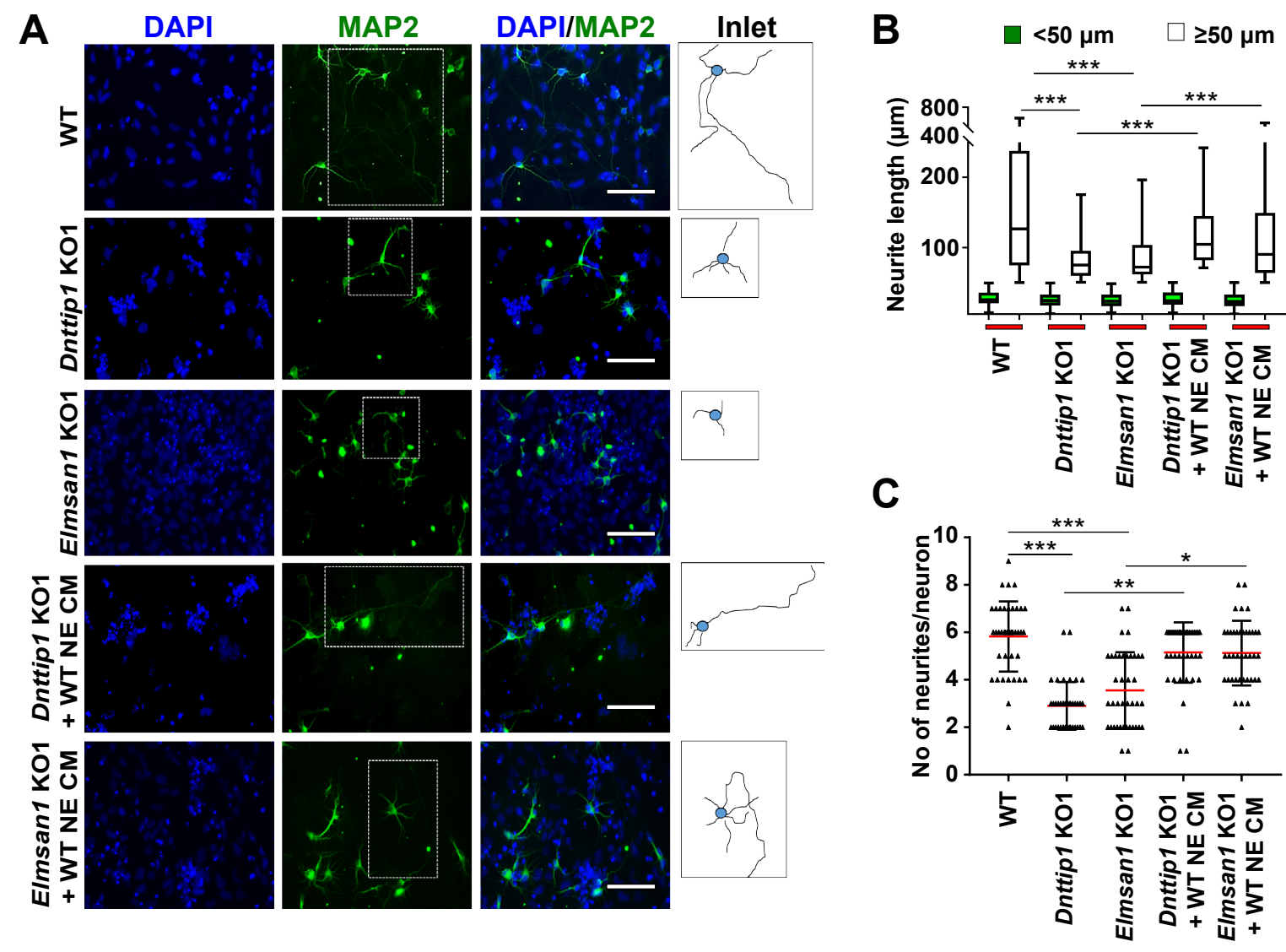


\section{Figure 6}

A

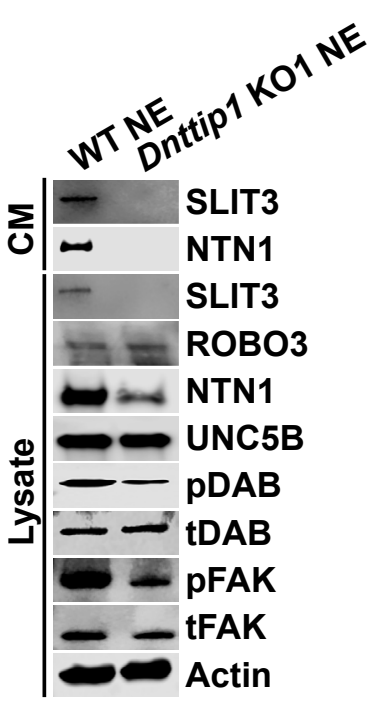

C

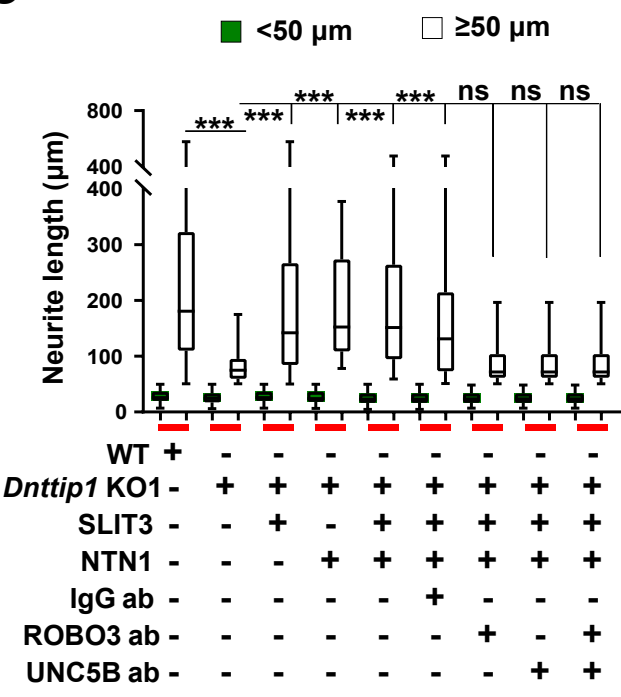

D

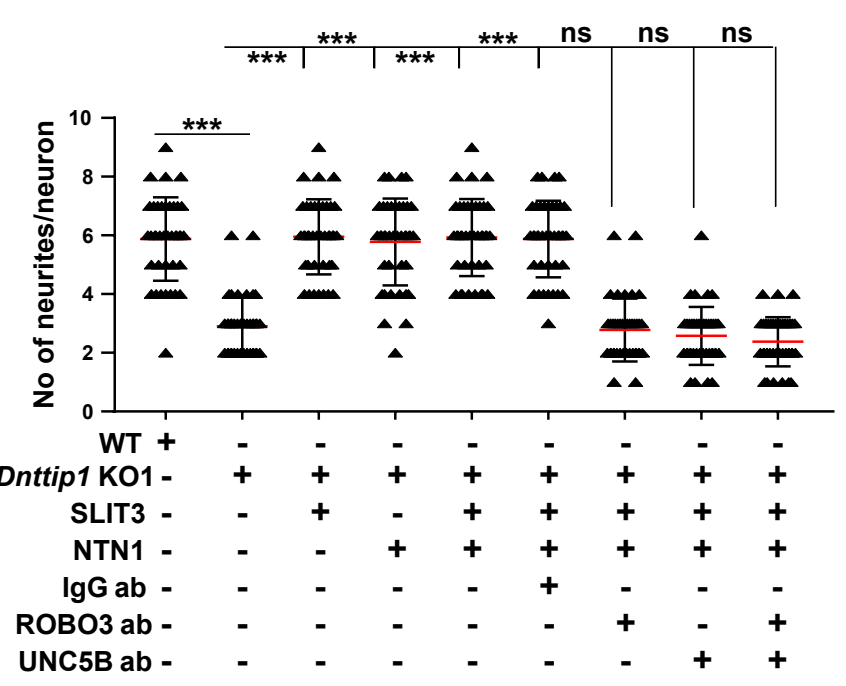

B




bioRxiv preprint doi: https://doi.org/10.1101/2020.04.07.029751; this version posted April 7, 2020. The copyright holder for this preprint (which was not certified by peer review) is the author/funder, who has granted bioRxiv a license to display the preprint in perpetuity. It is made
available under aCC-BY 4.0 International license.

\section{Ffigure 7}
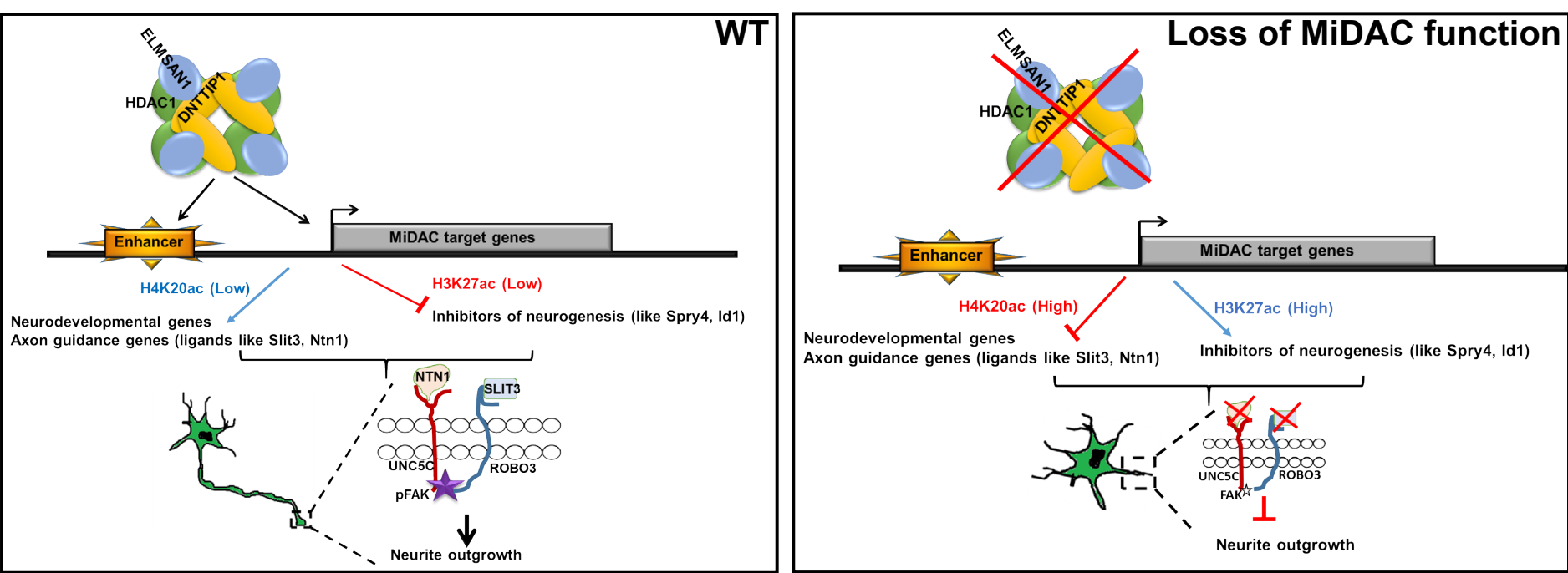
Figure S1

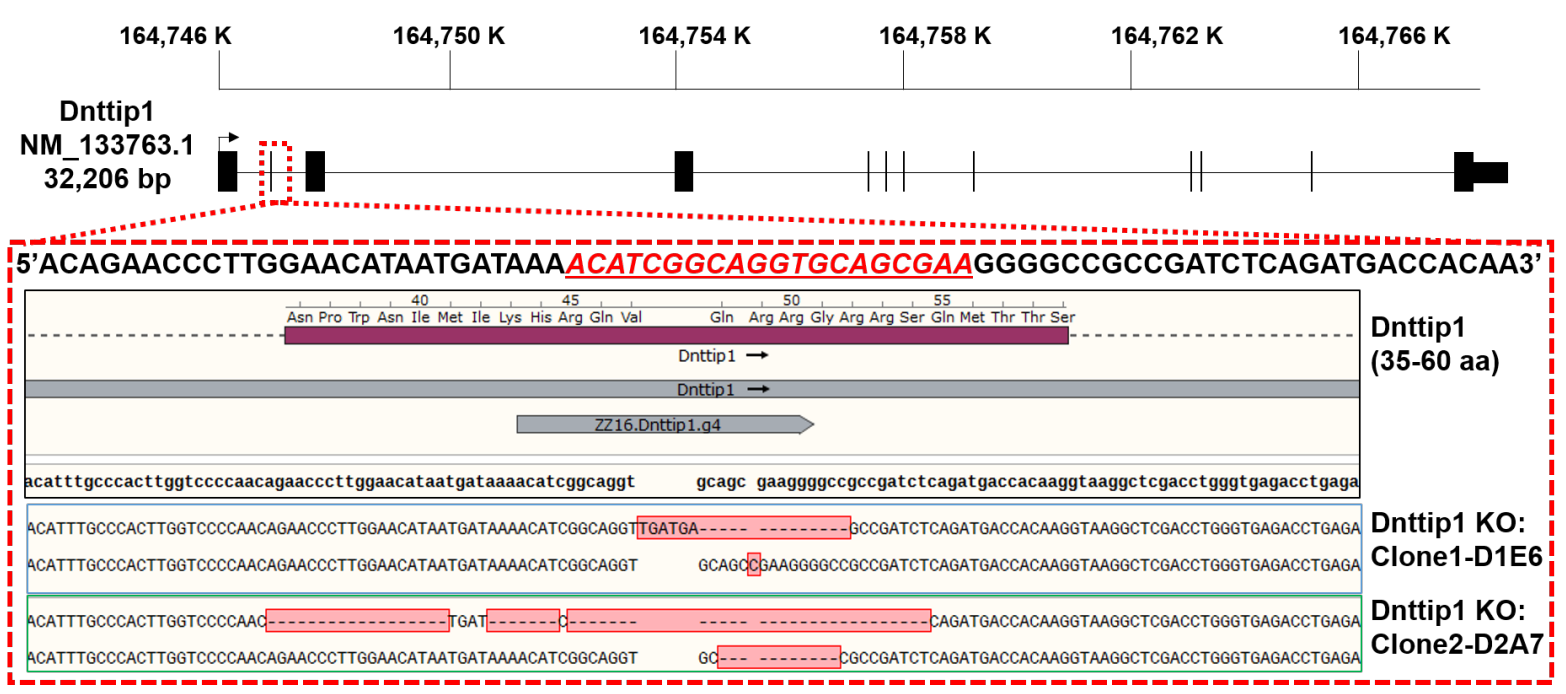

Elmsan1
NM_001043318
$\begin{gathered}7721 \mathrm{bp} \\ \text { 85, }\end{gathered}$

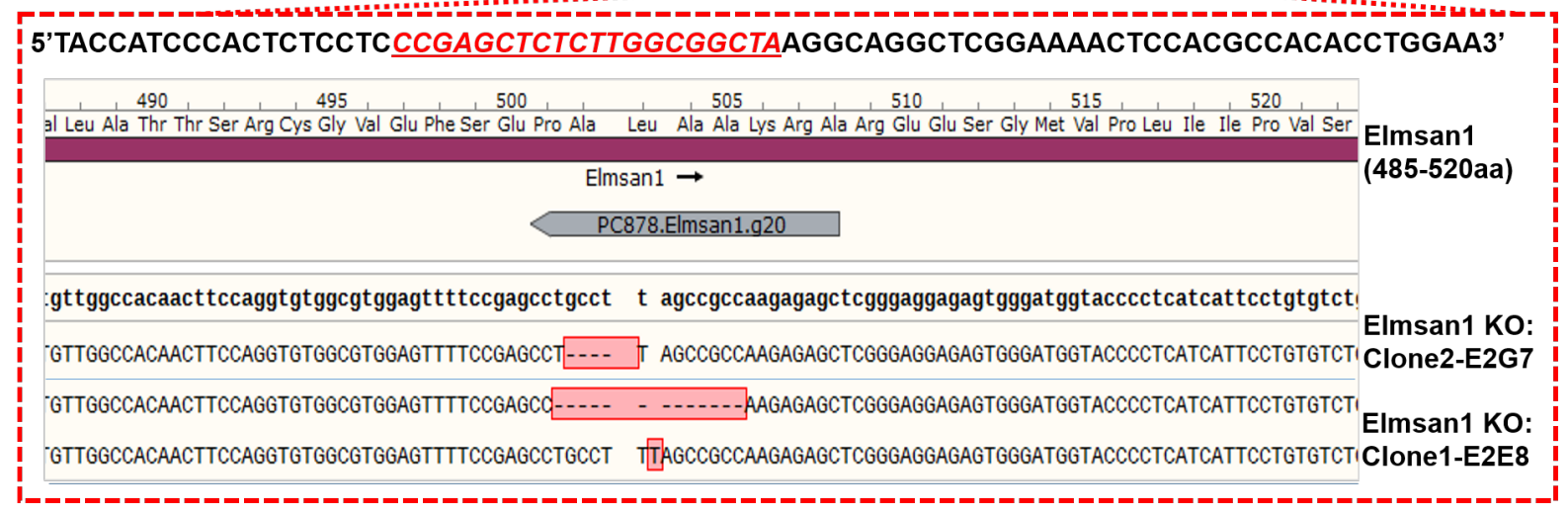

C

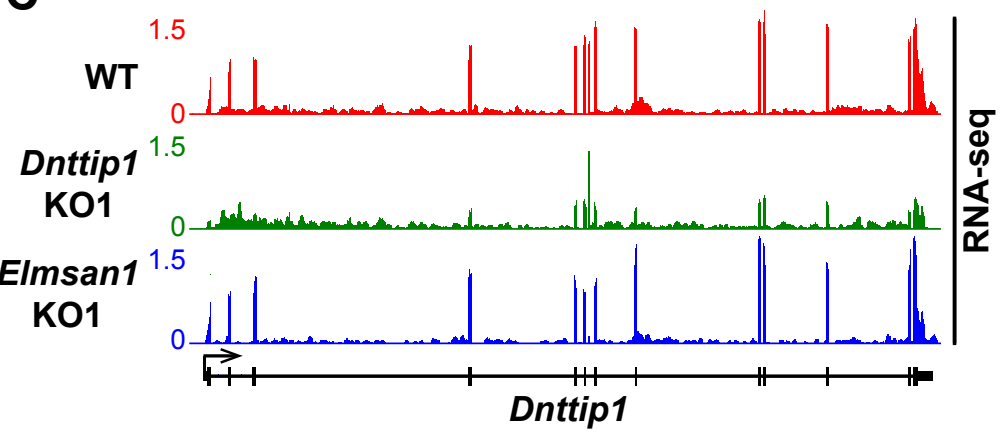

E

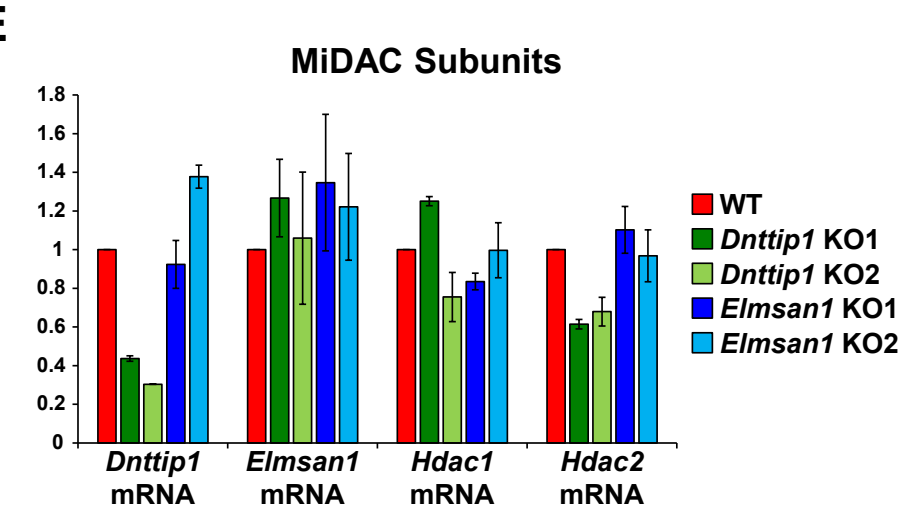

F
D
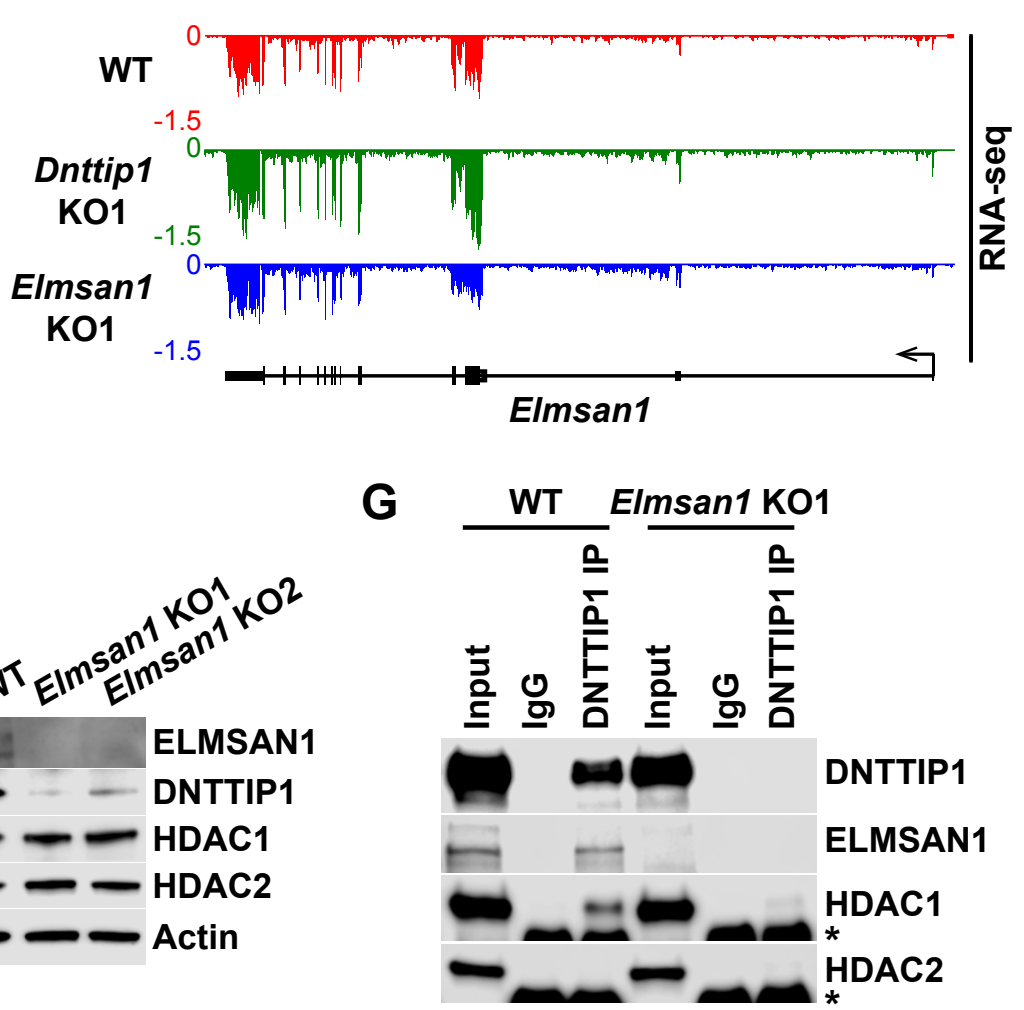


\section{Figure S2}

A

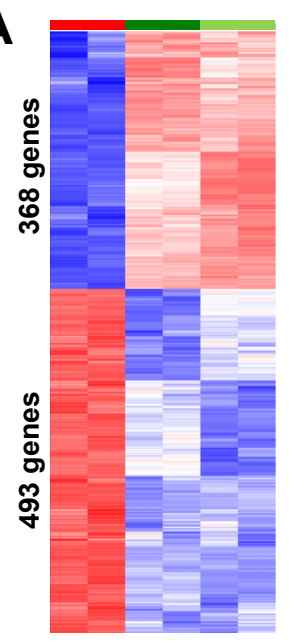

B

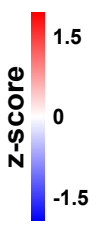

GROUP

WT

Dnttip1 K01

Dnttip1 KO2

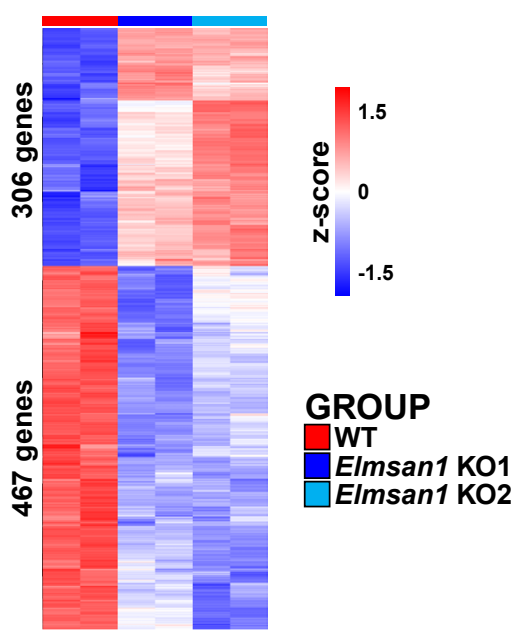

C

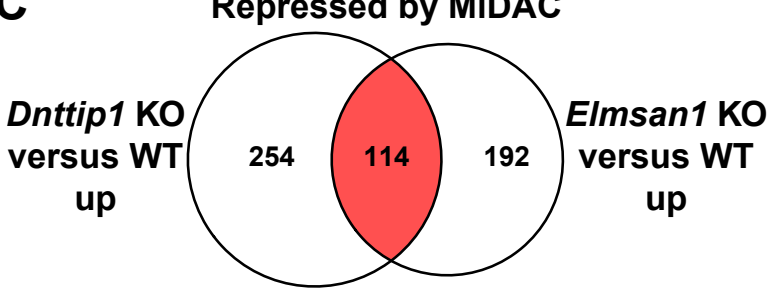

Activated by MiDAC

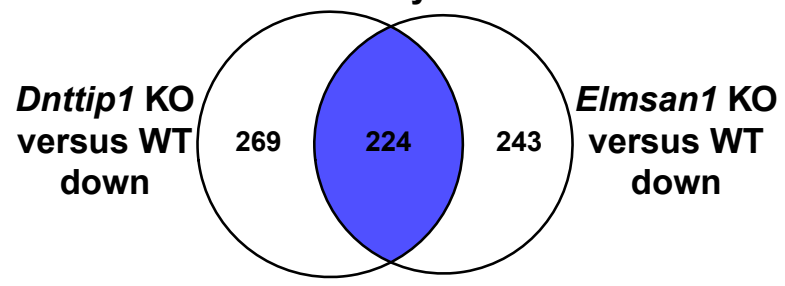

D

Downregulated in Dnttip1 KO versus WT

Transmission across chemical synapses

Metal ions SLC transporters

Neurotransmitter receptor binding and downstream transmission in post-synaptic cells

NrCAM interaction

Glutamate binding, activation of AMPA receptors and synaptic plasticity

Trafficking of AMPA receptor

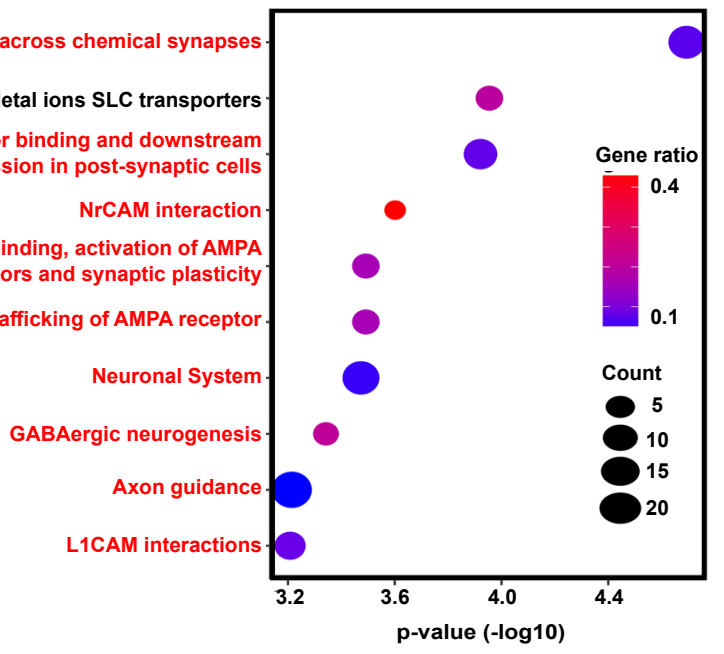

$E$

$\mathbf{F}$

\section{Downregulated genes in Elmsan1 KO versus WT}

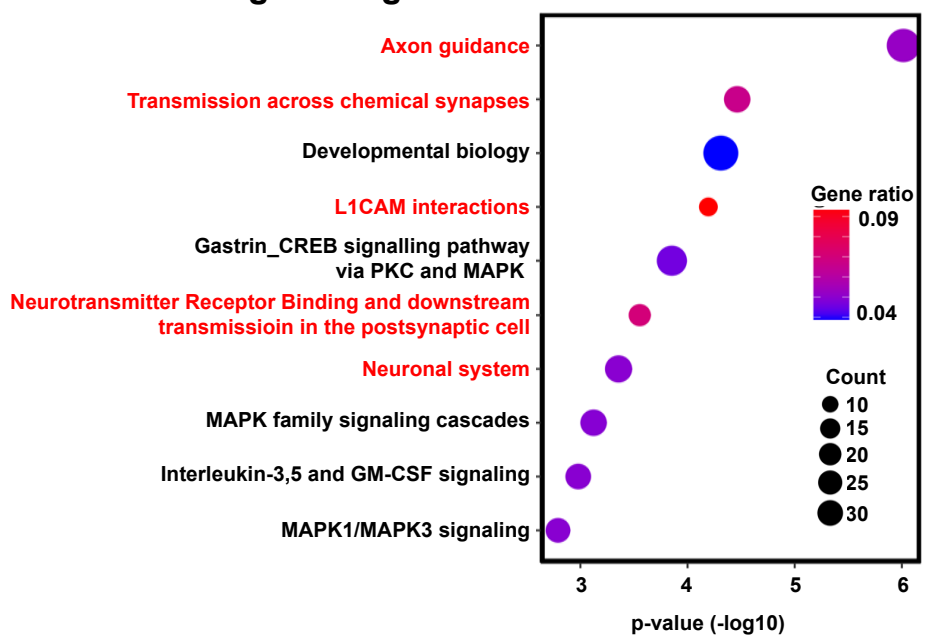

G

Upregulated genes in Elmsan1 KO versus WT

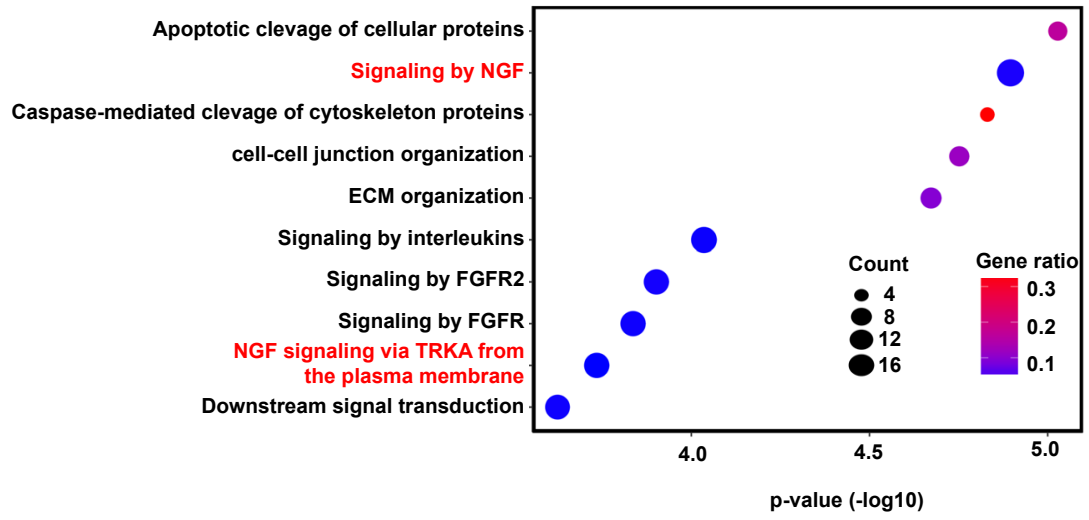


A
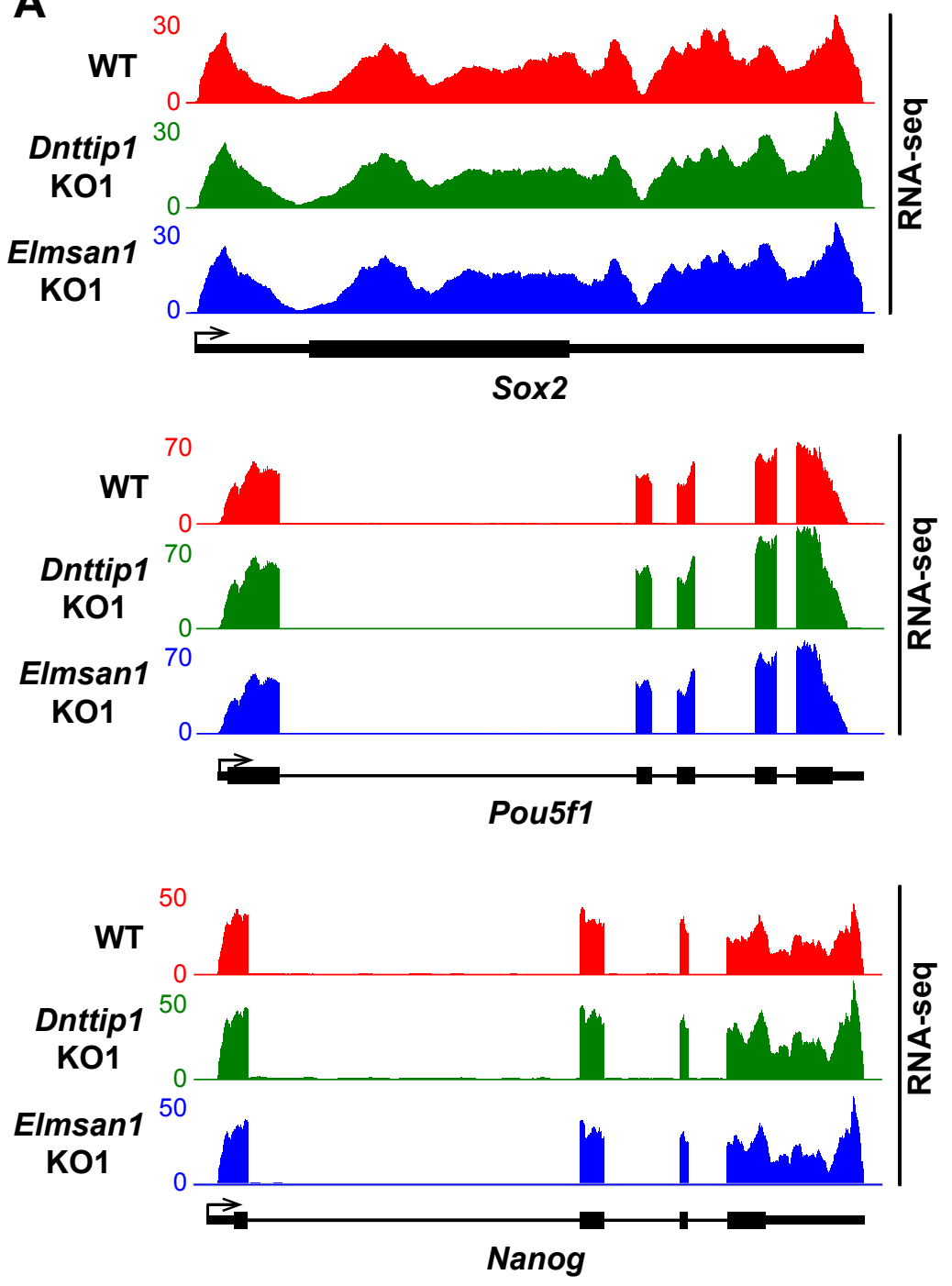

C

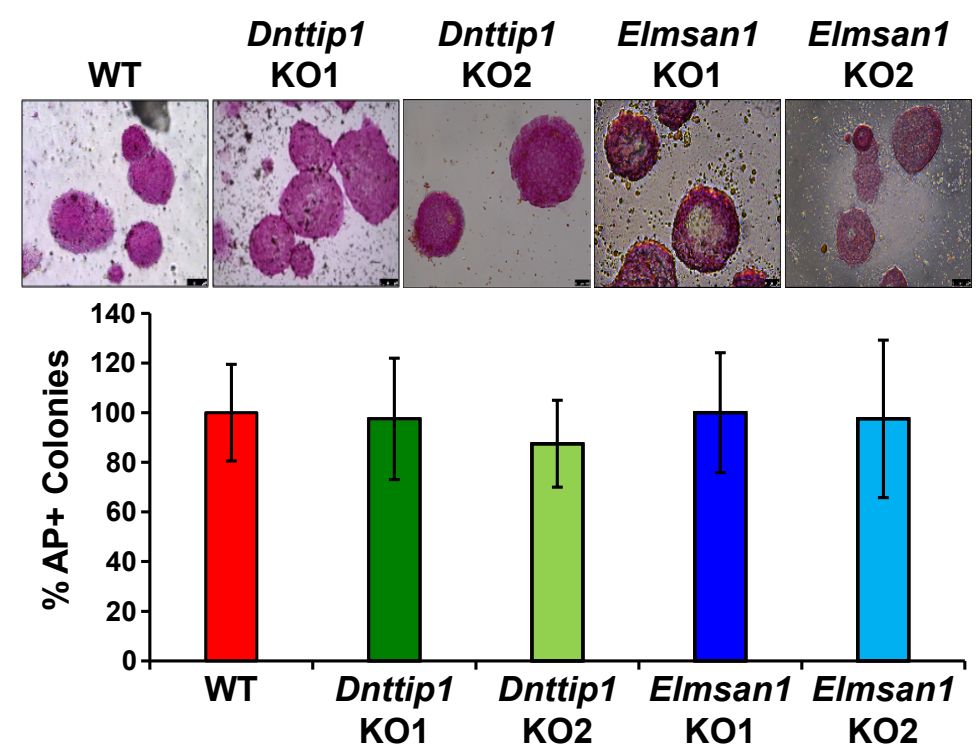

B
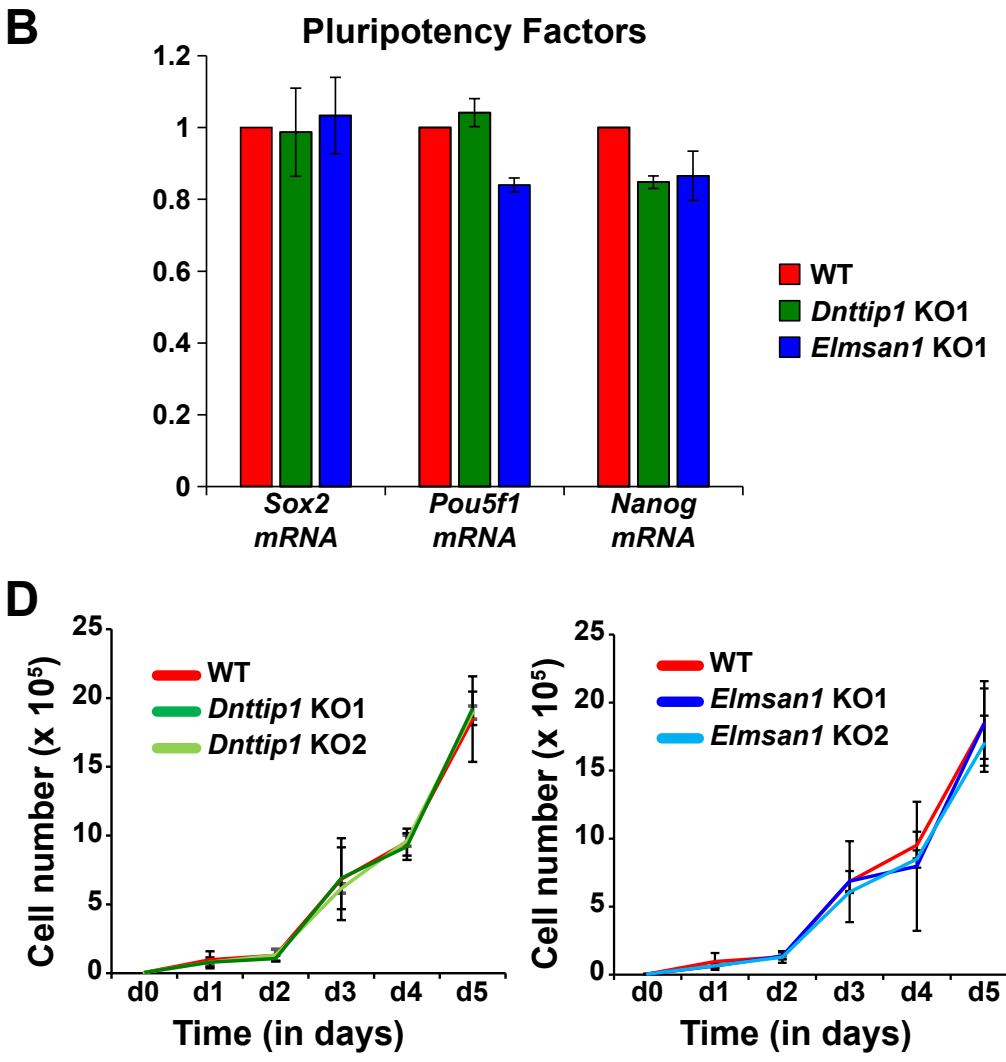

E
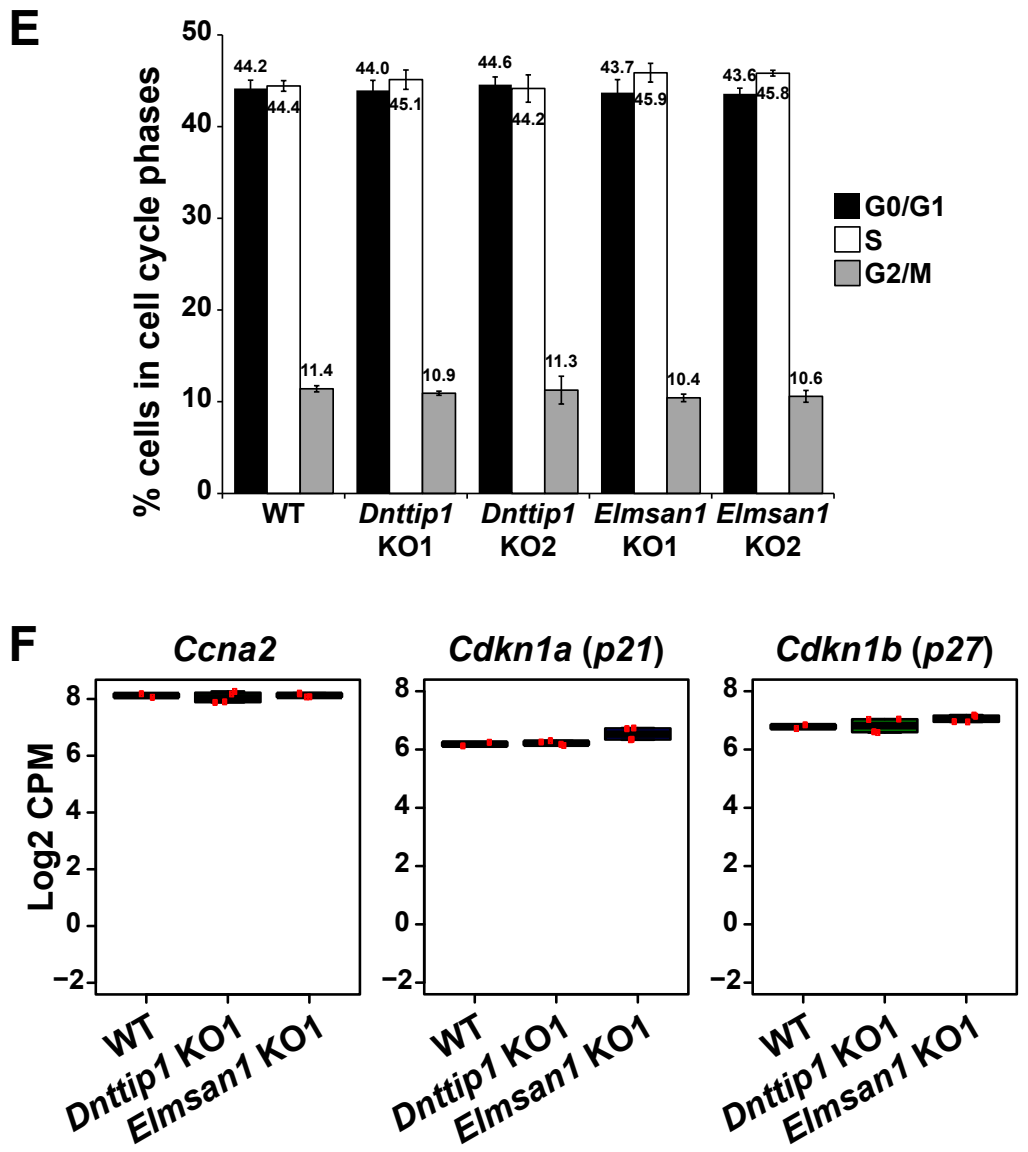


\section{Figure S4}

A

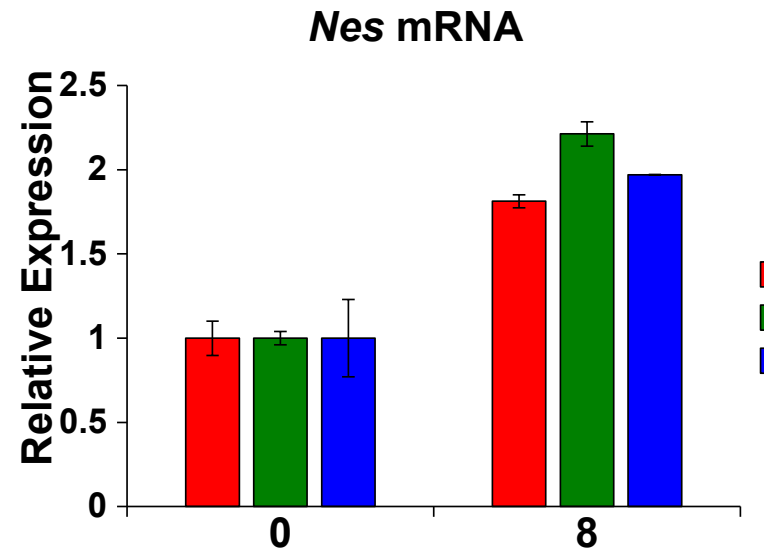

Time of NE differentiation (in days)
B

WT

Dnttip1 K01

Elmsan1 KO1
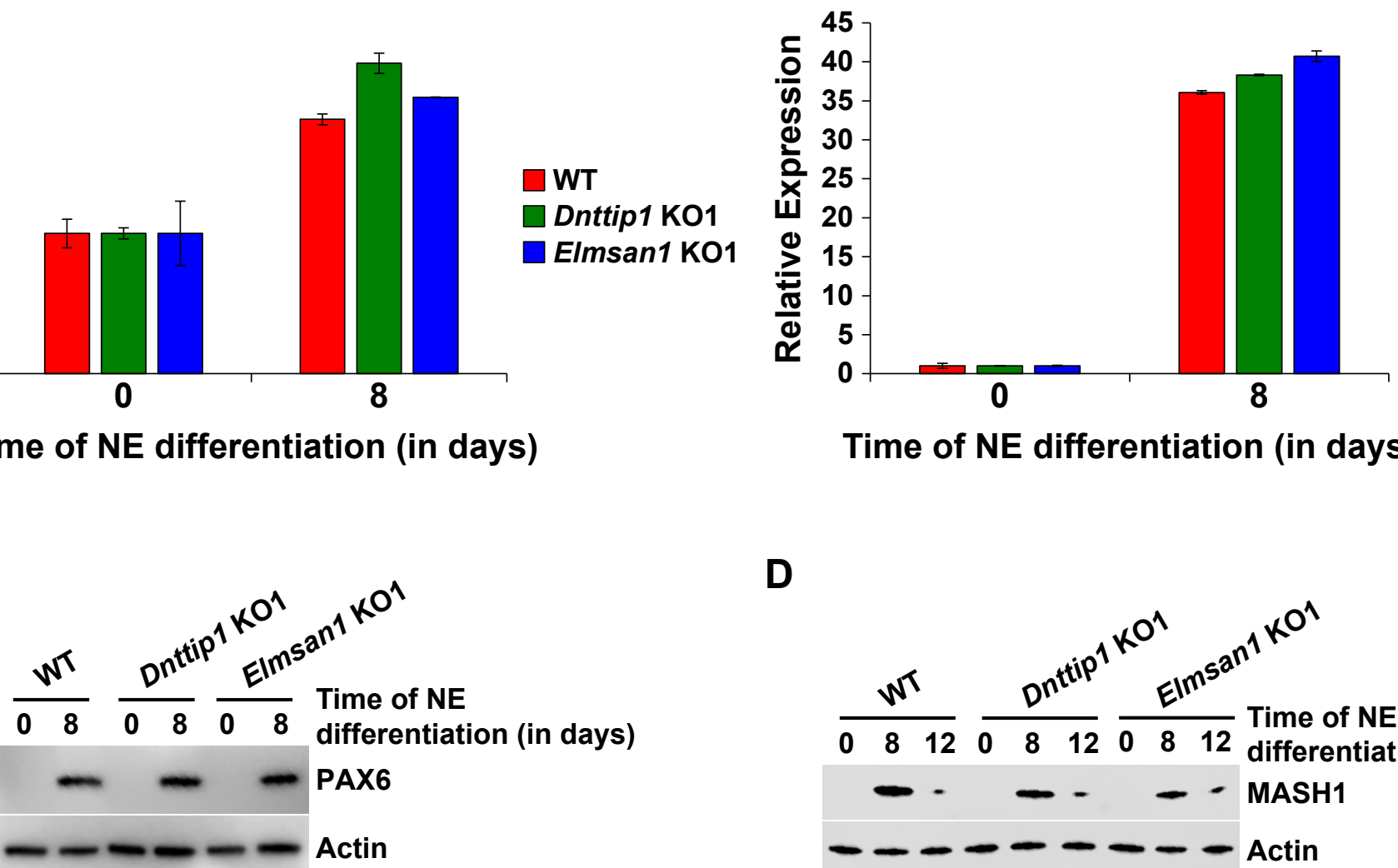

Time of NE differentiation (in days)

C

E

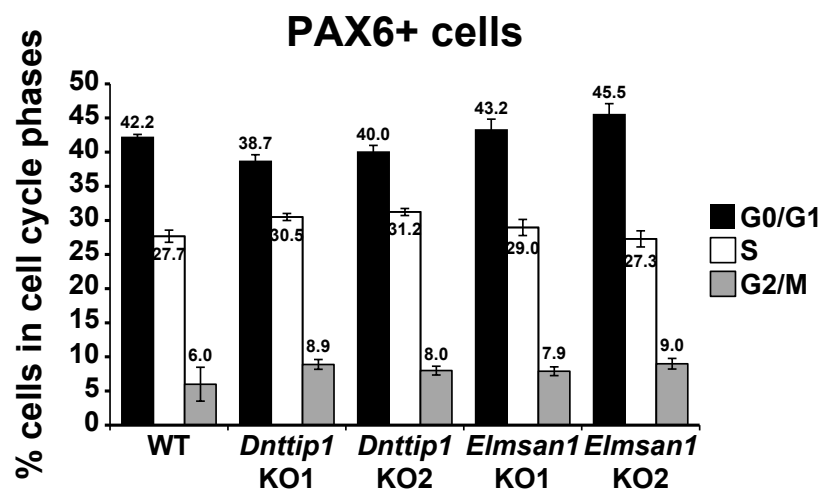

Pax6 mRNA

WT

Dnttip1 K01

IImsan1 KO1
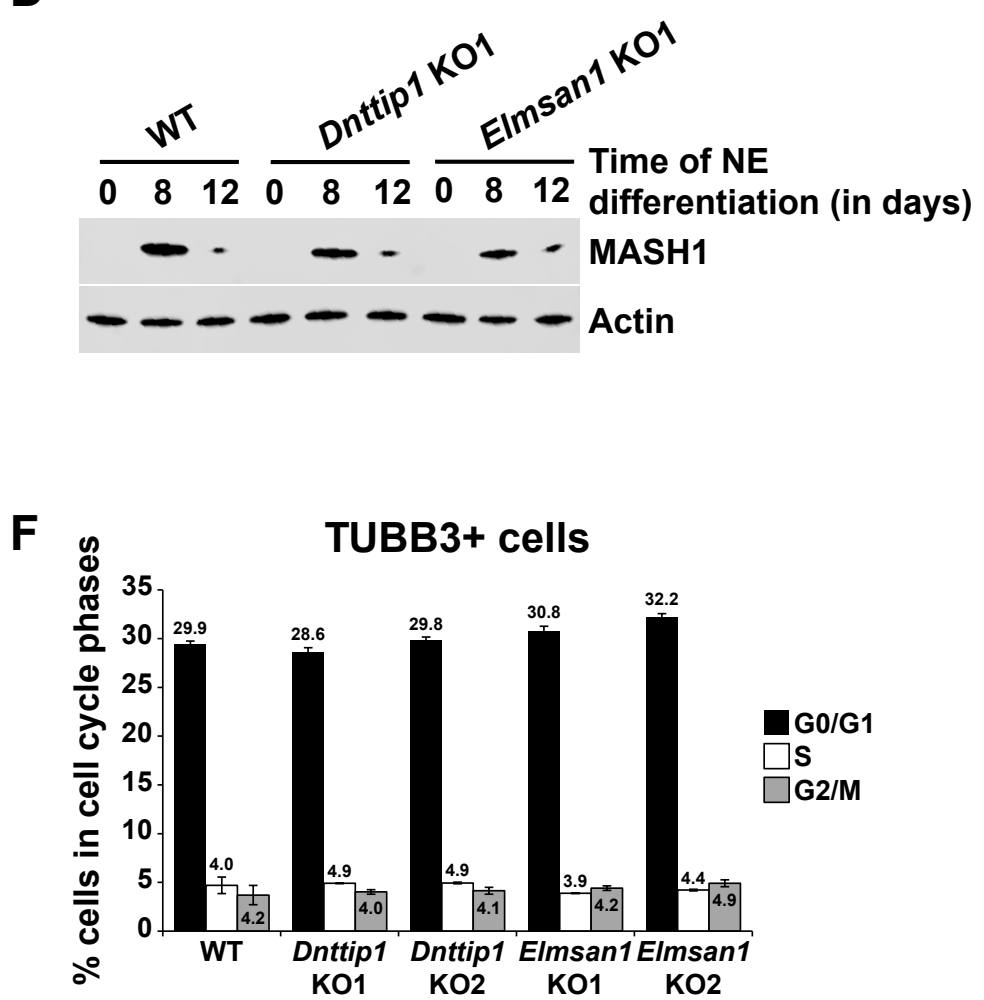


\section{Figure S5}

A

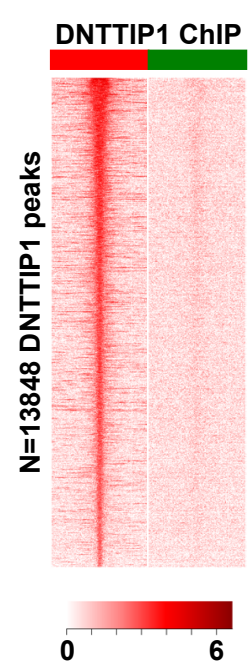

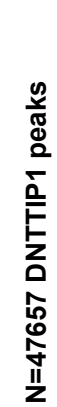

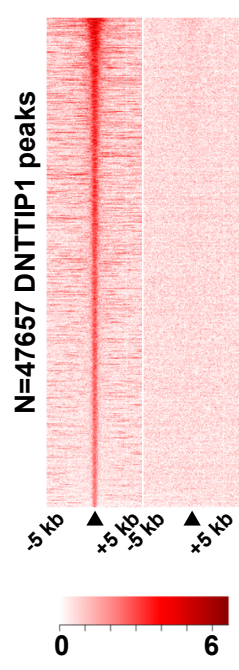

Promoter

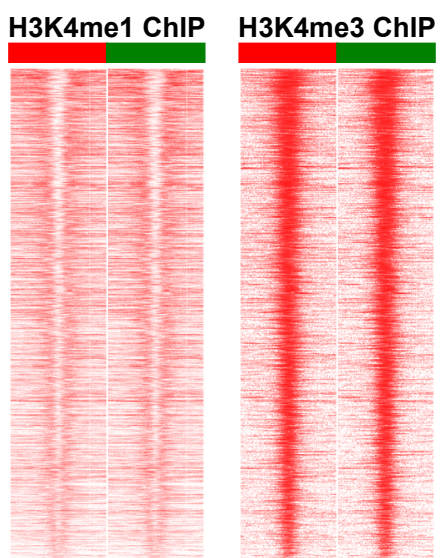

Normalized ChIP-seq signal intensity $(\log 2)$
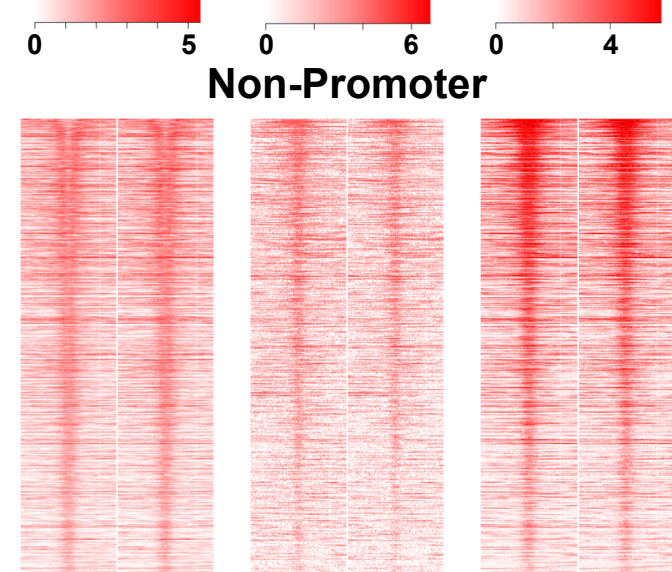

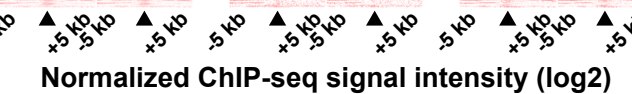

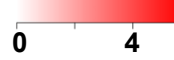

0

$0 \quad 6$
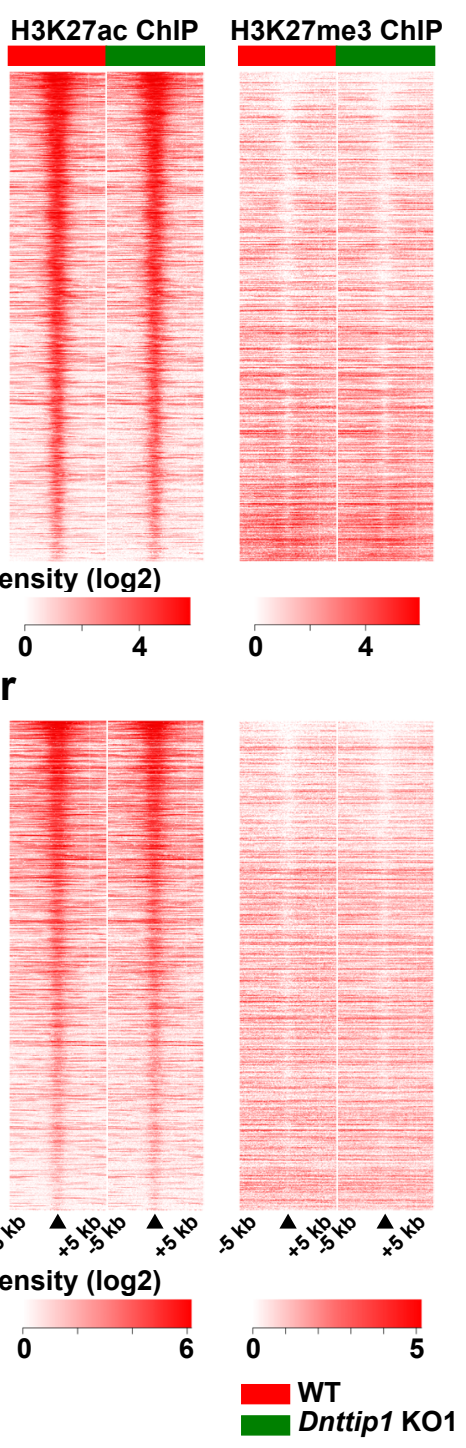

B

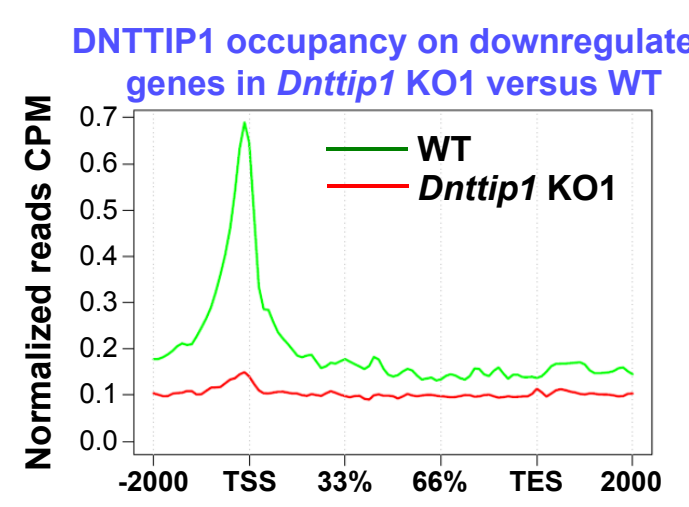

C
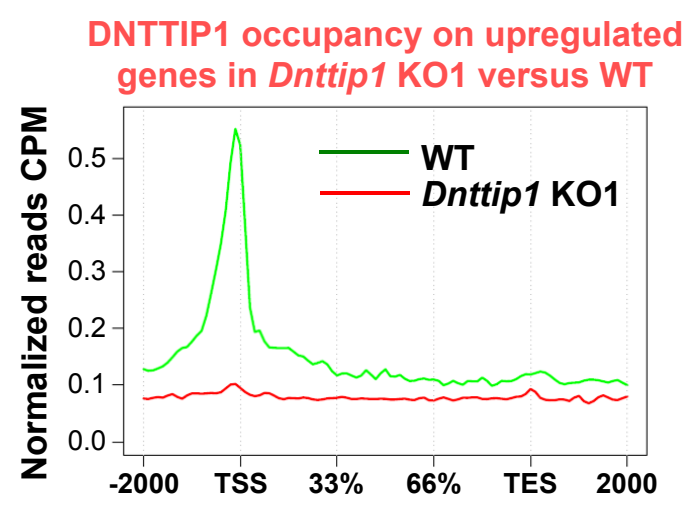

D

\begin{tabular}{|c|c|c|c|c|}
\hline Factor & $\begin{array}{c}\text { DEG } \\
\text { Category }\end{array}$ & Motif & $\begin{array}{l}\% \text { Target } \\
\% \text { Background }\end{array}$ & p-value \\
\hline ELK1 & Down & 숭술들 & $\begin{array}{l}19.15 \\
11.33\end{array}$ & $10^{-187}$ \\
\hline RBFOX2 & Up & ATCO & $\begin{array}{l}37.14 \\
29.55\end{array}$ & $10^{-12}$ \\
\hline
\end{tabular}

E

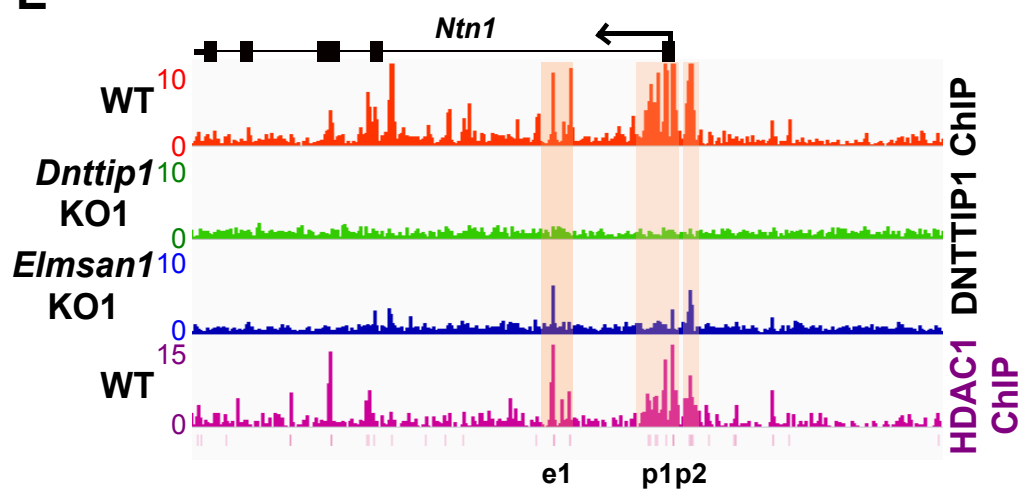

F
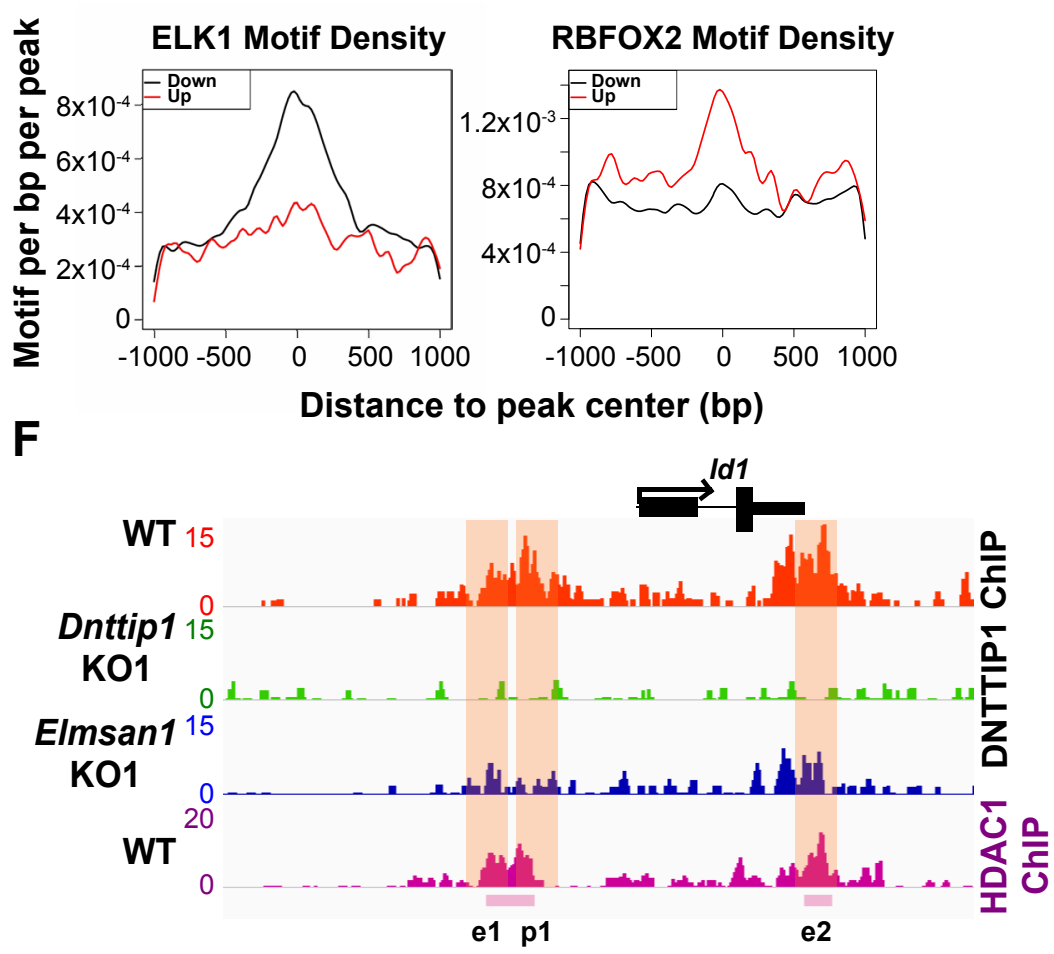
Figure S6

\section{Regulatory regions of the Ntn1 locus}

A

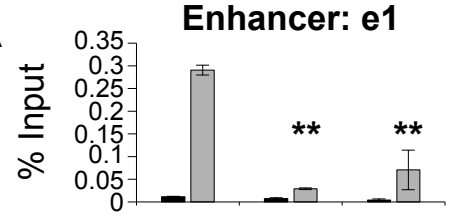

B

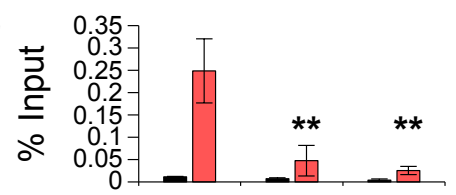

C

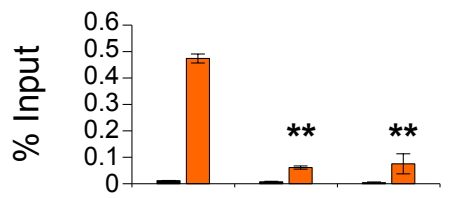

D

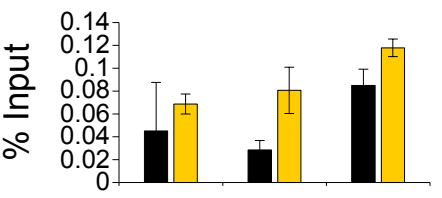

E

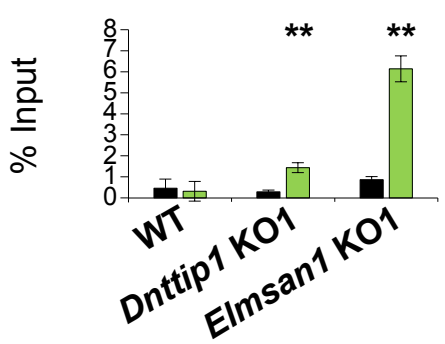

$\mathbf{F}$

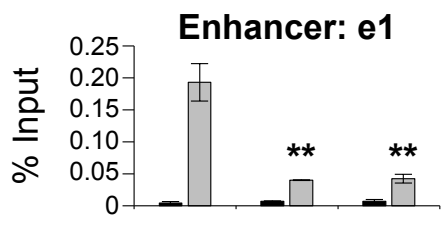

G

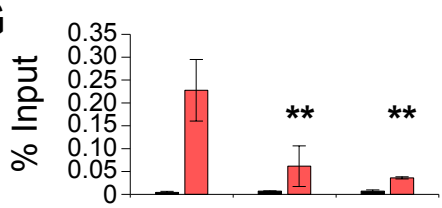

H

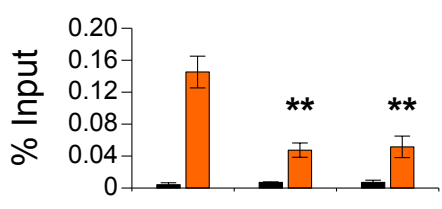

I

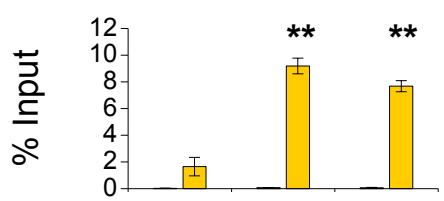

J

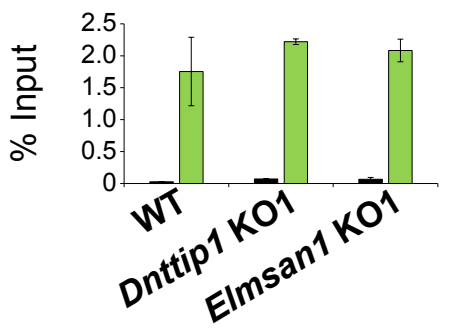

Promoter: p1
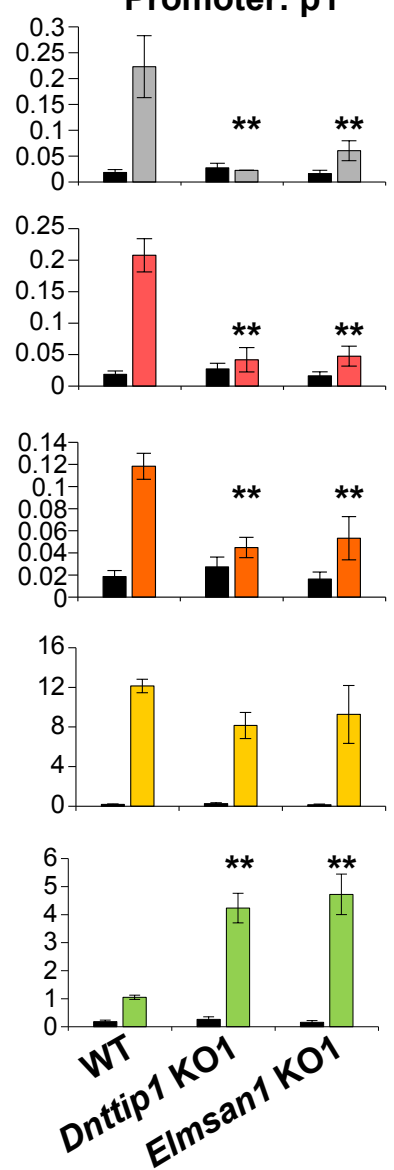

Promoter: p2
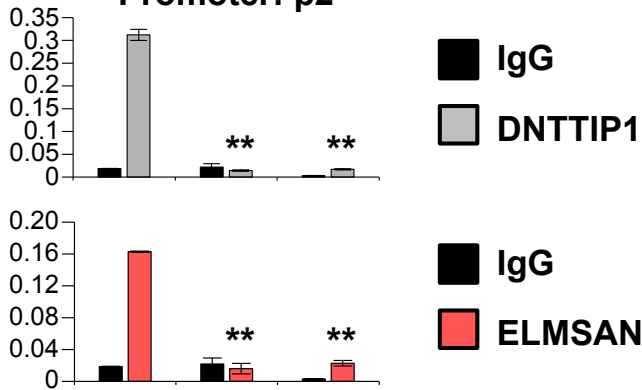

$\lg G$

ELMSAN1

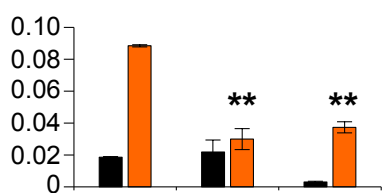

$\lg \mathbf{G}$

HDAC1

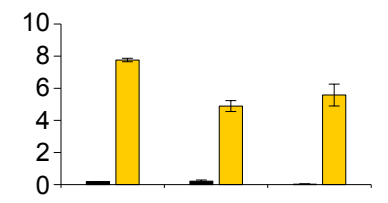

$\lg G$

H3K27ac

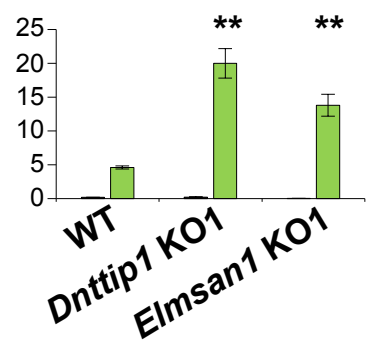

$\lg \mathbf{G}$

H4K20ac

\section{Regulatory regions of the Id1 locus}
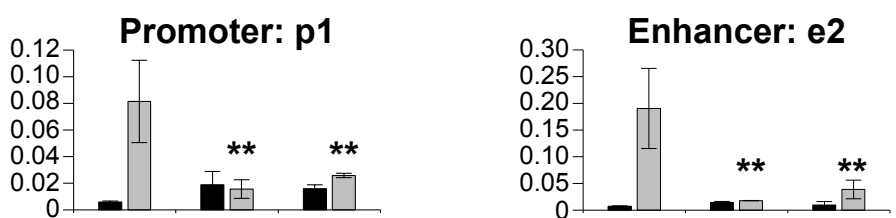

IgG

DNTTIP1
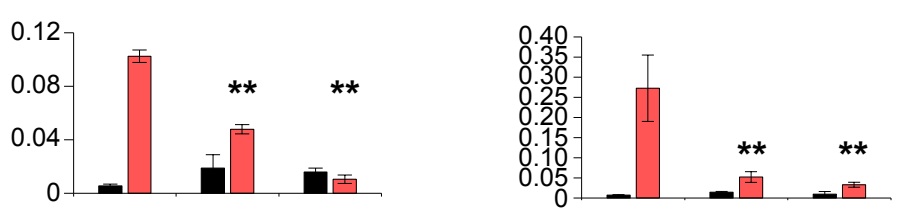

IgG
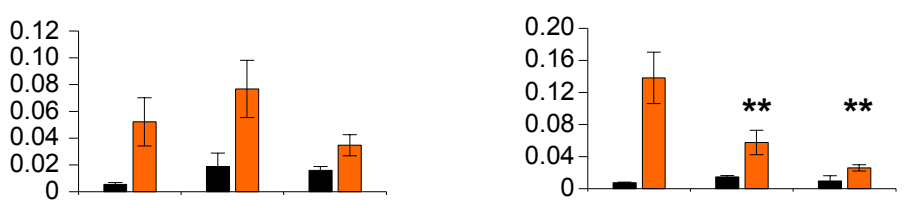

IgG

HDAC1
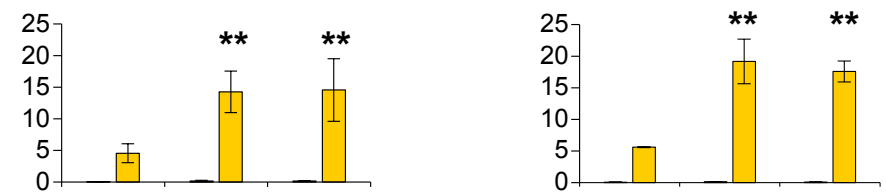

IgG

H3K27ac
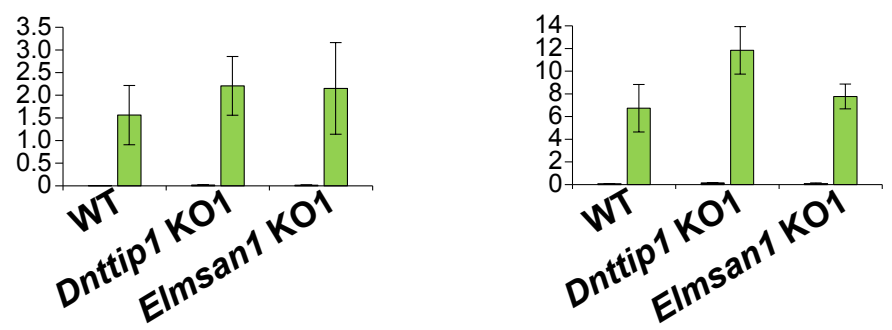

IgG 


\section{Figure S7}

A

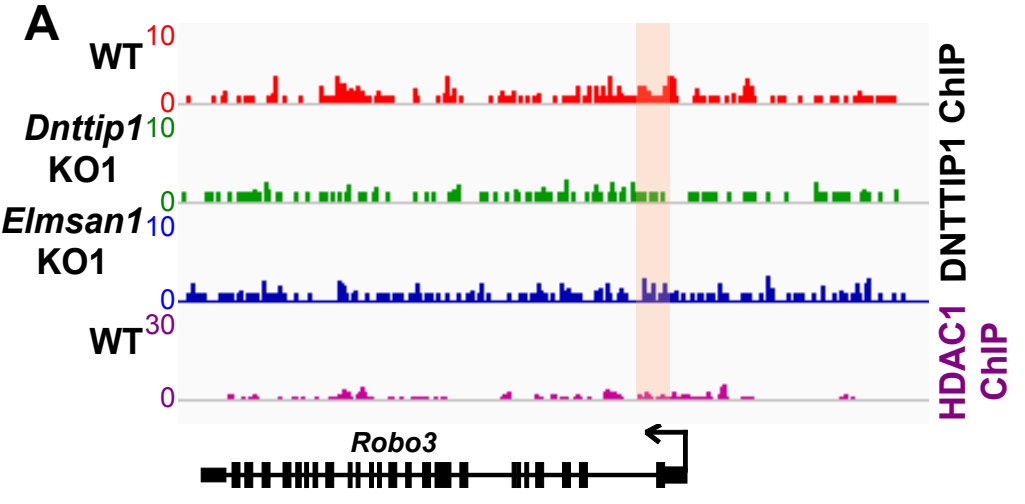

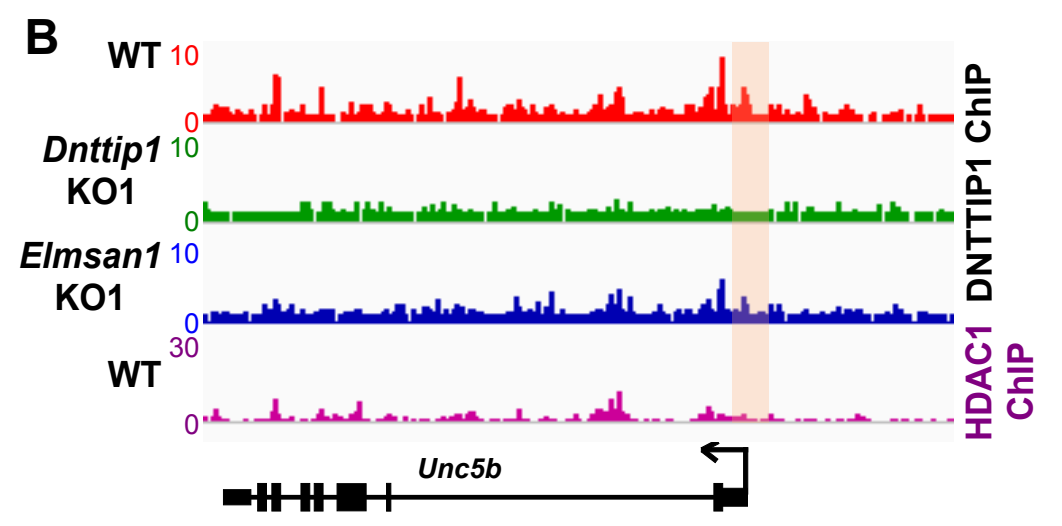

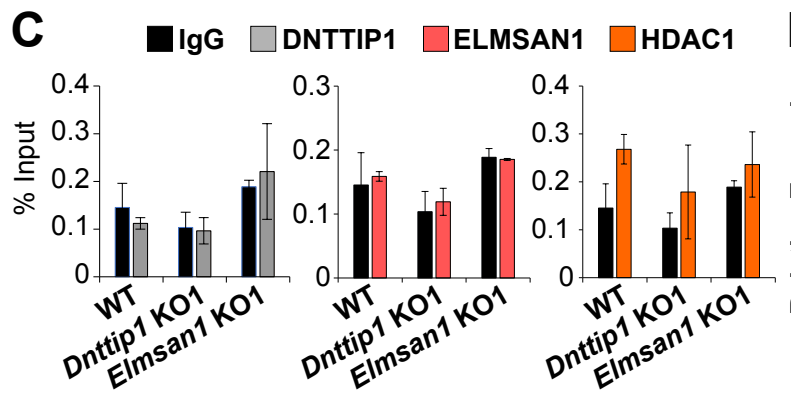

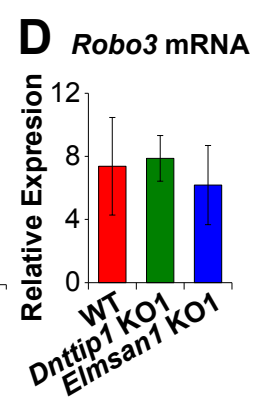

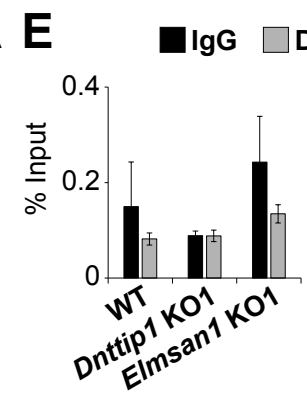

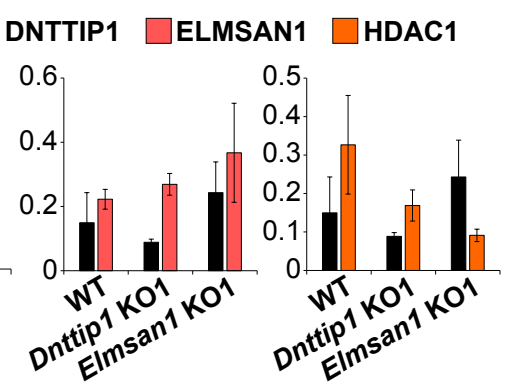

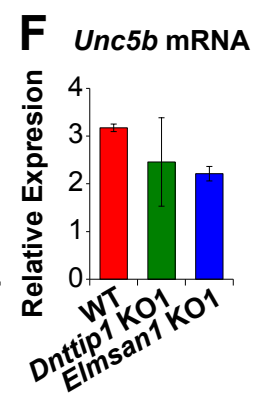




\section{Figure S8}

A

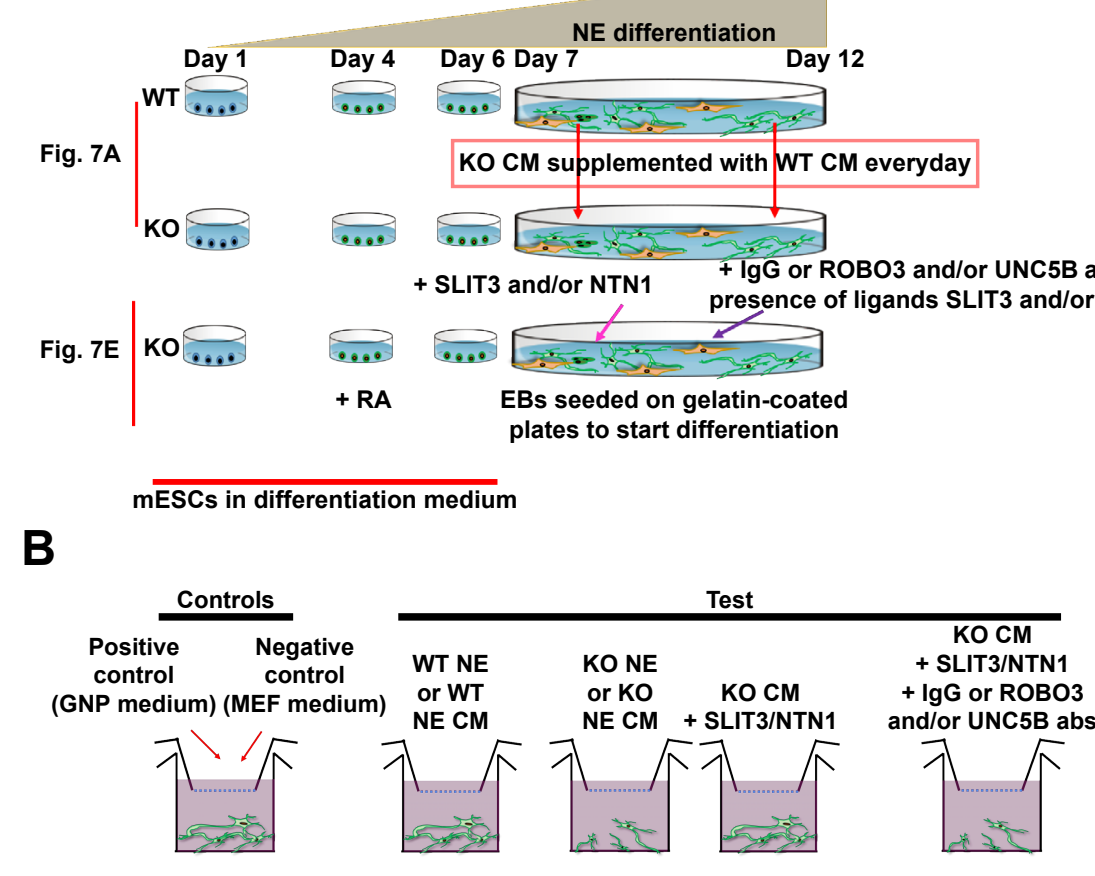

C

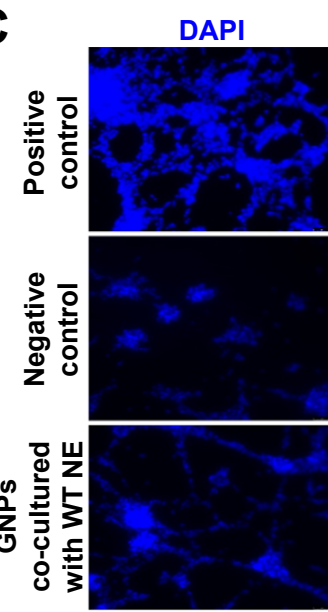

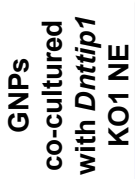

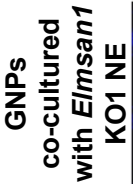

E

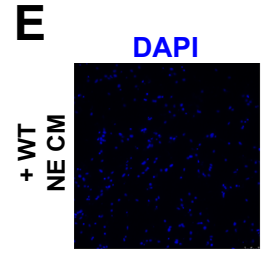

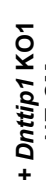

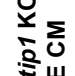

的

岂

$+$

就立

空

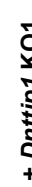

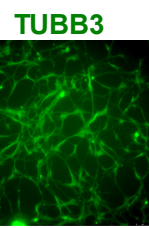
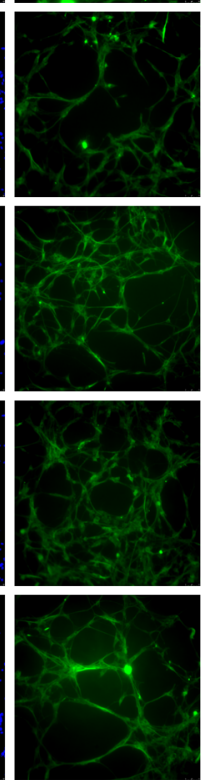
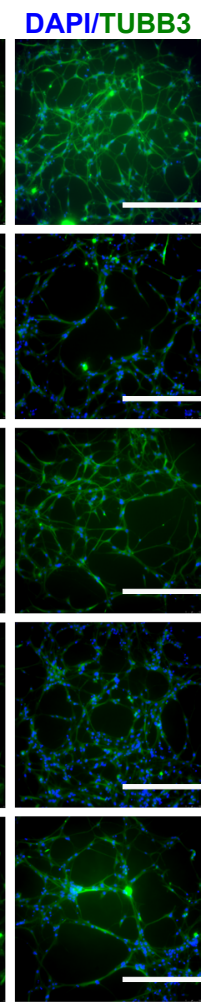
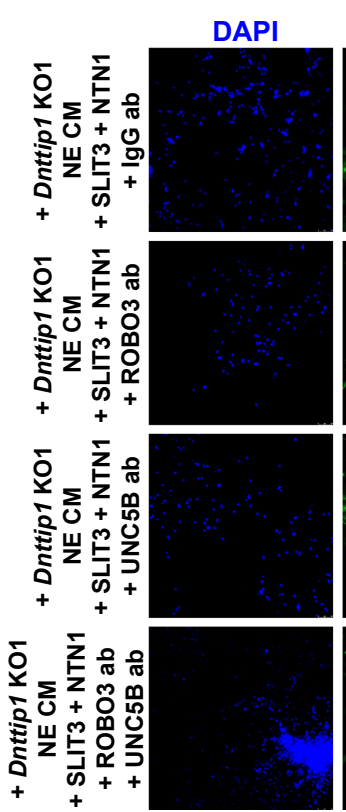

TUBB3
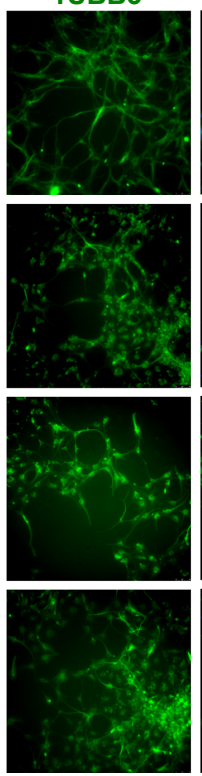

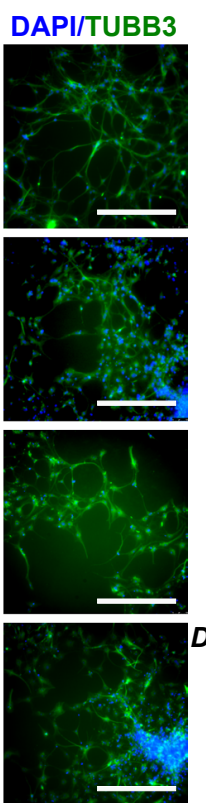

TUBB3

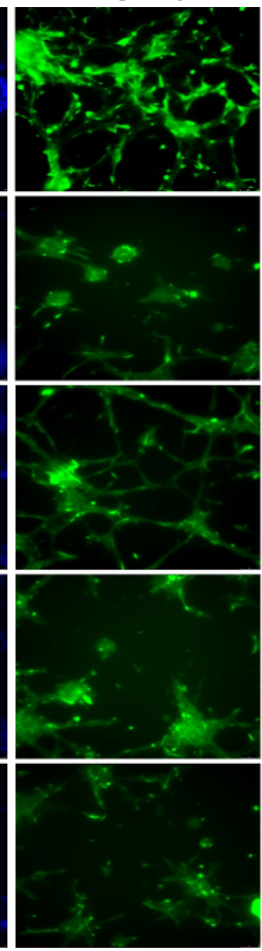

D
DAPI/TUBB3

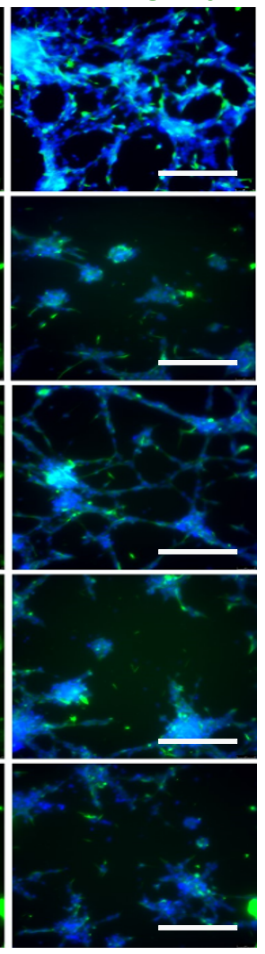

$* * *$

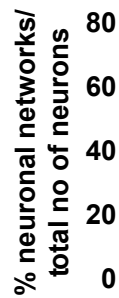

F

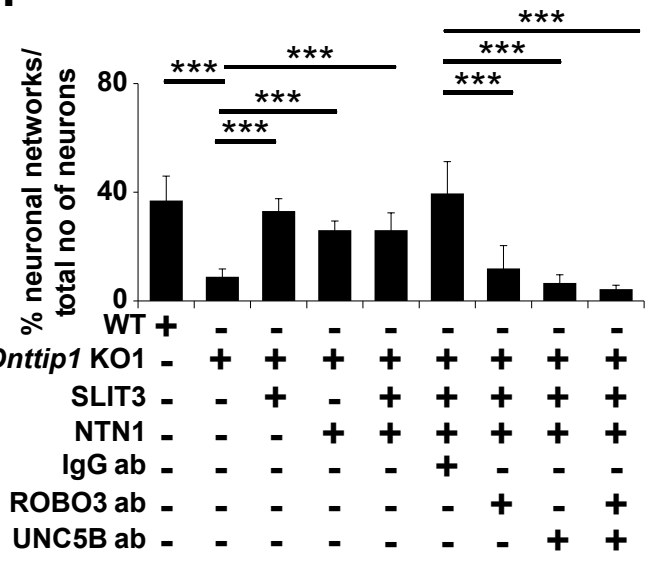


Table S1. List of guide RNA and deep sequencing primer sequences used for CRISPR/Cas9mediated genome editing of Dnttip1 and Elmsan1 in mESCs.

\begin{tabular}{|c|c|}
\hline gRNAs & Sequence (5' to 3') \\
\hline Dnttipl gRNA & ACAUCGGCAGGUGCAGCGAA \\
\hline Elmsan 1 gRNA & GCUCUCUUGGCGGCUAAGGC \\
\hline Deep Sequencing Primers & Sequence (5' to $\left.3^{\prime}\right)$ \\
\hline $\begin{array}{l}\text { Dnttip1.NGS.F } \\
\text { partial Illumina adaptors (upper case) }\end{array}$ & $\begin{array}{l}\text { CACTCTTTCCCTACACGACGCTCTTCCGATCT } \\
\text { ccaaggtgggagaagtgagaagcagg }\end{array}$ \\
\hline parttip1.NGS.R & $\begin{array}{l}\text { GTGACTGGAGTTCAGACGTGTGCTCTTCCGA } \\
\text { TCTececactectgtgctctttacgggc }\end{array}$ \\
\hline $\begin{array}{l}\text { Elmsan1.NGS.F } \\
\text { partial Illumina adaptors (upper case) }\end{array}$ & $\begin{array}{l}\text { CACTCTTTCCCTACACGACGCTCTTCCGATCT } \\
\text { aaacaggggetcaactcetgaacet }\end{array}$ \\
\hline Elmsan1.NGS.R & $\begin{array}{l}\text { GTGACTGGAGTTCAGACGTGTGCTCTTCCGA } \\
\text { TCTcttgtgcteggtggggtactgctca }\end{array}$ \\
\hline
\end{tabular}


Table S5. List of primer sequences used for qRT-PCR analysis.

\begin{tabular}{|c|c|c|}
\hline Gene & Forward Primer (5' to 3') & Reverse Primer (5' to 3') \\
\hline Dnttip1 & CCCATGAGCTTCCAGGAATA & CGAGATCCCAACACAAAGGT \\
\hline Elmsan 1 & AAAGGATGGCAGTGGTTCTG & CCTCAGTTGGGTCCACAGTT \\
\hline Hdacl & GGAAATCTATCGCCCTCACA & AACAGGCCATCGAATACTGG \\
\hline Hdac2 & CGGAGAAGATTGTCCGGTGT & ATTTCACAGCCCCAGCAACT \\
\hline Oct4 & ATGGCATACTGTGGACCTCA & AGCAGCTTGGCAAACTGTTC \\
\hline Nanog & CTCATCAATGCCTGCAGTTTTTCA & CTCCTCAGGGCCCTTGTCAGC \\
\hline Sox2 & GGTTACCTCTTCCTCCCACTCCAG & TCACATGTGCGACAGGGGCAG \\
\hline Pax6 & TGGTATTCTCTCCCCCTCCT & TAAGGATGTTGAACGGGCAG \\
\hline Nes & TGAGGGTCAGGTGGTTCTG & AGAGCAGGGAGGGACATTC \\
\hline Tubb3 & TCCGAGTACCAGCAGTACCA & GGCTTCCGATTCCTCGTCAT \\
\hline Map2 & CCCCATGGCAAACCGCTAA & AAGGCAAGTAGGAGAGGGAGA \\
\hline Slit3 & AGTTGTCTGCCTTCCGACAG & GCACTCGGAGGGATCTTAGC \\
\hline Ntn1 & CACTGCCACTACTGCAAGGA & TAGCCTTTGGCACATCGGTT \\
\hline Ncam1 & AGAGGACGGGAACTCCATCA & GGTTTCCACTCAGAGGCGAG \\
\hline Robo3 & GAATCGCCGAGAGGAACCAA & CACATCGGTTGACCAGGGAA \\
\hline Unc5b & GTGCTGACCCTGGAGACATC & GAGAACCGCTACCACCACAA \\
\hline Spry4 & TTCGGGGATTTACACAGACG & CTGCTGTCAAGGAGGGGC \\
\hline IdI & GAGGCGGCATGTGTTCCA & GAAGGGCTGGAGTCCATCTG \\
\hline Pacsin1 & GCAGGACCACTTCTCTCACC & GCTTGTAGTTCCCCACCTCC \\
\hline Gapdh & TGTGTCCGTCGTGGATCTGA & CCTGCTTCACCACCTTCTTGA \\
\hline
\end{tabular}


Table S6. List of primer sequences used for ChIP qPCR analysis.

\begin{tabular}{|c|c|c|c|}
\hline Gene & $\begin{array}{l}\text { Target } \\
\text { Type }\end{array}$ & Forward Primer (5' to 3') & Reverse Primer (5' to 3') \\
\hline Slit3 & $\begin{array}{l}\text { Promoter } \\
\text { (p1) }\end{array}$ & $\begin{array}{l}\text { CACTGTGGTGAGGAGAG } \\
\text { CTG }\end{array}$ & $\begin{array}{l}\text { CTGAACACCCATCCAGCC } \\
\text { AT }\end{array}$ \\
\hline Slit3 & $\begin{array}{l}\text { Enhancer } 1 \\
\text { (e1) }\end{array}$ & $\begin{array}{l}\text { CCTGCACTCCCTCACTCT } \\
\text { TC }\end{array}$ & $\begin{array}{l}\text { GCTGAACAATCATCCCAG } \\
\text { GT }\end{array}$ \\
\hline Slit3 & $\begin{array}{l}\text { Enhancer } 2 \\
(\mathrm{e} 2)\end{array}$ & $\begin{array}{l}\text { TAAGCTTGCTTGGTGCCT } \\
\text { TT }\end{array}$ & $\begin{array}{l}\text { TGTTGCCCTTGGTAACCTT } \\
\text { C }\end{array}$ \\
\hline Slit3 & $\begin{array}{l}\text { Intragenic } \\
\text { Control } \\
\text { Region } \\
(\mathrm{NC})\end{array}$ & $\begin{array}{l}\text { TCTTGTTCAGAATGCATT } \\
\text { TACAGAT }\end{array}$ & $\begin{array}{l}\text { TCTGTATGTCTCTCAGCA } \\
\text { ACCT }\end{array}$ \\
\hline Ntn1 & $\begin{array}{l}\text { Promoter } 1 \\
(\mathrm{p} 1)\end{array}$ & $\begin{array}{l}\text { TCAAACTGGGGGCTATG } \\
\text { AAG }\end{array}$ & $\begin{array}{l}\text { AGCTCCTGGCCAAACACT } \\
\text { AA }\end{array}$ \\
\hline Ntn1 & $\begin{array}{l}\text { Promoter } 2 \\
\text { (p2) }\end{array}$ & $\begin{array}{l}\text { TATGCCCATTCTCCTTGC } \\
\text { TC }\end{array}$ & $\begin{array}{l}\text { GGCCAGGCAAACTTTTCT } \\
\text { TT }\end{array}$ \\
\hline Ntn1 & $\begin{array}{l}\text { Enhancer } \\
(\mathrm{e} 1)\end{array}$ & $\begin{array}{l}\text { TTTGAGGCAGGGTCTCA } \\
\text { GTT }\end{array}$ & $\begin{array}{l}\text { TGAGGGAGGTGGAGTCA } \\
\text { AAC }\end{array}$ \\
\hline Spry4 & $\begin{array}{l}\text { Promoter } \\
\text { (p1) }\end{array}$ & $\begin{array}{l}\text { TCAGAAATTCGCTTGCCT } \\
\text { CT }\end{array}$ & $\begin{array}{l}\text { TGGCCAACGCTATTTAAA } \\
\text { CC }\end{array}$ \\
\hline Spry4 & $\begin{array}{l}\text { Enhancer } 1 \\
(\mathrm{e} 1)\end{array}$ & $\begin{array}{l}\text { ACGCATTGTTGCTTCTGT } \\
\text { TG }\end{array}$ & $\begin{array}{l}\text { TCATGGCTTGGGACCATA } \\
\text { TT }\end{array}$ \\
\hline Spry4 & $\begin{array}{l}\text { Enhancer } 2 \\
\text { (e2) }\end{array}$ & $\begin{array}{l}\text { GGAGGAAGGAGAAAGG } \\
\text { GATG }\end{array}$ & $\begin{array}{l}\text { CCACCAATCACCACAGAC } \\
\text { AG }\end{array}$ \\
\hline Spry4 & $\begin{array}{l}\text { Intragenic } \\
\text { Control } \\
\text { Region } \\
(\mathrm{NC})\end{array}$ & $\begin{array}{l}\text { TCACCAGCAGGGCAATT } \\
\text { TTATG }\end{array}$ & $\begin{array}{l}\text { AGGAGCCAAAAATCAGC } \\
\text { ACCA }\end{array}$ \\
\hline$I d 1$ & $\begin{array}{l}\text { Promoter } \\
\text { (p1) }\end{array}$ & $\begin{array}{l}\text { CACCTGGGGTCAGAACA } \\
\text { TCT }\end{array}$ & $\begin{array}{l}\text { CTGTTGGAAGATTGGGCT } \\
\text { GT }\end{array}$ \\
\hline$I d 1$ & $\begin{array}{l}\text { Enhancer } 1 \\
(\mathrm{e} 1)\end{array}$ & $\begin{array}{l}\text { CTGGGAATGTAGGCACC } \\
\text { TGT }\end{array}$ & $\begin{array}{l}\text { CTTAGCTGTTGCCCTGGA } \\
\text { AG }\end{array}$ \\
\hline$I d 1$ & $\begin{array}{l}\text { Enhancer } 2 \\
(\mathrm{e} 2)\end{array}$ & $\begin{array}{l}\text { TCCTGCAGCATGTAATCG } \\
\text { AC }\end{array}$ & $\begin{array}{l}\text { ATACTCTGCCTCGGACCT } \\
\text { CA }\end{array}$ \\
\hline Robo3 & Promoter & $\begin{array}{l}\text { GGGCTAAGGACAATACT } \\
\text { GTAGGA }\end{array}$ & $\begin{array}{l}\text { GGCTCATCAACCCTGGAA } \\
\text { TGT }\end{array}$ \\
\hline$U n c 5 b$ & Promoter & $\begin{array}{l}\text { CTCATTTCTCAAATGGGG } \\
\text { CAGT }\end{array}$ & $\begin{array}{l}\text { GCCAGCACTGGGATGACT } \\
\text { TA }\end{array}$ \\
\hline
\end{tabular}

